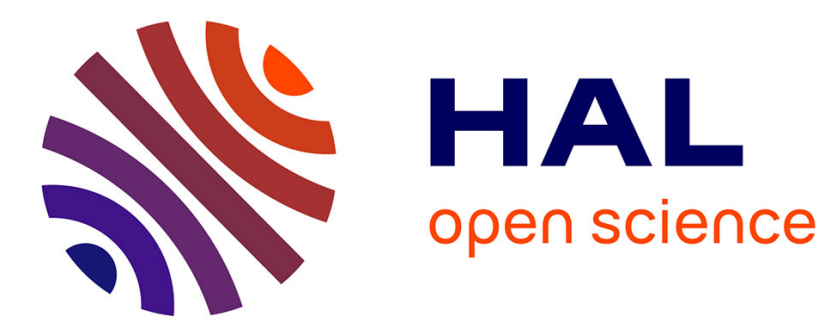

\title{
Local stabilization of a fluid-structure system around a stationary state with a structure given by a finite number of parameters
}

Guillaume Delay

\section{- To cite this version:}

Guillaume Delay. Local stabilization of a fluid-structure system around a stationary state with a structure given by a finite number of parameters. SIAM Journal on Control and Optimization, 2019, 10.1137/18M1177767 . hal-01859852v2

\section{HAL Id: hal-01859852 \\ https://hal.science/hal-01859852v2}

Submitted on 6 Mar 2020

HAL is a multi-disciplinary open access archive for the deposit and dissemination of scientific research documents, whether they are published or not. The documents may come from teaching and research institutions in France or abroad, or from public or private research centers.
L'archive ouverte pluridisciplinaire HAL, est destinée au dépôt et à la diffusion de documents scientifiques de niveau recherche, publiés ou non, émanant des établissements d'enseignement et de recherche français ou étrangers, des laboratoires publics ou privés. 


\title{
Local stabilization of a fluid-structure system around a stationary state with a structure given by a finite number of parameters*
}

\author{
Guillaume Delay ${ }^{\dagger}$
}

September 5, 2019

\begin{abstract}
We study the stabilization of solutions to a $2 \mathrm{~d}$ fluid-structure system by a feedback control law acting on the acceleration of the structure. The structure is described by a finite number of parameters. The modelling of this system and the existence of strong solutions have been previously studied in [11]. We consider an unstable stationary solution to the problem. We assume a unique continuation property for the eigenvectors of the adjoint system. Under this assumption, the nonlinear feedback control that we propose stabilizes the whole fluid-structure system around the stationary solution at any chosen exponential decay rate for small enough initial perturbations. Our method reposes on the analysis of the linearized system and the feedback operator is given by a Riccati equation of small dimension.
\end{abstract}

MSC numbers. 35Q30, 74F10, 76D55, 93D15

\section{Introduction}

The goal of this study is to stabilize a $2 \mathrm{~d}$ fluid-structure interaction problem. The fluid is modelled by the incompressible Navier-Stokes equations and the structure, immersed in the fluid, is governed by two scalar parameters denoted $\theta_{1}$ and $\theta_{2}$. More precisely, the structure is a smooth approximation of a steering gear depending on two angles of deformation $\theta_{1}$ and $\theta_{2}$. Such a kind of structure can be found for instance in aeronautics [18]. Our goal is to design a finite dimensional feedback controller which stabilizes locally the system around a given stationary state at any prescribed exponential decay rate.

Even if we considered only two parameters, the present study can easily be extended to the case of a structure depending on a finite number $N(\geq 1)$ of scalar parameters (see Remark 1.1). The extension of all proofs is indeed straightforward.

\subsection{Modelling of the problem}

The fluid-structure configuration considered in this paper has already been investigated in [11] where existence of strong solutions has been proven. We consider a bounded domain $\Omega=(0, L) \times(0,1)$ (see Fig.1). The volume occupied by the structure depends on two parameters denoted $\left(\theta_{1}, \theta_{2}\right)$, it is a closed subset of $\Omega$ that we denote $S\left(\theta_{1}, \theta_{2}\right) \subset \Omega$. The volume filled by the fluid is denoted $\mathscr{F}\left(\theta_{1}, \theta_{2}\right)=\Omega \backslash S\left(\theta_{1}, \theta_{2}\right)$.

The boundary $\partial \Omega$ can be decomposed into $\partial \Omega=\overline{\Gamma_{\mathrm{i}}} \cup \overline{\Gamma_{\mathrm{w}}} \cup \overline{\Gamma_{\mathrm{N}}}$, where $\Gamma_{\mathrm{i}}=\{0\} \times(0,1), \Gamma_{\mathrm{w}}=(0, L) \times\{0,1\}$ and $\Gamma_{\mathrm{N}}=\{L\} \times(0,1)$. We also denote $\Gamma_{\mathrm{D}}=\overline{\Gamma_{\mathrm{i}}} \cup \Gamma_{\mathrm{w}}$ the part of $\partial \Omega$ where Dirichlet conditions are imposed. We now introduce the equations modelling this system.

\subsubsection{The equations of the fluid}

The velocity of the fluid is assumed to fulfil the incompressible Navier-Stokes equations

$$
\left\{\begin{array}{lll}
\frac{\partial \mathbf{u}}{\partial t}(t, \mathbf{x})+(\mathbf{u}(t, \mathbf{x}) \cdot \nabla) \mathbf{u}(t, \mathbf{x})-\operatorname{div} \sigma_{F}(\mathbf{u}(t, \mathbf{x}), p(t, \mathbf{x}))=\mathbf{f}_{\mathscr{F}}(t, \mathbf{x}), & t \in(0, \infty), & \mathbf{x} \in \mathscr{F}\left(\theta_{1}(t), \theta_{2}(t)\right), \\
\operatorname{div} \mathbf{u}(t, \mathbf{x})=0, & t \in(0, \infty), & \mathbf{x} \in \mathscr{F}\left(\theta_{1}(t), \theta_{2}(t)\right), \\
\mathbf{u}(t, \mathbf{x})=\mathbf{u}^{i}(t, \mathbf{x}), & t \in(0, \infty), & \mathbf{x} \in \Gamma_{\mathrm{i}}, \\
\mathbf{u}(t, \mathbf{x})=0, & t \in(0, \infty), & \mathbf{x} \in \Gamma_{\mathrm{w}}, \\
\sigma_{F}(\mathbf{u}(t, \mathbf{x}), p(t, \mathbf{x})) \mathbf{n}(\mathbf{x})=0, & t \in(0, \infty), & \mathbf{x} \in \Gamma_{\mathrm{N}}, \\
\mathbf{u}(t, \mathbf{x})=\mathbf{v}_{s}(t, \mathbf{x}), & t \in(0, \infty), & \mathbf{x} \in \partial S\left(\theta_{1}(t), \theta_{2}(t)\right), \\
\mathbf{u}(0, \mathbf{x})=\mathbf{u}_{0}(\mathbf{x}), & & \mathbf{x} \in \mathscr{F}\left(\theta_{1,0}, \theta_{2,0}\right),
\end{array}\right.
$$

where $\mathbf{u}(t, \mathbf{x})$ and $p(t, \mathbf{x})$ are the velocity and the pressure of the fluid at point $\mathbf{x}$ and time $t$,

$$
\sigma_{F}(\mathbf{u}, p)=\nu\left(\nabla \mathbf{u}+(\nabla \mathbf{u})^{T}\right)-p \mathbf{I},
$$

\footnotetext{
* The author is partially supported by IFSMACS ANR-15-CE40-0010

$\dagger$ Institut de Mathématiques de Toulouse, Université Paul Sabatier - 118, route de Narbonne F-31062 Toulouse Cedex 9, France (guillaume.delay@math.univ-toulouse.fr or guillaume.delay@enpc.fr)
} 


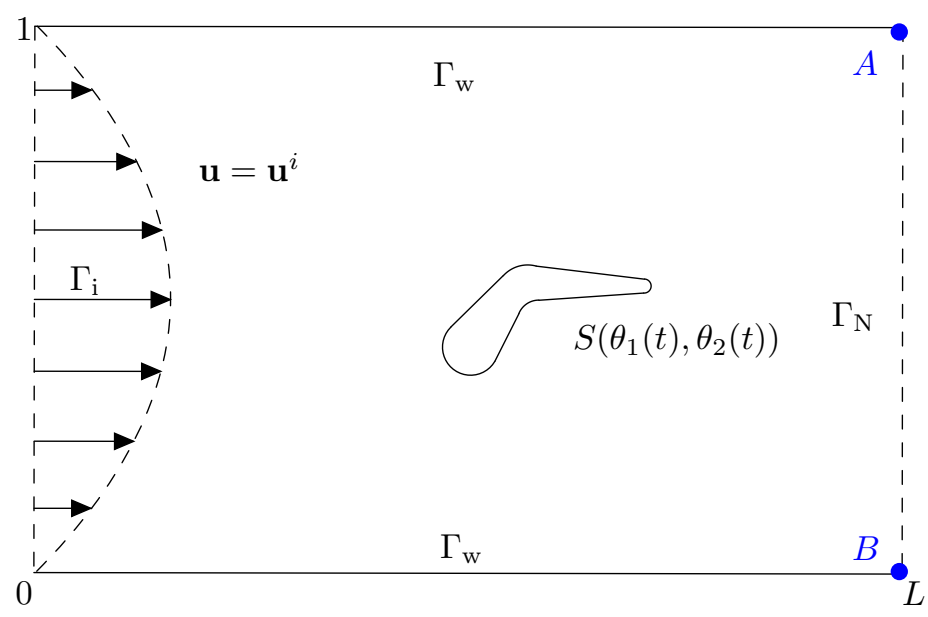

Figure 1: The geometrical configuration.

is the Cauchy stress tensor of the fluid and $\nu>0$ is the kinematic viscosity. The term $\mathbf{f}_{\mathscr{F}}(t, \mathbf{x})$ in $(1.1)_{1}$ is a force per unit mass exerted on the fluid, $\mathbf{u}^{i}(t, \mathbf{x})$ is a nonhomogeneous boundary datum on $\Gamma_{\mathrm{i}}, \mathbf{v}_{s}(t, \mathbf{x})$ denotes the velocity of the structure and $\mathbf{n}(\mathbf{x})$ is the outward unit normal to $\Omega$. Dirichlet boundary conditions are imposed on $\Gamma_{\mathrm{D}}$ and Neumann type (free output) boundary conditions are imposed on $\Gamma_{\mathrm{N}}$. We also consider an initial datum $\mathbf{u}_{0}(\mathbf{x})$ for the fluid velocity.

\subsubsection{Equations of the structure}

We consider that the couple of parameters $\left(\theta_{1}, \theta_{2}\right)$ lies in an admissible domain $\mathbb{D}_{\Theta}$ which is an open connected subset of $\mathbb{R}^{2}$ containing $(0,0)$. These parameters stand here for structure deformation angles (see Fig. 2).

We consider a function $\mathbf{X}$ defined on $\mathrm{D}_{\Theta} \times S(0,0)$ that computes the position of a point of the structure according to its reference position in $S(0,0)$ and the value of the parameters $\left(\theta_{1}, \theta_{2}\right) \in \mathbb{D}_{\Theta}$.

Let us list below the assumptions that we make

\section{Modelling Assumptions.}

- For every $\mathbf{y} \in S(0,0), \mathbf{X}(0,0, \mathbf{y})=\mathbf{y}$.

- The set $S(0,0)$ is a smooth simply connected closed subset of $\Omega$.

- For every $\left(\theta_{1}, \theta_{2}\right) \in \mathbb{D}_{\Theta}, \mathbf{X}\left(\theta_{1}, \theta_{2}, S(0,0)\right)=S\left(\theta_{1}, \theta_{2}\right) \subset \Omega$ and $\inf _{\left(\theta_{1}, \theta_{2}\right) \in \mathbb{D}_{\Theta}} \mathrm{d}\left(S\left(\theta_{1}, \theta_{2}\right), \partial \Omega\right)>0$.

- For every $\left(\theta_{1}, \theta_{2}\right) \in \mathbb{D}_{\Theta}, \mathbf{X}\left(\theta_{1}, \theta_{2},.\right)$ is a $\mathscr{C}^{\infty}$ diffeomorphism from $S(0,0)$ to its image $S\left(\theta_{1}, \theta_{2}\right)$.

- The function $\mathbf{X}$ is $\mathscr{C}^{\infty}$ on $\mathbb{D}_{\Theta} \times S(0,0)$.

- The functions $\partial_{\theta_{1}} \mathbf{X}\left(\theta_{1}, \theta_{2},.\right)$ and $\partial_{\theta_{2}} \mathbf{X}\left(\theta_{1}, \theta_{2},.\right)$ form

$$
\text { a free family in } \mathbf{L}^{2}(\partial S(0,0)) \text { for every }\left(\theta_{1}, \theta_{2}\right) \text { in } \mathbb{D}_{\Theta} \text {. }
$$

- No friction and no elastic energy are considered in the structure.

More information about these assumptions can be found in [11]. The inverse diffeomorphism of $\mathbf{X}\left(\theta_{1}, \theta_{2},.\right)$, whose existence is guaranteed by $(1.5)$, is denoted $\mathbf{Y}\left(\theta_{1}, \theta_{2},.\right)$ and we have

$$
\forall\left(\theta_{1}, \theta_{2}\right) \in \mathbb{D}_{\Theta}, \quad \forall \mathbf{y} \in S(0,0), \quad \mathbf{Y}\left(\theta_{1}, \theta_{2}, \mathbf{X}\left(\theta_{1}, \theta_{2}, \mathbf{y}\right)\right)=\mathbf{y} .
$$

The diffeomorphisms $\mathbf{X}\left(\theta_{1}, \theta_{2},.\right)$ and $\mathbf{Y}\left(\theta_{1}, \theta_{2},.\right)$ are illustrated in Fig. 2.

In the sequel, we denote $\dot{\theta}_{j}$ and $\ddot{\theta}_{j}$ the first and second time derivatives of $\theta_{j}$. The equations that are satisfied by the structure read on a matrix form

$$
\mathcal{M}_{\theta_{1}, \theta_{2}}\left(\begin{array}{c}
\ddot{\theta}_{1} \\
\ddot{\theta}_{2}
\end{array}\right)=\mathbf{M}_{\mathbf{I}}\left(\theta_{1}, \theta_{2}, \dot{\theta}_{1}, \dot{\theta}_{2}\right)+\mathbf{M}_{\mathbf{A}}\left(\theta_{1}, \theta_{2},-\sigma_{F}(\mathbf{u}, p) \mathbf{n}_{\theta_{1}, \theta_{2}}\right)+\mathbf{f}_{\mathbf{s}}+\mathbf{h} \quad \text { on }(0, T),
$$

where $\mathbf{f}_{\mathbf{s}}$ is a source term, $\mathbf{h}$ a control function,

$$
\begin{aligned}
& \mathcal{M}_{\theta_{1}, \theta_{2}}=\left(\begin{array}{cc}
\left(\partial_{\theta_{1}} \mathbf{X}\left(\theta_{1}, \theta_{2}, .\right), \partial_{\theta_{1}} \mathbf{X}\left(\theta_{1}, \theta_{2}, .\right)\right)_{S} & \left(\partial_{\theta_{2}} \mathbf{X}\left(\theta_{1}, \theta_{2}, .\right), \partial_{\theta_{1}} \mathbf{X}\left(\theta_{1}, \theta_{2}, .\right)\right)_{S} \\
\left(\partial_{\theta_{1}} \mathbf{X}\left(\theta_{1}, \theta_{2}, .\right), \partial_{\theta_{2}} \mathbf{X}\left(\theta_{1}, \theta_{2}, .\right)\right)_{S} & \left(\partial_{\theta_{2}} \mathbf{X}\left(\theta_{1}, \theta_{2}, .\right), \partial_{\theta_{2}} \mathbf{X}\left(\theta_{1}, \theta_{2}, .\right)\right)_{S}
\end{array}\right) \in \mathbb{R}^{2 \times 2}
\end{aligned}
$$

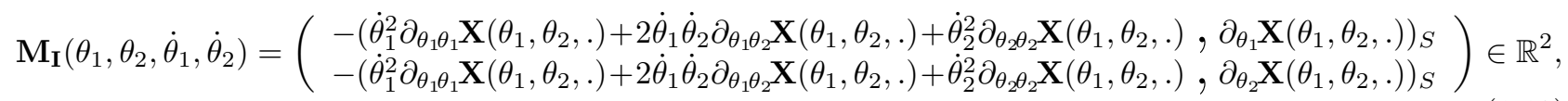

where $(., .)_{S}$ is the scalar product

$$
(\mathbf{\Phi}, \boldsymbol{\Psi})_{S}=\int_{S(0,0)} \rho \mathbf{\Phi}(\mathbf{y}) \cdot \mathbf{\Psi}(\mathbf{y}) \mathrm{d} \mathbf{y}
$$




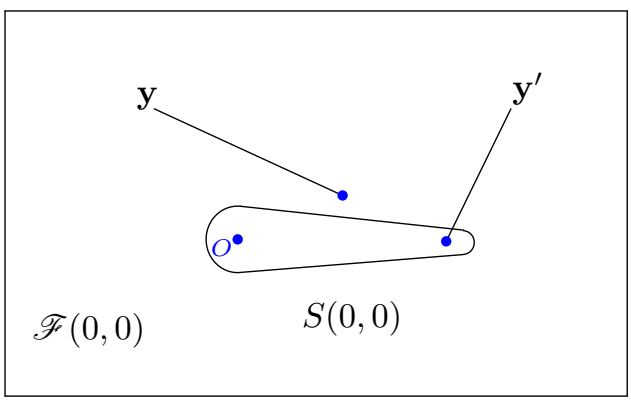

$\Omega$
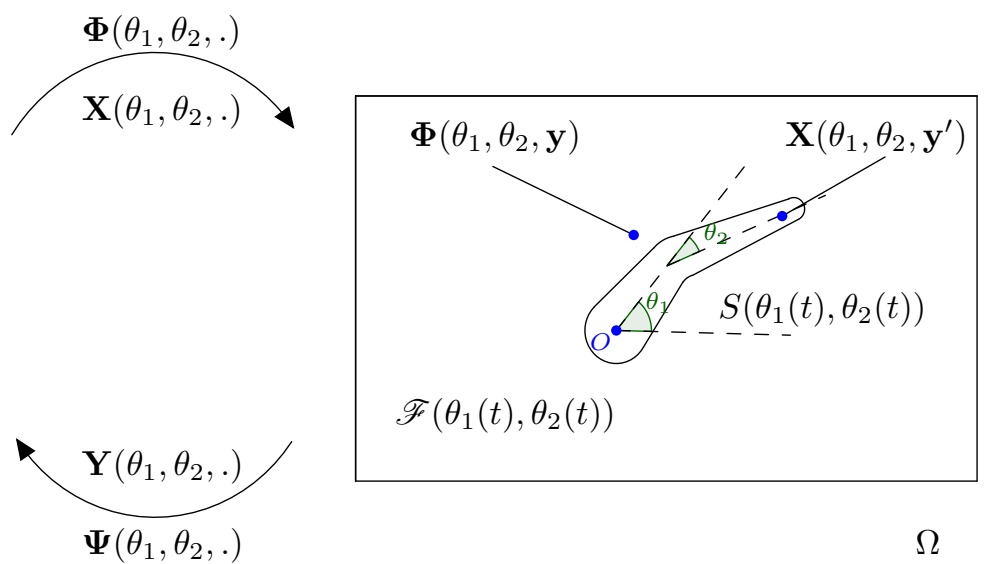

$\Omega$

Figure 2: Correspondence between real and reference configurations.

with $\rho>0$ the mass per unit volume of the structure and

$$
\mathbf{M}_{\mathbf{A}}\left(\theta_{1}, \theta_{2},-\sigma_{F}(\mathbf{u}, p) \mathbf{n}_{\theta_{1}, \theta_{2}}\right)=\left(\begin{array}{l}
\int_{\partial S\left(\theta_{1}, \theta_{2}\right)}-\sigma_{F}(\mathbf{u}, p) \mathbf{n}_{\theta_{1}, \theta_{2}}\left(\gamma_{x}\right) \cdot \partial_{\theta_{1}} \mathbf{X}\left(\theta_{1}, \theta_{2}, \mathbf{Y}\left(\theta_{1}, \theta_{2}, \gamma_{x}\right)\right) \mathrm{d} \gamma_{x} \\
\int_{\partial S\left(\theta_{1}, \theta_{2}\right)}-\sigma_{F}(\mathbf{u}, p) \mathbf{n}_{\theta_{1}, \theta_{2}}\left(\gamma_{x}\right) \cdot \partial_{\theta_{2}} \mathbf{X}\left(\theta_{1}, \theta_{2}, \mathbf{Y}\left(\theta_{1}, \theta_{2}, \gamma_{x}\right)\right) \mathrm{d} \gamma_{x}
\end{array}\right) \in \mathbb{R}^{2},
$$

where $\mathbf{n}_{\theta_{1}, \theta_{2}}$ is the outward unit normal to $\mathscr{F}\left(\theta_{1}, \theta_{2}\right)$ on $\partial S\left(\theta_{1}, \theta_{2}\right)$.

Moreover the velocity of the structure can be written

$$
\forall t \in[0, \infty), \quad \forall x \in S\left(\theta_{1}(t), \theta_{2}(t)\right), \quad \mathbf{v}_{s}(t, \mathbf{x})=\sum_{k=1}^{2} \dot{\theta}_{k}(t) \partial_{\theta_{k}} \mathbf{X}\left(\theta_{1}(t), \theta_{2}(t), \mathbf{Y}\left(\theta_{1}(t), \theta_{2}(t), \mathbf{x}\right)\right) .
$$

More information about the derivation of these equations can be found in [11].

Note that the matrix $\mathcal{M}_{\theta_{1}, \theta_{2}}$ in (1.11) is the Gram matrix of the family $\left(\partial_{\theta_{1}} \mathbf{X}\left(\theta_{1}, \theta_{2}\right), \partial_{\theta_{2}} \mathbf{X}\left(\theta_{1}, \theta_{2}\right)\right)$ with respect to the scalar product $(., .)_{S}$. It is thus invertible due to Assumption (1.7) (if two $\mathscr{C}^{\infty}$ functions are not collinear in $\mathbf{L}^{2}(\partial S(0,0))$ then they are not collinear in $\left.\mathbf{L}^{2}(S(0,0))\right)$.

Remark 1.1. The proposed framework can be used to model other problems. For instance, in the case of a rigid solid whose center of mass is given by $\left(a_{1}, a_{2}\right)$ and corresponds to $(0,0)$ in the reference configuration and whose angle of rotation is given by $\theta$ (so that three parameters are considered), the diffeomorphism $\mathbf{X}$ now depends on three parameters and is given by

$$
\mathbf{X}\left(a_{1}, a_{2}, \theta, \mathbf{y}\right)=a_{1} \mathbf{e}_{1}+a_{2} \mathbf{e}_{2}+R_{\theta} \mathbf{y},
$$

where $\mathbf{e}_{1}=(1,0), \mathbf{e}_{2}=(0,1)$ and $R_{\theta}=\left(\begin{array}{cc}\cos (\theta) & -\sin (\theta) \\ \sin (\theta) & \cos (\theta)\end{array}\right)$. Moreover, we have

$\mathcal{M}_{a_{1}, a_{2}, \theta}=\left(\begin{array}{ccc}m & 0 & 0 \\ 0 & m & 0 \\ 0 & 0 & I\end{array}\right), \quad \mathbf{M}_{\mathbf{I}}\left(a_{1}, a_{2}, \theta, \dot{a}_{1}, \dot{a}_{2}, \dot{\theta}\right)=0, \quad \mathbf{M}_{\mathbf{A}}\left(a_{1}, a_{2}, \theta, \mathbf{f}\right)=\int_{\partial S\left(a_{1}, a_{2}, \theta\right)}\left(\begin{array}{l}\mathbf{f} \cdot \mathbf{e}_{1} \\ \mathbf{f} \cdot \mathbf{e}_{2} \\ \mathbf{f} \cdot R_{\theta+\frac{\pi}{2}} \mathbf{y}\end{array}\right) \mathrm{d} \mathbf{x}$,

where $m=\int_{S(0,0,0)} \rho \mathrm{d} \mathbf{y}$ denotes the mass of the solid and $I=\int_{S(0,0,0)} \rho \mathbf{y}^{2} \mathrm{~d} \mathbf{y}$ its moment of inertia. Hence equation (1.10) corresponds to the usual Newton's law. 


\subsubsection{The complete set of equations}

The final system that we consider is given by the following set of equations

$$
\begin{cases}\frac{\partial \mathbf{u}}{\partial t}(t, \mathbf{x})+(\mathbf{u}(t, \mathbf{x}) \cdot \nabla) \mathbf{u}(t, \mathbf{x})-\operatorname{div} \sigma_{F}(\mathbf{u}(t, \mathbf{x}), p(t, \mathbf{x}))=\mathbf{f}_{\mathscr{F}}(t, \mathbf{x}), & t \in(0, \infty), \mathbf{x} \in \mathscr{F}\left(\theta_{1}(t), \theta_{2}(t)\right), \\ \operatorname{div} \mathbf{u}(t, \mathbf{x})=0, & t \in(0, \infty), \mathbf{x} \in \mathscr{F}\left(\theta_{1}(t), \theta_{2}(t)\right), \\ \mathbf{u}(t, \mathbf{x})=\sum_{j=1}^{2} \dot{\theta}_{j}(t) \partial_{\theta_{j}} \mathbf{X}\left(\theta_{1}(t), \theta_{2}(t), \mathbf{Y}\left(\theta_{1}(t), \theta_{2}(t), \mathbf{x}\right)\right), & t \in(0, \infty), \mathbf{x} \in \partial S\left(\theta_{1}(t), \theta_{2}(t)\right), \\ \mathbf{u}(t, \mathbf{x})=\mathbf{u}^{i}(t, \mathbf{x}), & t \in(0, \infty), \mathbf{x} \in \Gamma_{\mathrm{i}}, \\ \mathbf{u}(t, \mathbf{x})=0, & t \in(0, \infty), \mathbf{x} \in \Gamma_{\mathrm{w}}, \\ \sigma_{F}(\mathbf{u}(t, \mathbf{x}), p(t, \mathbf{x})) \mathbf{n}(\mathbf{x})=0, & t \in(0, \infty), \mathbf{x} \in \Gamma_{\mathrm{N}}, \\ \mathbf{u}(0, \mathbf{x})=\mathbf{u}_{0}(\mathbf{x}), & \mathbf{x} \in \mathscr{F}\left(\theta_{1,0}, \theta_{2,0}\right), \\ \mathcal{M}_{\theta_{1}, \theta_{2}}\left(\ddot{\theta}_{1} \ddot{\theta}_{2}\right)=\mathbf{M}_{\mathbf{I}}\left(\theta_{1}, \theta_{2}, \dot{\theta}_{1}, \dot{\theta}_{2}\right)+\mathbf{M}_{\mathbf{A}}\left(\theta_{1}, \theta_{2},-\sigma_{F}(\mathbf{u}, p) \mathbf{n}_{\theta_{1}, \theta_{2}}\right)+\mathbf{f}_{\mathbf{s}}+\mathbf{h}, t \in(0, \infty), & \\ \theta_{1}(0)=\theta_{1,0}, \quad \theta_{2}(0)=\theta_{2,0}, & \\ \dot{\theta}_{1}(0)=\omega_{1,0}, \quad \dot{\theta}_{2}(0)=\omega_{2,0} . & \end{cases}
$$

Note that the fluid domain $\mathscr{F}\left(\theta_{1}(t), \theta_{2}(t)\right)$ changes over time. The control $\mathbf{h}$ can be understood as a force acting on the structure. The data $\left(\theta_{1,0}, \theta_{2,0}\right)$ and $\left(\omega_{1,0}, \omega_{2,0}\right)$ respectively describe the initial position and velocity of the structure.

In the sequel, since we consider the stabilization of these equations around a stationary state, the data $\mathbf{f}_{\mathscr{F}}$, $\mathbf{u}^{i}$ and $\mathbf{f}_{\mathbf{s}}$ will be considered as time independent.

\subsection{Statement of the main result}

Existence of strong solutions to (1.15) locally in time has been proven in [11]. The goal of the present study is to prove that, given a stationary state, we can choose $\mathbf{h}$ under a feedback form such that a solution to (1.15) stabilizes exponentially around that stationary state when $t$ tends to infinity. In this section we present our stabilization result.

The stationary state. Let $\left(\mathbf{w}, p_{\mathbf{w}}, \eta_{1}, \eta_{2}\right)$ be a stationary state of (1.15) associated to stationary source terms $\mathbf{f}_{\mathscr{F}}, \mathbf{f}_{\mathbf{s}}$ and boundary datum $\mathbf{u}^{i}$, i.e.

$$
\begin{cases}(\mathbf{w}(\mathbf{x}) \cdot \nabla) \mathbf{w}(\mathbf{x})-\operatorname{div} \sigma_{F}\left(\mathbf{w}(\mathbf{x}), p_{\mathbf{w}}(\mathbf{x})\right)=\mathbf{f}_{\mathscr{F}}(\mathbf{x}), & \mathbf{x} \in \mathscr{F}\left(\eta_{1}, \eta_{2}\right), \\ \operatorname{div} \mathbf{w}(\mathbf{x})=0, & \mathbf{x} \in \mathscr{F}\left(\eta_{1}, \eta_{2}\right), \\ \mathbf{w}(\mathbf{x})=0, & \mathbf{x} \in \partial S\left(\eta_{1}, \eta_{2}\right), \\ \mathbf{w}(\mathbf{x})=\mathbf{u}^{i}(\mathbf{x}), & \mathbf{x} \in \Gamma_{\mathrm{i}}, \\ \mathbf{w}(\mathbf{x})=0, & \mathbf{x} \in \Gamma_{\mathbf{w}}, \\ \sigma_{F}\left(\mathbf{w}(\mathbf{x}), p_{\mathbf{w}}(\mathbf{x})\right) \mathbf{n}=0, & \mathbf{x} \in \Gamma_{\mathrm{N}}, \\ 0=\mathbf{M}_{\mathbf{I}}\left(\eta_{1}, \eta_{2}, 0,0\right)+\mathbf{M}_{\mathbf{A}}\left(\eta_{1}, \eta_{2},-\sigma_{F}\left(\mathbf{w}, p_{\mathbf{w}}\right) \mathbf{n}_{\eta_{1}, \eta_{2}}\right)+\mathbf{f}_{\mathbf{s}} . & \end{cases}
$$

Note that $\mathbf{M}_{\mathbf{I}}\left(\eta_{1}, \eta_{2}, 0,0\right)=0$ and it can thus be withdrawn from (1.16).

In the sequel, we take $\left(\eta_{1}, \eta_{2}\right)=(0,0)$ to simplify the notations. This choice is not restrictive as a change of variables can bring the stationary parameters to $(0,0)$. We denote respectively $\mathscr{F}_{s}$ and $S_{s}$ the fluid and solid domains associated to the stationary solution,

$$
\mathscr{F}_{s}=\mathscr{F}(0,0) \quad \text { and } \quad S_{s}=S(0,0) .
$$

Rewriting (1.16), we consider nonhomogeneous terms $\mathbf{f}_{\mathscr{F}}, \mathbf{u}^{i}, \mathbf{f}_{\mathbf{s}}$ and a velocity-pressure profile $\left(\mathbf{w}, p_{\mathbf{w}}\right) \in$ $\mathbf{H}^{3 / 2}\left(\mathscr{F}_{s}\right) \times \mathrm{H}^{1 / 2}\left(\mathscr{F}_{s}\right)$ fulfilling the equations

$$
\begin{cases}-\operatorname{div} \sigma_{F}\left(\mathbf{w}, p_{\mathbf{w}}\right)=-(\mathbf{w} \cdot \nabla) \mathbf{w}+\mathbf{f}_{\mathscr{F}} & \text { in } \mathscr{F}_{s}, \\ \operatorname{div} \mathbf{w}=0 & \text { in } \mathscr{F}_{s}, \\ \mathbf{w}=0 & \text { on } \partial S_{s}, \\ \mathbf{w}=\mathbf{u}^{i} & \text { on } \Gamma_{\mathrm{i}}, \\ \mathbf{w}=0 & \text { on } \Gamma_{\mathbf{w}}, \\ \sigma_{F}\left(\mathbf{w}, p_{\mathbf{w}}\right) \mathbf{n}=0 & \text { on } \Gamma_{\mathrm{N}}, \\ \left(f_{s}\right)_{j}=\int_{\partial S_{s}}\left(\sigma_{F}\left(\mathbf{w}, p_{\mathbf{w}}\right) \mathbf{n}_{s}\right)\left(\gamma_{y}\right) \cdot \partial_{\theta_{j}} \mathbf{X}\left(0,0, \gamma_{y}\right) \mathrm{d} \gamma_{y}, & \end{cases}
$$

where $\mathbf{n}_{s}$ is the outward unit normal to $\mathscr{F}_{s}$ on $\partial S_{s}$,

$$
\mathbf{f}_{\mathscr{F}} \in \mathbf{W}^{1, \infty}(\Omega) \quad \text { and } \quad \mathbf{u}^{i} \in \mathbf{U}^{i}=\left\{\begin{array}{ccc}
\mathbf{u}^{i} \in \mathbf{H}^{3 / 2}\left(\Gamma_{\mathrm{i}}\right) \quad \mid \quad \mathbf{u}_{\mid \partial \Gamma_{\mathrm{i}}}^{i}=0, & \int_{0}^{1 / 4} \frac{\left|\partial_{y_{2}} u_{2}^{i}\left(y_{2}\right)\right|^{2}}{y_{2}} \mathrm{~d} y_{2}<+\infty, \\
& \int_{3 / 4}^{1} \frac{\left|\partial_{y_{2}} u_{2}^{i}\left(y_{2}\right)\right|^{2}}{1-y_{2}} \mathrm{~d} y_{2}<+\infty
\end{array}\right\} .
$$


Remark 1.2. The regularity of the source term $\mathbf{f}_{\mathscr{F}} \in \mathbf{W}^{1, \infty}(\Omega)$ is used for the estimation of some nonlinear terms in Appendix C.

Remark 1.3. We do not consider that (1.17) admits a unique solution $\left(\mathbf{w}, p_{\mathbf{w}}\right) \in \mathbf{H}^{3 / 2}\left(\mathscr{F}_{s}\right) \times \mathrm{H}^{1 / 2}\left(\mathscr{F}_{s}\right)$ for every set of regular data $\left(\mathbf{f}_{\mathscr{F}}, \mathbf{u}^{i}, \mathbf{f}_{\mathbf{s}}\right)$ satisfying some compatibility conditions. However, we consider that there exist a stationary solution $\left(\mathbf{w}, p_{\mathbf{w}}\right) \in \mathbf{H}^{3 / 2}\left(\mathscr{F}_{s}\right) \times \mathrm{H}^{1 / 2}\left(\mathscr{F}_{s}\right)$ and some associated data $\left(\mathbf{f}_{\mathscr{F}}, \mathbf{u}^{i}, \mathbf{f}_{\mathbf{s}}\right)$ such that $(1.17)$ is fulfilled. More information about stationary solutions can be found in [23, Appendix].

The diffeomorphism $\mathbf{\Phi}$. A classical difficulty in fluid-structure problems is that the fluid domain changes over time. The classical way of getting rid of this difficulty is to use a change of variables on $\mathbf{u}$ and $p$ in order to bring the study back into a fixed domain. This procedure uses a diffeomorphism that we have to define properly.

When the state of the structure depends only on a finite number of parameters, it is convenient to construct this diffeomorphism as an extension of the structure deformation into the fluid domain. The diffeomorphism used is defined as an extension of the diffeomorphism $\mathbf{X}$ given for the structure. For that reason, we use the following extension operator.

Lemma 1.4. There exists a linear extension operator $\mathcal{E}: \mathbf{W}^{3, \infty}\left(S_{s}\right) \rightarrow \mathbf{W}^{3, \infty}(\Omega) \cap \mathbf{H}_{0}^{1}(\Omega)$ such that for every $\varphi \in \mathbf{W}^{3, \infty}\left(S_{s}\right)$,

(i) $\mathcal{E}(\varphi)=\varphi$ in $S_{s}$,

(ii) $\mathcal{E}(\varphi)$ has support within $\Omega_{\varepsilon}=\{\mathbf{x} \in \Omega \mid \mathrm{d}(x, \partial \Omega)>\varepsilon\}$ for some $\varepsilon>0$

such that $\mathrm{d}\left(S\left(\theta_{1}, \theta_{2}\right), \partial \Omega\right)>2 \varepsilon$ for all $\left(\theta_{1}, \theta_{2}\right) \in \mathbb{D}_{\Theta}$,

(iii) $\|\varphi\|_{\mathbf{W}^{3, \infty}(\Omega)} \leq C\|\varphi\|_{\mathbf{W}^{3, \infty}\left(S_{s}\right)}$, for some $C>0$.

Proof. Extension results are classical, we can for instance find an extension result for smooth domains in [19, Lemma 12.2]. We can get the present result by multiplying the extension function of [19, Lemma 12.2] by a cut-off function in $\mathcal{D}\left(\Omega_{\varepsilon}\right)$. Note that the existence of $\varepsilon>0$ fulfilling $(i i)$ is a consequence of Assumption $(1.4)$.

Let us denote Id the identity function, we then define the following function

$$
\mathbf{\Phi}\left(\theta_{1}, \theta_{2}, \mathbf{y}\right)=\mathbf{y}+\mathcal{E}\left(\mathbf{X}\left(\theta_{1}, \theta_{2}, .\right)-\mathrm{Id}\right)(\mathbf{y}), \quad \forall\left(\theta_{1}, \theta_{2}\right) \in \mathbb{D}_{\Theta}, \quad \forall \mathbf{y} \in \Omega
$$

We have $\nabla \boldsymbol{\Phi}(0,0, \mathbf{y})=\mathrm{I}$, the identity matrix in $\mathbb{R}^{2 \times 2}$, for every $\mathbf{y} \in \Omega$, hence $\operatorname{det}(\nabla \boldsymbol{\Phi}(0,0, \mathbf{y}))=1$. Then, we can restrict $\mathbb{D}_{\Theta}$ such that for every $\left(\theta_{1}, \theta_{2}\right) \in \mathbb{D}_{\Theta}$, the function $\boldsymbol{\Phi}\left(\theta_{1}, \theta_{2},.\right)$ is a diffeomorphism close to the identity function. We denote $\boldsymbol{\Psi}\left(\theta_{1}, \theta_{2},.\right)$ the inverse diffeomorphism of $\boldsymbol{\Phi}\left(\theta_{1}, \theta_{2},.\right)$

$$
\forall\left(\theta_{1}, \theta_{2}\right) \in \mathbb{D}_{\Theta}, \quad \forall \mathbf{y} \in \Omega, \quad \boldsymbol{\Psi}\left(\theta_{1}, \theta_{2}, \mathbf{\Phi}\left(\theta_{1}, \theta_{2}, \mathbf{y}\right)\right)=\mathbf{y} .
$$

If needed, we can once more reduce $\mathbb{D}_{\Theta}$ to prove that $\boldsymbol{\Phi}$ and $\boldsymbol{\Psi}$ belong to $\mathscr{C}^{\infty}\left(\mathbb{D}_{\Theta}, \mathbf{W}^{3, \infty}(\Omega)\right)$. These diffeomorphisms are represented in Fig. 2.

The properties of $\mathcal{E}$ imply that

$$
\text { for every }\left(\theta_{1}, \theta_{2}\right) \in \mathbb{D}_{\Theta}, \quad \boldsymbol{\Phi}\left(\theta_{1}, \theta_{2}, S_{s}\right)=S\left(\theta_{1}, \theta_{2}\right) \quad \text { and } \quad \forall \mathbf{y} \in \Omega \backslash \Omega_{\varepsilon}, \quad \boldsymbol{\Phi}\left(\theta_{1}, \theta_{2}, \mathbf{y}\right)=\mathbf{y},
$$

where $\Omega_{\varepsilon}$ is defined in Lemma 1.4 .

The stabilization problem. In order to prove a stabilization result on the nonlinear problem, we first study the linearized problem around $\left(\mathbf{w}, p_{\mathbf{w}}, 0,0\right)$ and prove its stabilizability. It requires the technical hypothesis $(\mathcal{H})_{\delta}$ that is presented hereafter.

In the sequel, $\mathbf{v}$ can be thought of as the difference between the state $\mathbf{u}$ and the stationary state $\mathbf{w}$ of the problem (see (3.1) for its precise definition). The linearized term in $\mathbf{v}$ in the fluid equation is the usual Oseen term $(\mathbf{v} \cdot \nabla) \mathbf{w}+(\mathbf{w} \cdot \nabla) \mathbf{v}$. The linearized term in $\left(\theta_{1}, \theta_{2}, \dot{\theta}_{1}, \dot{\theta}_{2}\right)$ in the fluid equation is denoted $\mathbf{L}_{\mathbf{F}}$. In the same way, we denote $\mathbf{L}_{\mathbf{S}}$ the linearized term in $\left(\theta_{1}, \theta_{2}\right)$ in the structure equation. Then we have

$$
\mathbf{L}_{\mathbf{F}}\left(\theta_{1}, \theta_{2}, \dot{\theta}_{1}, \dot{\theta}_{2}, \mathbf{y}\right)=\mathbf{L}_{1}(\mathbf{y}) \theta_{1}+\mathbf{L}_{2}(\mathbf{y}) \theta_{2}+\mathbf{L}_{3}(\mathbf{y}) \dot{\theta}_{1}+\mathbf{L}_{4}(\mathbf{y}) \dot{\theta}_{2}, \quad \forall \mathbf{y} \in \mathscr{F}_{s}
$$

and

$$
\mathbf{L}_{\mathbf{S}}\left(\theta_{1}, \theta_{2}\right)=\mathbf{L}_{5} \theta_{1}+\mathbf{L}_{6} \theta_{2}
$$

where the exact expressions of the coefficients $\mathbf{L}_{1}-\mathbf{L}_{6}$ are given in Appendix A. The coefficients $\mathbf{L}_{1}-\mathbf{L}_{4}$ are functions and $\mathbf{L}_{5}-\mathbf{L}_{6}$ are constant vectors of $\mathbb{R}^{2}$. They all depend on the non-null stationary state (w, $p_{\mathbf{w}}$ ) which is solution of (1.17), on the diffeomorphism $\boldsymbol{\Phi}$ and on its derivatives taken in $\left(\theta_{1}, \theta_{2}\right)=(0,0)$.

Let $\delta>0$ be a prescribed exponential decay rate in time for the difference between the solution and the stationary state. In order to prove the main result of the study, we need the following assumption that depends on $\delta$ and corresponds to a Hautus test. 
Hypothesis $(\mathcal{H})_{\delta}$ (A unique continuation property). Every eigenvector $\left(\mathbf{v}, q, \theta_{1}, \theta_{2}, \omega_{1}, \omega_{2}\right) \in \mathbf{H}^{1}\left(\mathscr{F}_{s}\right) \times$ $\mathrm{L}^{2}\left(\mathscr{F}_{s}\right) \times \mathbb{R}^{4}$ of the adjoint problem associated to the eigenvalue $\bar{\lambda}$ with $\mathcal{R} e(\bar{\lambda}) \geq-\delta$, i.e. every solution of

$$
\begin{cases}\operatorname{div} \sigma_{F}(\mathbf{v}, q)-(\nabla \mathbf{w})^{T} \mathbf{v}+(\mathbf{w} \cdot \nabla) \mathbf{v}=\bar{\lambda} \mathbf{v} & \begin{array}{l}
\text { in } \mathscr{F}_{s}, \\
\operatorname{div} \mathbf{v}=0
\end{array} \\
\mathbf{v}=\omega_{1} \partial_{\theta_{1}} \mathbf{\Phi}(0,0, .)+\omega_{2} \partial_{\theta_{2}} \mathbf{\Phi}(0,0, .) & \begin{array}{l}
\text { on } \partial S_{s}, \\
\text { on } \Gamma_{\mathrm{D}}, \\
\text { on } \Gamma_{\mathrm{N}},
\end{array} \\
\mathbf{v}=0 & \\
\sigma_{F}(\mathbf{v}, q) \mathbf{n}+(\mathbf{w} \cdot \mathbf{n}) \mathbf{v}=0 & \\
\left.\int_{\mathscr{F}_{s}}\left(\begin{array}{c}
\mathbf{L}_{1}(\mathbf{y}) \cdot \mathbf{v}(\mathbf{y}) \\
\mathbf{L}_{2}(\mathbf{y}) \cdot \mathbf{v}(\mathbf{y})
\end{array}\right) \mathrm{d} \mathbf{y}+\left(\begin{array}{l}
\mathbf{L}_{5} \cdot\left(\begin{array}{c}
\omega_{1} \\
\omega_{2}
\end{array}\right) \\
\omega_{1} \\
\omega_{2}
\end{array}\right)\right)=\bar{\lambda}\left(\begin{array}{c}
\theta_{1} \\
\theta_{2}
\end{array}\right), \\
\int_{\mathscr{F}_{s}}\left(\begin{array}{c}
\mathbf{L}_{3}(\mathbf{y}) \cdot \mathbf{v}(\mathbf{y}) \\
\mathbf{L}_{4}(\mathbf{y}) \cdot \mathbf{v}(\mathbf{y})
\end{array}\right) \mathrm{d} \mathbf{y}-\int_{\partial S_{s}}\left(\begin{array}{c}
\sigma_{F}(\mathbf{v}, q) \mathbf{n}_{s}\left(\gamma_{y}\right) \cdot \partial_{\theta_{1}} \mathbf{\Phi}\left(0,0, \gamma_{y}\right) \\
\sigma_{F}(\mathbf{v}, q) \mathbf{n}_{s}\left(\gamma_{y}\right) \cdot \partial_{\theta_{2}} \mathbf{\Phi}\left(0,0, \gamma_{y}\right)
\end{array}\right) \mathrm{d} \gamma_{y}+\left(\begin{array}{c}
\theta_{1} \\
\theta_{2}
\end{array}\right)=\bar{\lambda} \mathcal{M}_{0,0}\left(\begin{array}{c}
\omega_{1} \\
\omega_{2}
\end{array}\right),\end{cases}
$$

that belongs to the kernel of the adjoint of the control operator, i.e. that satisfies

$$
\left\{\begin{array}{l}
\omega_{1}=0 \\
\omega_{2}=0
\end{array}\right.
$$

is necessarily null, i.e. $\left(\mathbf{v}, q, \theta_{1}, \theta_{2}, \omega_{1}, \omega_{2}\right)=(0,0,0,0,0,0)$.

This hypothesis is a unique continuation property for the adjoint system. Such a property is proven for some problems, in particular for the Stokes problem with localized observation [12]. However, in our case of study, the observation is nonlocal, and to our knowledge the corresponding unique continuation property is not available in the literature. In order to lead the study of the stabilization of our problem, we assume this unique continuation property to be valid. Although we do not know how to prove it, we can reasonably think that it is generically valid. Besides, it can be checked numerically on each particular instance.

Remark 1.5. Hypothesis $(\mathcal{H})_{\delta}$ is independent from the choice of the extension operator $\mathcal{E}$ used in Lemma 1.4 to construct the diffeomorphism $\boldsymbol{\Phi}$, see Appendix B.

In the sequel, $\mathcal{J}_{\boldsymbol{\Phi}}\left(\theta_{1}, \theta_{2},.\right)$ denotes the Jacobian matrix of $\boldsymbol{\Phi}\left(\theta_{1}, \theta_{2},.\right)$ and $\operatorname{cof}\left(\mathcal{J}_{\boldsymbol{\Phi}}\left(\theta_{1}, \theta_{2},.\right)\right)$ its cofactor matrix. The goal of the study is to prove the following theorem.

Theorem 1.6 (Main result). Let $\delta>0$ and assume that $(\mathcal{H})_{\delta}$ is fulfilled. Let $\mathbf{f}_{\mathscr{F}} \in \mathbf{W}^{1, \infty}(\Omega), \mathbf{u}^{i} \in \mathbf{U}^{i}$, $\mathbf{f}_{\mathbf{s}} \in \mathbb{R}^{2}$, and $\left(\mathbf{w}, p_{\mathbf{w}}\right) \in \mathbf{H}^{3 / 2}\left(\mathscr{F}_{s}\right) \times \mathrm{H}^{1 / 2}\left(\mathscr{F}_{s}\right)$ fulfil $(1.17)$. Then, there exists $\varepsilon>0$ such that for every $\left(\mathbf{u}_{0}, \theta_{1,0}, \theta_{2,0}, \omega_{1,0}, \omega_{2,0}\right) \in \mathbf{H}^{1}\left(\mathscr{F}\left(\theta_{1,0}, \theta_{2,0}\right)\right) \times \mathbb{D}_{\Theta} \times \mathbb{R}^{2}$ satisfying the compatibility conditions

$$
\begin{cases}\operatorname{div} \mathbf{u}_{0}=0 & \text { in } \mathscr{F}\left(\theta_{1,0}, \theta_{2,0}\right), \\ \mathbf{u}_{0}(.)=\sum_{j=1}^{2} \omega_{j, 0} \partial_{\theta_{j}} \mathbf{X}\left(\theta_{1,0}, \theta_{2,0}, \mathbf{Y}\left(\theta_{1,0}, \theta_{2,0}, .\right)\right) & \text { on } \partial S\left(\theta_{1,0}, \theta_{2,0}\right), \\ \mathbf{u}_{0}=\mathbf{u}^{i} & \text { on } \Gamma_{\mathrm{i}}, \\ \mathbf{u}_{0}=0 & \text { on } \Gamma_{\mathrm{w}},\end{cases}
$$

and

$$
\left\|\mathbf{u}_{0}\left(\boldsymbol{\Phi}\left(\theta_{1,0}, \theta_{2,0}, .\right)\right)-\mathbf{w}(.)\right\|_{\mathbf{H}^{1}\left(\mathscr{F}_{s}\right)}+\left|\theta_{1,0}\right|+\left|\theta_{2,0}\right|+\left|\omega_{1,0}\right|+\left|\omega_{2,0}\right| \leq \varepsilon,
$$

there exists a control $\mathbf{h}$ given under the feedback form

$$
\mathbf{h}(t)=\mathcal{K}_{\delta}\left(\left[\operatorname{cof}\left(\mathcal{J}_{\boldsymbol{\Phi}}\left(\theta_{1}(t), \theta_{2}(t), .\right)\right)^{T} \mathbf{u}\left(t, \boldsymbol{\Phi}\left(\theta_{1}(t), \theta_{2}(t), .\right)\right)-\mathbf{w}\right], \theta_{1}(t), \theta_{2}(t), \dot{\theta}_{1}(t), \dot{\theta}_{2}(t)\right),
$$

for some linear operator $\mathcal{K}_{\delta} \in \mathcal{L}\left(\mathbf{L}^{2}\left(\mathscr{F}_{s}\right) \times \mathbb{R}^{4}, \mathbb{R}^{2}\right)$ such that a solution $\left(\mathbf{u}, p, \theta_{1}, \theta_{2}\right)$ to problem (1.15) fulfils for all $t$ in $(0, \infty)$

$$
\left\|\mathbf{u}\left(t, \boldsymbol{\Phi}\left(\theta_{1}(t), \theta_{2}(t), .\right)\right)-\mathbf{w}(.)\right\|_{\mathbf{H}^{1}\left(\mathscr{F}_{s}\right)}+\left|\theta_{1}(t)\right|+\left|\theta_{2}(t)\right|+\left|\dot{\theta}_{1}(t)\right|+\left|\dot{\theta}_{2}(t)\right| \leq C e^{-\delta t},
$$

for some $C>0$ depending on the geometry, on $\delta$ and on the initial and nonhomogeneous data.

Theorem 1.6 is proven in Sections 2 and 3.

Remark 1.7. The feedback law proposed in (1.25) does not depend linearly on the state $\left(\mathbf{u}, p, \theta_{1}, \theta_{2}\right)$. 


\subsection{The functional framework}

In this section we present the functional setting used in the sequel. We denote by $\mathscr{C}^{0}([0, \infty) ; \mathbb{X})$ the set of functions that are continuous on $[0, \infty)$ and valued in $\mathbb{X}$.

Sobolev spaces. We denote $\mathrm{H}^{r}\left(\mathscr{F}_{s}\right)$ the usual Sobolev space of order $r \geq 0$. We identify $\mathrm{L}^{2}\left(\mathscr{F}_{s}\right)$ with $\mathrm{H}^{0}\left(\mathscr{F}_{s}\right)$. We will denote $\mathbf{L}^{2}\left(\mathscr{F}_{s}\right)=\left(\mathrm{L}^{2}\left(\mathscr{F}_{s}\right)\right)^{2}, \mathbf{H}^{r}\left(\mathscr{F}_{s}\right)=\left(\mathrm{H}^{r}\left(\mathscr{F}_{s}\right)\right)^{2}$ and so on.

Corners issues. The domain considered for the fluid has four corners of angle $\pi / 2$. The ones that are located between Dirichlet and Neumann boundary conditions induce singularities, we denote them $A=(L, 1)$ and $B=(L, 0)$ (see Fig. 1). We also denote $\mathscr{J}_{d, n}=\{A, B\}$ the set of these corners and we define the distance of a point $\mathbf{x}$ from these corners

$$
\text { for } j \in \mathscr{J}_{d, n}, \quad \text { and for } \mathbf{x} \in \Omega, \quad r_{j}(\mathbf{x})=\mathrm{d}(\mathbf{x}, j) \text {. }
$$

Note that corners between two Dirichlet boundary conditions do not induce singularities as soon as suitable compatibility conditions are satisfied. We report to [20, Chapter 9] for more details.

Weighted Sobolev spaces. The solution to the Stokes problem in the domain with corners $A$ and $B$ and with a source term in $\mathbf{L}^{2}\left(\mathscr{F}_{s}\right)$ belongs to a classical Sobolev space of lower order than the one we usually have in smooth domains. In order to get the usual gain of regularity between solutions and source terms, we have to study the solution in adapted Sobolev spaces that are suitably weighted near corners $A$ and $B$. The weighted Sobolev spaces are then defined for $\beta>0$ by

$$
\mathbf{H}_{\beta}^{2}\left(\mathscr{F}_{s}\right)=\left\{\mathbf{u} \text { with }\|\mathbf{u}\|_{\mathbf{H}_{\beta}^{2}\left(\mathscr{F}_{s}\right)}<+\infty\right\}, \quad \mathrm{H}_{\beta}^{1}\left(\mathscr{F}_{s}\right)=\left\{p \text { with }\|p\|_{\mathrm{H}_{\beta}^{1}\left(\mathscr{F}_{s}\right)}<+\infty\right\},
$$

where the norms $\|\cdot\|_{\mathbf{H}_{\beta}^{2}\left(\mathscr{F}_{s}\right)}$ and $\|\cdot\|_{\mathrm{H}_{\beta}^{1}\left(\mathscr{F}_{s}\right)}$ are given by

$$
\|\mathbf{u}\|_{\mathbf{H}_{\beta}^{2}\left(\mathscr{F}_{s}\right)}^{2}=\sum_{|\alpha|=0}^{2} \sum_{i=1}^{2} \int_{\mathscr{F}_{s}}\left(\prod_{j \in \mathscr{J}_{d, n}} r_{j}^{2 \beta}(\mathbf{y})\right)\left|\partial^{\alpha} u_{i}(\mathbf{y})\right|^{2} \mathrm{~d} \mathbf{y},
$$

and

$$
\|p\|_{\mathrm{H}_{\beta}^{1}\left(\mathscr{F}_{s}\right)}^{2}=\sum_{|\alpha|=0}^{1} \int_{\mathscr{F}_{s}}\left(\prod_{j \in \mathscr{J}_{d, n}} r_{j}^{2 \beta}(\mathbf{y})\right)\left|\partial^{\alpha} p(\mathbf{y})\right|^{2} \mathrm{~d} \mathbf{y} .
$$

Here the sum is on every multi-index $\alpha$ of length $|\alpha| \leq 2$ for (1.27), $|\alpha| \leq 1$ for (1.28) and $r_{j}$ is defined in (1.26).

Steady Stokes problem with corners. The following lemma from [23] explains how and why the spaces $\mathbf{H}_{\beta}^{2}\left(\mathscr{F}_{s}\right)$ and $\mathrm{H}_{\beta}^{1}\left(\mathscr{F}_{s}\right)$ appear in the presence of corners. It gives the expected result for the steady Stokes problem in $\mathscr{F}_{s}$ with weigthed Sobolev spaces and the regularity obtained in the classical Sobolev spaces.

Lemma 1.8. [23, Theorem 2.5.] Let us assume that $\mathbf{f}_{\mathscr{F}} \in \mathbf{L}^{2}\left(\mathscr{F}_{s}\right)$. The unique solution $(\mathbf{u}, p)$ to the Stokes problem

$$
\begin{cases}-\operatorname{div} \sigma_{F}(\mathbf{u}, p)=\mathbf{f}_{\mathscr{F}} & \text { in } \mathscr{F}_{s}, \\ \operatorname{div} \mathbf{u}=0 & \text { in } \mathscr{F}_{s}, \\ \mathbf{u}=0 & \text { on } \Gamma_{\mathrm{D}} \cup \partial S_{s}, \\ \sigma_{F}(\mathbf{u}, p) \mathbf{n}=0 & \text { on } \Gamma_{\mathrm{N}},\end{cases}
$$

belongs to $\mathbf{H}_{\beta}^{2}\left(\mathscr{F}_{s}\right) \times \mathrm{H}_{\beta}^{1}\left(\mathscr{F}_{s}\right)$ for some $\beta \in(0,1 / 2)$ and to $\mathbf{H}^{3 / 2+\varepsilon_{0}}\left(\mathscr{F}_{s}\right) \times \mathrm{H}^{1 / 2+\varepsilon_{0}}\left(\mathscr{F}_{s}\right)$ for some $\varepsilon_{0} \in(0,1 / 2)$. Moreover, we have the following estimate

$$
\|\mathbf{u}\|_{\mathbf{H}_{\beta}^{2}\left(\mathscr{F}_{s}\right) \cap \mathbf{H}^{3 / 2+\varepsilon_{0}\left(\mathscr{F}_{s}\right)}}+\|p\|_{\mathrm{H}_{\beta}^{1}\left(\mathscr{F}_{s}\right) \cap \mathrm{H}^{1 / 2+\varepsilon_{0}\left(\mathscr{F}_{s}\right)}} \leq C\left\|\mathbf{f}_{\mathscr{F}}\right\|_{\mathbf{L}^{2}\left(\mathscr{F}_{s}\right)} .
$$

Remark 1.9. A consequence of Lemma 1.8 is that the solution $\left(\mathbf{w}, p_{\mathbf{w}}\right)$ of $(1.17)-(1.18)$ belongs to $\mathbf{H}_{\beta}^{2}\left(\mathscr{F}_{s}\right) \times$ $\mathrm{H}_{\beta}^{1}\left(\mathscr{F}_{s}\right)$. This is the regularity that we will use for $\left(\mathbf{w}, p_{\mathbf{w}}\right)$ in the sequel. A proof of this statement can be achieved by lifting the datum $\mathbf{u}^{i}$, which is done in [11, Lemma 2.10].

Keep in mind that $\mathbf{n}_{s}$ is the outward unit normal to $\mathscr{F}_{s}$. Note that, according to the regularity proven in Lemma 1.8, the traces $p_{\mid \partial \mathscr{F}_{s}}$ and $\partial_{\mathbf{n}_{s}} \mathbf{u}_{\mid \partial \mathscr{F}_{s}}$ are well defined, which gives a meaning to all integrations by parts.

Also note that according to [17, Theorem 1.4.3.1], there exists a continuous extension operator from $\mathbf{H}^{s}\left(\mathscr{F}_{s}\right)$ to $\mathbf{H}^{s}\left(\mathbb{R}^{2}\right)$ for every $s>0$. This implies that all the classical Sobolev embeddings and interpolations are valid despite the presence of corners. 


\subsection{Scientific context}

There are several works providing stabilization results in the context of the Navier-Stokes equations. For instance, the stabilization of a viscous fluid is treated for the wake of a cylinder in [14, 15, 16, 23] and for a cavity in [21]. A first strategy used in [15, 16, 21] reposes on the Proper Orthogonal Decomposition (POD) approach. Another approach consists in constructing a feedback operator by means of a Riccati equation making the closed-loop system stable [23, 25, 26]. If needed, it is possible to use dynamical controllers to meet compatibility conditions between the fluid initial datum and the initial control value, the control is then computed as the solution of an ODE [1, 2].

When we consider fluid-structure interaction problems the same strategies can be used. The reader can refer for instance to [28] for the stabilization by a POD approach of a fluid around an airfoil. It is also possible to build a stabilizing feedback control that uses only the state of the structure, see [7] for a 1D and [29] for a 2D fluid-solid interaction problems.

In the present study, we use a stabilizing control that is given under a feedback form and uses the state of the fluid and the structure. The feedback operator is computed via the solution of a finite dimensional Riccati equation. This is helpful when treating numerical simulations which are not the point of the current paper and are a work currently in progress. The same strategy has already been used to prove stabilization of strong solutions, which is what we aim for, and more recently stabilization of weak solutions to a fluid-beam interaction problem [5]. In the literature, the feedback control can be a Dirichlet datum imposed to the fluid on some part of the boundary $[3,22]$, it can be a change in the shape of the structure $[8,9]$ or a force acting on the structure $[22,27]$.

Although the control that we use in the current study acts on the structure, the study [3] is the closest one from what we want to prove. It treats the stabilization of a fluid-rigid body system by a feedback control law acting on the boundary of the fluid domain. In the current study, we follow its framework and account for the deformability of the structure and the control acting on the acceleration of the structure. Additional difficulties are induced by the corners on $\partial \Omega$, more information about them can be found in $[20,23]$.

\subsection{Outline of the paper}

In Section 2, we prove the existence of a feedback control law that stabilizes the solution of the linearized system in the fixed domain $\mathscr{F}_{s}$ around the stationary state $\left(\mathbf{w}, p_{\mathbf{w}}, 0,0\right)$. The proof relies on the analysis of the properties of the linearized system. In Section 3, we extend successively the previous result to the full nonlinear system in the fixed domain and in the moving domain. The former is proven via a fixed point argument and the latter uses a change of variables. The linearized terms are summed up in Appendix A. An idea of the proof of Remark 1.5 is given in Appendix B. The proof of the technical estimates of the nonlinear terms can be found in Appendix C.

Acknowledgement: The author wants to thank Mehdi Badra for fruitful discussions.

\section{Stabilization of the linearized problem}

In the whole Section 2 we consider stationary nonhomogeneous terms $\left(\mathbf{f}_{\mathscr{F}}, \mathbf{u}^{i}, \mathbf{f}_{\mathbf{s}}\right) \in \mathbf{W}^{1, \infty}(\Omega) \times \mathbf{U}^{i} \times \mathbb{R}^{2}$ and a stationary state $\left(\mathbf{w}, p_{\mathbf{w}}\right) \in \mathbf{H}_{\beta}^{2}\left(\mathscr{F}_{s}\right) \times \mathrm{H}_{\beta}^{1}\left(\mathscr{F}_{s}\right)$ that fulfil (1.17). Our goal is to find a control law $\mathbf{h}$ under a feedback form that stabilizes the linearized problem with a given exponential decay $\delta$ in time.

\subsection{The linearized problem}

We study the linearized system associated to (1.15). The variables $(\mathbf{v}, q)$ correspond roughly to the difference between $(\mathbf{u}, p)$ written in the fixed domain $\mathscr{F}_{s}$ and $\left(\mathbf{w}, p_{\mathbf{w}}\right)$. A proper definition of these variables can be found in (3.1). Here is the linearized system around $\left(\mathbf{w}, p_{\mathbf{w}}, 0,0\right)$ 


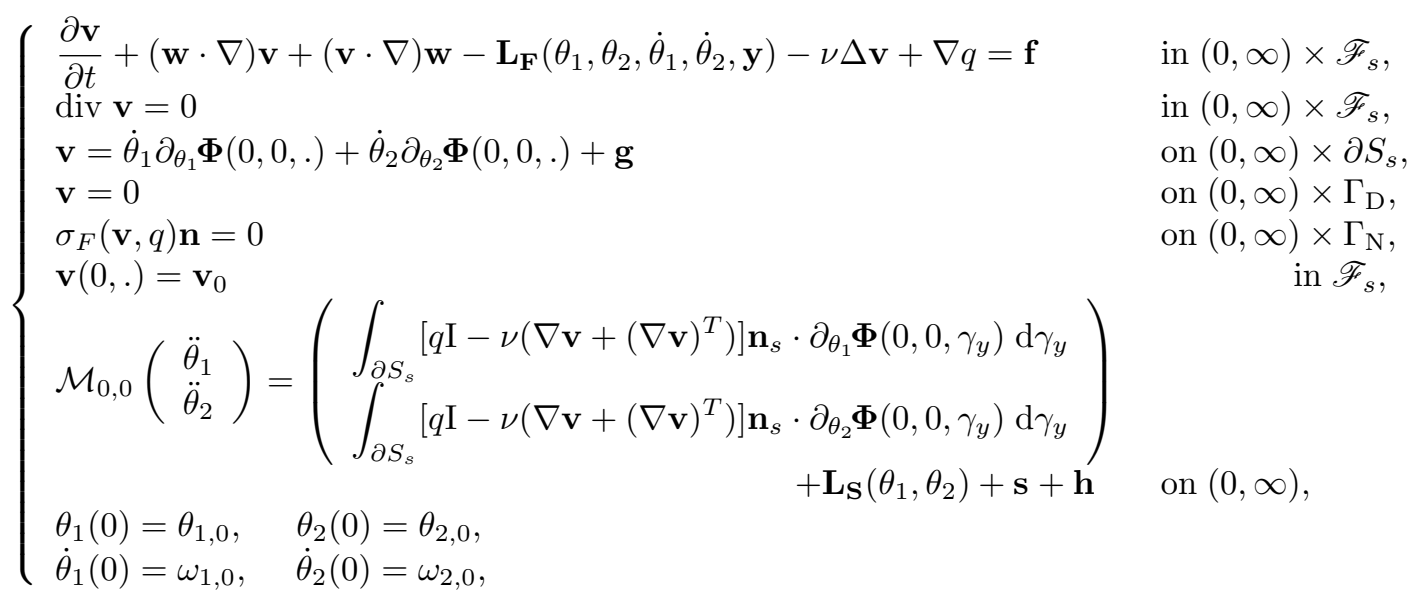

where $(\mathbf{f}, \mathbf{g}, \mathbf{s})$ are nonhomogeneous terms and $\mathbf{v}_{0}$ an initial datum for $\mathbf{v}$. Here, $\mathbf{L}_{\mathbf{F}} \in \mathbf{L}^{2}\left(\mathscr{F}_{s}, \mathcal{L}\left(\mathbb{R}^{4}, \mathbb{R}^{2}\right)\right)$ and $\mathbf{L}_{\mathbf{S}} \in \mathcal{L}\left(\mathbb{R}^{2}, \mathbb{R}^{2}\right)$ depend on the stationary state $\left(\mathbf{w}, p_{\mathbf{w}}\right)$ and on the diffeomorphism $\boldsymbol{\Phi}$, they are given by

$$
\begin{aligned}
\mathbf{L}_{\mathbf{F}}\left(\theta_{1}, \theta_{2}, \dot{\theta}_{1}, \dot{\theta}_{2}, \mathbf{y}\right) & =\mathbf{L}_{1}(\mathbf{y}) \theta_{1}+\mathbf{L}_{2}(\mathbf{y}) \theta_{2}+\mathbf{L}_{3}(\mathbf{y}) \dot{\theta}_{1}+\mathbf{L}_{4}(\mathbf{y}) \dot{\theta}_{2}, \quad \forall \mathbf{y} \in \mathscr{F}_{s}, \\
\mathbf{L}_{\mathbf{S}}\left(\theta_{1}, \theta_{2}\right) & =\mathbf{L}_{5} \theta_{1}+\mathbf{L}_{6} \theta_{2},
\end{aligned}
$$

where the coefficients $\mathbf{L}_{1}-\mathbf{L}_{6}$ are properly defined in Appendix A ((A.1)-(A.6)).

For any fixed $\delta>0$ such that $(\mathcal{H})_{\delta}$ holds, we use the following spaces

$$
\begin{aligned}
\mathbb{U}_{\delta}^{\infty} & =\left\{\mathbf{v} \text { with } e^{\delta t} \mathbf{v} \in \mathrm{L}^{2}\left(0, \infty ; \mathbf{H}_{\beta}^{2}\left(\mathscr{F}_{s}\right)\right) \cap \mathscr{C}^{0}\left([0, \infty) ; \mathbf{H}^{1}\left(\mathscr{F}_{s}\right)\right) \cap \mathrm{H}^{1}\left(0, \infty ; \mathbf{L}^{2}\left(\mathscr{F}_{s}\right)\right)\right\}, \\
\mathbb{P}_{\delta}^{\infty} & =\left\{q \text { with } e^{\delta t} q \in \mathrm{L}^{2}\left(0, \infty ; \mathrm{H}_{\beta}^{1}\left(\mathscr{F}_{s}\right)\right)\right\}, \\
\Theta_{\delta}^{\infty} & =\left\{\left(\theta_{1}, \theta_{2}\right) \text { with } e^{\delta t}\left(\theta_{1}, \theta_{2}\right) \in \mathrm{H}^{2}\left(0, \infty ; \mathbb{R}^{2}\right)\right\}, \\
\mathbb{F}_{\delta}^{\infty} & =\left\{\mathbf{f} \text { with } e^{\delta t} \mathbf{f} \in \mathrm{L}^{2}\left(0, \infty ; \mathbf{L}^{2}\left(\mathscr{F}_{s}\right)\right)\right\}, \\
\mathbb{G}_{\delta}^{\infty} & =\left\{\mathbf{g} \text { with } e^{\delta t} \mathbf{g} \in \mathrm{H}^{1}\left(0, \infty ; \mathbf{H}^{3 / 2}\left(\partial S_{s}\right)\right)\right\}, \\
\mathbb{S}_{\delta}^{\infty} & =\left\{\mathbf{s} \text { with } e^{\delta t} \mathbf{s} \in \mathrm{L}^{2}\left(0, \infty ; \mathbb{R}^{2}\right)\right\} .
\end{aligned}
$$

All these spaces are equipped with their natural norms, e.g. for $\Theta_{\delta}^{\infty}$,

$$
\left\|\left(\theta_{1}, \theta_{2}\right)\right\|_{\Theta_{\delta}^{\infty}}=\left\|\left(\theta_{1}, \theta_{2}\right) e^{\delta t}\right\|_{\mathrm{H}^{2}\left(0, \infty ; \mathbb{R}^{2}\right)} .
$$

The goal of Section 2 is to prove the following result.

Proposition 2.1. Let $\delta>0$ and assume that $(\mathcal{H})_{\delta}$ is fulfilled. There exists a feedback operator $\mathcal{K}_{\delta} \in \mathcal{L}\left(\mathbf{L}^{2}\left(\mathscr{F}_{s}\right) \times\right.$ $\left.\mathbb{R}^{4}, \mathbb{R}^{2}\right)$, such that for every $\left(\mathbf{v}_{0}, \theta_{1,0}, \theta_{2,0}, \omega_{1,0}, \omega_{2,0}\right) \in \mathbf{H}^{1}\left(\mathscr{F}_{s}\right) \times \mathbb{R}^{4}, \mathbf{f} \in \mathbb{F}_{\delta}^{\infty}, \mathbf{g} \in \mathbb{G}_{\delta}^{\infty}$ and $\mathbf{s} \in \mathbb{S}_{\delta}^{\infty}$ fulfilling the compatibility conditions

$$
\begin{cases}\operatorname{div} \mathbf{v}_{0}=0 & \text { in } \mathscr{F}_{s} \\ \mathbf{v}_{0}=\sum_{j=1}^{2} \omega_{j, 0} \partial_{\theta_{j}} \mathbf{\Phi}(0,0, .)+\mathbf{g}(0) & \text { on } \partial S_{s} \\ \mathbf{v}_{0}=0 & \text { on } \Gamma_{\mathrm{D}}\end{cases}
$$

problem (2.1) with the control taken as $\mathbf{h}=\mathcal{K}_{\delta}\left(\mathbf{v}, \theta_{1}, \theta_{2}, \dot{\theta}_{1}, \dot{\theta}_{2}\right)$ admits a unique solution $\left(\mathbf{v}, q, \theta_{1}, \theta_{2}\right) \in \mathbb{U}_{\delta}^{\infty} \times$ $\mathbb{P}_{\delta}^{\infty} \times \Theta_{\delta}^{\infty}$ with the following estimate,

$$
\|\mathbf{v}\|_{\mathrm{U}_{\delta}^{\infty}}+\|q\|_{\mathbb{P}_{\delta}^{\infty}}+\left\|\left(\theta_{1}, \theta_{2}\right)\right\|_{\Theta_{\delta}^{\infty}} \leq C\left(\left\|\mathbf{v}_{0}\right\|_{\mathbf{H}^{1}\left(\mathscr{F}_{s}\right)}+\left|\theta_{1,0}\right|+\left|\theta_{2,0}\right|+\left|\omega_{1,0}\right|+\left|\omega_{2,0}\right|+\|\mathbf{f}\|_{\mathbb{F}_{\delta}^{\infty}}+\|\mathbf{g}\|_{\mathbb{G}_{\delta}^{\infty}}+\|\mathbf{s}\|_{\mathcal{S}_{\delta}^{\infty}}\right),
$$

where $C$ does not depend on the initial conditions and on the source terms.

$A$ consequence is that for every $t \in(0, \infty)$,

$$
\begin{aligned}
\|\mathbf{v}(t)\|_{\mathbf{H}^{1}\left(\mathscr{F}_{s}\right)}+\left|\theta_{1}(t)\right|+\left|\theta_{2}(t)\right|+\left|\dot{\theta}_{1}(t)\right|+\left|\dot{\theta}_{2}(t)\right| \leq C\left(\left\|\mathbf{v}_{0}\right\|_{\mathbf{H}^{1}\left(\mathscr{F}_{s}\right)}\right. & +\left|\theta_{1,0}\right|+\left|\theta_{2,0}\right|+\left|\omega_{1,0}\right|+\left|\omega_{2,0}\right| \\
& \left.+\|\mathbf{f}\|_{\mathbf{F}_{\delta}^{\infty}}+\|\mathbf{g}\|_{\mathbb{G}_{\delta}^{\infty}}+\|\mathbf{s}\|_{\mathbb{S}_{\delta}^{\infty}}\right) e^{-\delta t} .
\end{aligned}
$$

We first work on the homogeneous system associated to (2.1). In Section 2.2 we develop the functional framework used to write the homogeneous system under a semigroup formulation. In Section 2.3 we study the adjoint operator. In Section 2.4 we exhibit a feedback operator $\mathcal{K}_{\delta}$ that stabilizes the homogeneous problem. We then prove Proposition 2.1 in Section 2.5. 


\subsection{Functional framework for the semigroup formulation}

In this section, the linear problem considered in Section 2.1 is rewritten under a semigroup formulation. This enables us to use the classical strategy to derive a feedback operator $\mathcal{K}_{\delta}$ stabilizing the system (2.1) (see [6, Part V] and [10, Section 5.2] for a full presentation of this method). In a similar way to [11], we use the spaces

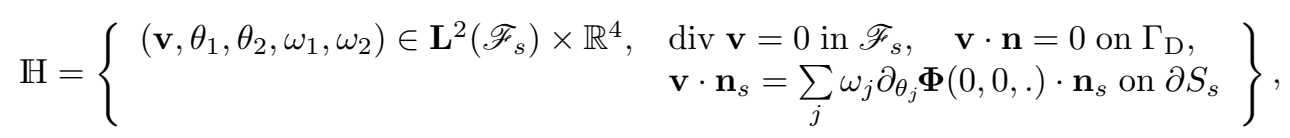

and

$$
\mathbb{V}=\left\{\begin{array}{ll}
\left(\mathbf{v}, \theta_{1}, \theta_{2}, \omega_{1}, \omega_{2}\right) \in \mathbf{H}^{1}\left(\mathscr{F}_{s}\right) \times \mathbb{R}^{4}, & \operatorname{div} \mathbf{v}=0 \text { in } \mathscr{F}_{s}, \quad \mathbf{v}=0 \text { on } \Gamma_{\mathrm{D}} \\
& \mathbf{v}=\sum_{j} \omega_{j} \partial_{\theta_{j}} \mathbf{\Phi}(0,0, .) \text { on } \partial S_{s}
\end{array}\right\}
$$

The spaces $\mathbb{H}$ and $\mathbb{V}$ are respectively endowed with the scalar products $(., .)_{0}$ of $\mathbf{L}^{2}\left(\mathscr{F}_{s}\right) \times \mathbb{R}^{4}$ and $(., .)_{1}$ of $\mathbf{H}^{1}\left(\mathscr{F}_{s}\right) \times \mathbb{R}^{4}$ defined by

$$
\begin{aligned}
& \forall\left(\mathbf{v}^{j}, \theta_{1}^{j}, \theta_{2}^{j}, \omega_{1}^{j}, \omega_{2}^{j}\right) \in \mathbf{L}^{2}\left(\mathscr{F}_{s}\right) \times \mathbb{R}^{4}, \\
& \left(\left(\mathbf{v}^{a}, \theta_{1}^{a}, \theta_{2}^{a}, \omega_{1}^{a}, \omega_{2}^{a}\right),\left(\mathbf{v}^{b}, \theta_{1}^{b}, \theta_{2}^{b}, \omega_{1}^{b}, \omega_{2}^{b}\right)\right)_{0}=\int_{\mathscr{F}_{s}} \mathbf{v}^{a} \cdot \mathbf{v}^{b} \mathrm{~d} \mathbf{y}+\sum_{j} \theta_{j}^{a} \theta_{j}^{b}+\left(\omega_{1}^{a} \omega_{2}^{a}\right) \mathcal{M}_{0,0}\left(\begin{array}{c}
\omega_{1}^{b} \\
\omega_{2}^{b}
\end{array}\right),
\end{aligned}
$$

and

$$
\begin{aligned}
& \forall\left(\mathbf{v}^{j}, \theta_{1}^{j}, \theta_{2}^{j}, \omega_{1}^{j}, \omega_{2}^{j}\right) \in \mathbf{H}^{1}\left(\mathscr{F}_{s}\right) \times \mathbb{R}^{4}, \\
& \left(\left(\mathbf{v}^{a}, \theta_{1}^{a}, \theta_{2}^{a}, \omega_{1}^{a}, \omega_{2}^{a}\right),\left(\mathbf{v}^{b}, \theta_{1}^{b}, \theta_{2}^{b}, \omega_{1}^{b}, \omega_{2}^{b}\right)\right)_{1}=\int_{\mathscr{F}_{s}}\left(\mathbf{v}^{a} \cdot \mathbf{v}^{b}+\nabla \mathbf{v}^{a}: \nabla \mathbf{v}^{b}\right) \mathrm{d} \mathbf{y}+\sum_{j} \theta_{j}^{a} \theta_{j}^{b}+\left(\omega_{1}^{a} \omega_{2}^{a}\right) \mathcal{M}_{0,0}\left(\begin{array}{c}
\omega_{1}^{b} \\
\omega_{2}^{b}
\end{array}\right),
\end{aligned}
$$

where $\mathcal{M}_{0,0}$ is defined in (1.11) for $\theta_{1}=\theta_{2}=0$.

In the sequel, for a better readability, we use the notation

$$
\left(f_{j}\right)_{j=1,2}=\left(\begin{array}{c}
f_{1} \\
f_{2}
\end{array}\right) .
$$

Lemma 2.2. The orthogonal space to $\mathbb{H}$ with respect to the scalar product $(., .)_{0}$ is

$$
(\mathbb{H})^{\perp}=\left\{\left(\nabla p, 0,0,-\mathcal{M}_{0,0}^{-1}\left(\int_{\partial S_{s}} p \mathbf{n}_{s} \cdot \partial_{\theta_{j}} \mathbf{\Phi}\left(0,0, \gamma_{y}\right) \mathrm{d} \gamma_{y}\right)_{j=1,2}\right) \text { with } p \in \mathrm{H}^{1}\left(\mathscr{F}_{s}\right), p=0 \text { on } \Gamma_{\mathrm{N}}\right\} .
$$

Proof. See [11, Lemma 2.3].

We adapt Rellich's compact embedding Theorem to our functional framework.

Lemma 2.3. The embedding from $\mathbb{V}$ into $\mathbb{H}$ is compact.

Proof. The proof is an easy consequence of Rellich's compact embedding Theorem [17, Theorem 1.4.3.2.].

We define the operator $(A, D(A))$ on $\mathbb{H}$ by

$$
\begin{gathered}
D(A)=\left\{\begin{array}{r}
\left(\mathbf{v}, \theta_{1}, \theta_{2}, \omega_{1}, \omega_{2}\right) \in \mathbb{V}, \mathbf{v} \in \mathbf{H}^{3 / 2+\varepsilon_{0}}\left(\mathscr{F}_{s}\right), \exists q \in \mathrm{H}^{1 / 2+\varepsilon_{0}}\left(\mathscr{F}_{s}\right) \text { such that } \\
\operatorname{div} \sigma_{F}(\mathbf{v}, q) \in \mathbf{L}^{2}\left(\mathscr{F}_{s}\right) \text { and } \sigma_{F}(\mathbf{v}, q) \mathbf{n}=0 \text { on } \Gamma_{\mathrm{N}}
\end{array}\right\}, \\
A\left(\begin{array}{c}
\mathbf{v} \\
\theta_{1} \\
\theta_{2} \\
\omega_{1} \\
\omega_{2}
\end{array}\right)=\Pi_{\mathbb{H}}\left(\begin{array}{l}
\operatorname{div} \sigma_{F}(\mathbf{v}, q)+\mathbf{L}_{\mathbf{F}}\left(\theta_{1}, \theta_{2}, \omega_{1}, \omega_{2}, \mathbf{y}\right)-(\mathbf{v} \cdot \nabla) \mathbf{w}-(\mathbf{w} \cdot \nabla) \mathbf{v} \\
\omega_{1} \\
\omega_{2} \\
\mathcal{M}_{0,0}^{-1}\left(\left(\int_{\partial S_{s}}-\sigma_{F}(\mathbf{v}, q) \mathbf{n}_{s} \cdot \partial_{\theta_{j}} \mathbf{\Phi}\left(0,0, \gamma_{y}\right) \mathrm{d} \gamma_{y}\right)_{j=1,2}^{+}+\mathbf{L}_{\mathbf{S}}\left(\theta_{1}, \theta_{2}\right)\right)
\end{array}\right),
\end{gathered}
$$

where $\varepsilon_{0}$ is introduced in Lemma 1.8 and $\Pi_{\mathbb{H}}$ denotes the orthogonal projection of $\mathbf{L}^{2}\left(\mathscr{F}_{s}\right) \times \mathbb{R}^{4}$ onto $\mathrm{H}$. The next lemmas state some properties of $(A, D(A))$.

Remark 2.4. The use of $q$ in the definition of $(A, D(A))$ is useful to guarantee that $\operatorname{div} \sigma_{F}(\mathbf{v}, q)$ belongs to $\mathbf{L}^{2}\left(\mathscr{F}_{s}\right)$ and then that the application of $\Pi_{\mathbb{H}}$ in the right hand-side of $(2.16)$ makes sense.

Lemma 2.5. The operator $A$ is uniquely defined. 
Proof. A similar proof is presented in [11, Lemma 2.5], we need to slightly adapt it in order to take into account the terms coming from the linearization around a stationary solution.

Let $\left(\mathbf{v}, \theta_{1}, \theta_{2}, \omega_{1}, \omega_{2}\right) \in D(A)$ and consider two functions $p, q \in \mathrm{H}^{1 / 2+\varepsilon_{0}}\left(\mathscr{F}_{s}\right)$ satisfying the conditions appearing into the definition of $D(A)$. Then, $\operatorname{div} \sigma_{F}(0, p-q)=-\nabla(p-q) \in \mathbf{L}^{2}\left(\mathscr{F}_{s}\right)$ implies $p-q \in \mathrm{H}^{1}\left(\mathscr{F}_{s}\right)$, and $\sigma_{F}(0, p-q) \mathbf{n}=0$ on $\Gamma_{\mathrm{N}}$ implies $p-q=0$ on $\Gamma_{\mathrm{N}}$.

Now,

$$
\begin{aligned}
\left.\left(\begin{array}{l}
\operatorname{div} \sigma_{F}(\mathbf{v}, p)+\mathbf{L}_{\mathbf{F}}\left(\theta_{1}, \theta_{2}, \omega_{1}, \omega_{2}, \mathbf{y}\right)-(\mathbf{v} \cdot \nabla) \mathbf{w}-(\mathbf{w} \cdot \nabla) \mathbf{v} \\
\omega_{1} \\
\omega_{2} \\
\mathcal{M}_{0,0}^{-1}\left(\left(\int_{\partial S_{s}}-\sigma_{F}(\mathbf{v}, p) \mathbf{n}_{s} \cdot \partial_{\theta_{j}} \mathbf{\Phi}\left(0,0, \gamma_{y}\right) \mathrm{d} \gamma_{y}\right)_{j=1,2} \mathbf{L}_{\mathbf{S}}\left(\theta_{1}, \theta_{2}\right)\right)
\end{array}\right)\right)\left(\begin{array}{l}
\operatorname{div} \sigma_{F}(\mathbf{v}, q)+\mathbf{L}_{\mathbf{F}}\left(\theta_{1}, \theta_{2}, \omega_{1}, \omega_{2}, \mathbf{y}\right)-(\mathbf{v} \cdot \nabla) \mathbf{w}-(\mathbf{w} \cdot \nabla) \mathbf{v} \\
\omega_{1} \\
\omega_{2} \\
\mathcal{M}_{0,0}^{-1}\left(\left(\int_{\partial S_{s}}-\sigma_{F}(\mathbf{v}, q) \mathbf{n}_{s} \cdot \partial_{\theta_{j}} \mathbf{\Phi}\left(0,0, \gamma_{y}\right) \mathrm{d} \gamma_{y}\right)+\mathbf{L}_{\mathbf{S}}\left(\theta_{1}, \theta_{2}\right)\right)
\end{array}\right) \\
=\left(\begin{array}{l}
\nabla(p-q) \\
0 \\
0 \\
-\mathcal{M}_{0,0}^{-1}\left(\int_{\partial S_{s}}(p-q) \mathbf{n}_{s} \cdot \partial_{\theta_{j}} \boldsymbol{\Phi}\left(0,0, \gamma_{y}\right) \mathrm{d} \gamma_{y}\right)_{j=1,2}
\end{array}\right),
\end{aligned}
$$

which belongs to $\mathbb{H}^{\perp}$ according to Lemma 2.2. Therefore $A$ is uniquely defined.

Before going further, let us point out that $D(A)$ can be characterized as follows.

Lemma 2.6. We have

$$
D(A)=\left\{\begin{array}{r}
\left(\mathbf{v}, \theta_{1}, \theta_{2}, \omega_{1}, \omega_{2}\right) \in \mathbb{V}, \mathbf{v} \in \mathbf{H}_{\beta}^{2}\left(\mathscr{F}_{s}\right), \exists q \in \mathrm{H}_{\beta}^{1}\left(\mathscr{F}_{s}\right) \text { such that } \\
\operatorname{div} \sigma_{F}(\mathbf{v}, q) \in \mathbf{L}^{2}\left(\mathscr{F}_{s}\right) \text { and } \sigma_{F}(\mathbf{v}, q) \mathbf{n}=0 \text { on } \Gamma_{\mathrm{N}}
\end{array}\right\} .
$$

Proof. See [11, Lemma 2.6].

We define the bilinear form $\mathrm{a}_{1}$ on $\mathbb{V} \times \mathbb{V}$ for every $\left(\mathbf{v}, \theta_{1}, \theta_{2}, \omega_{1}, \omega_{2}\right)$ and $\left(\mathbf{v}^{b}, \theta_{1}^{b}, \theta_{2}^{b}, \omega_{1}^{b}, \omega_{2}^{b}\right)$ in $\mathbb{V}$ by

$$
\begin{aligned}
\mathrm{a}_{1}\left(\left(\mathbf{v}, \theta_{1}, \theta_{2}, \omega_{1}, \omega_{2}\right),\left(\mathbf{v}^{b}, \theta_{1}^{b}, \theta_{2}^{b}, \omega_{1}^{b}, \omega_{2}^{b}\right)\right)=\frac{\nu}{2} \int_{\mathscr{F}_{s}}\left(\nabla \mathbf{v}+\nabla \mathbf{v}^{T}\right):\left(\nabla \mathbf{v}^{b}+\left(\nabla \mathbf{v}^{b}\right)^{T}\right) \mathrm{d} \mathbf{y} \\
+\int_{\mathscr{F}_{s}}((\mathbf{v} \cdot \nabla) \mathbf{w}+(\mathbf{w} \cdot \nabla) \mathbf{v}) \cdot \mathbf{v}^{b} \mathrm{~d} \mathbf{y} .
\end{aligned}
$$

We define the operator $\left(A_{1}, D\left(A_{1}\right)\right)$ on $\mathbb{H}$ by

$$
D\left(A_{1}\right)=\left\{\mathbf{z} \in \mathbb{V} \text { with } \widetilde{\mathbf{z}} \mapsto \mathrm{a}_{1}(\mathbf{z}, \widetilde{\mathbf{z}}) \text { is } \mathbb{H} \text {-continuous }\right\},
$$

and

$$
\forall \mathbf{z} \in D\left(A_{1}\right), \forall \tilde{\mathbf{z}} \in \mathbb{V},\left(A_{1} \mathbf{z}, \tilde{\mathbf{z}}\right)_{0}=-\mathrm{a}_{1}(\mathbf{z}, \tilde{\mathbf{z}}) .
$$

Lemma 2.7. We have $D\left(A_{1}\right)=D(A)$ and

$$
A_{1}\left(\begin{array}{l}
\mathbf{v} \\
\theta_{1} \\
\theta_{2} \\
\omega_{1} \\
\omega_{2}
\end{array}\right)=\Pi_{\mathbb{H}}\left(\begin{array}{l}
\operatorname{div} \sigma_{F}(\mathbf{v}, q)-(\mathbf{v} \cdot \nabla) \mathbf{w}-(\mathbf{w} \cdot \nabla) \mathbf{v} \\
0 \\
0 \\
\mathcal{M}_{0,0}^{-1}\left(\int_{\partial S_{s}}-\sigma_{F}(\mathbf{v}, q) \mathbf{n}_{s} \cdot \partial_{\theta_{j}} \mathbf{\Phi}\left(0,0, \gamma_{y}\right) \mathrm{d} \gamma_{y}\right)_{j=1,2}
\end{array}\right) .
$$

Proof. This is a straightforward adaptation of [11, Lemma 2.7].

Lemma 2.8. The operator A generates an analytic semigroup on $\mathbb{H}$ and has compact resolvent.

Proof. According to [23, p. 3015], there exists a constant $c>0$ such that for $\lambda>0$ large enough, we have

$$
\forall \mathbf{z} \in \mathbb{V}, \quad \mathrm{a}_{1}(\mathbf{z}, \mathbf{z})+\lambda\|\mathbf{z}\|_{\mathbb{H}}^{2} \geq c\|\mathbf{z}\|_{\mathbb{V}}^{2} .
$$

Moreover, according to [6, Theorem 2.12, p. 115], the estimate (2.18) implies that the operator $A_{1}$ generates an analytic semigroup on $\mathrm{H}$.

Now, as $A-A_{1} \in \mathcal{L}(\mathbb{H})$, according to [24, Corollary 2.2], $A$ generates an analytic semigroup on $\mathbb{H}$.

We have $D(A) \subset \mathbb{V}$ and, according to Lemma 2.3, the embedding from $\mathbb{V}$ into $\mathbb{H}$ is compact. The operator $A$ then has compact resolvent. This ends this proof. 


\subsection{Study of the adjoint operator}

In order to simplify the notations, in the sequel we do not write $\mathrm{d} \mathbf{y}$ or $\mathrm{d} \gamma_{y}$ anymore in the integrals.

Lemma 2.9. The adjoint operator $A_{1}^{*}$ of $A_{1}$ with respect to the scalar product $(., .)_{0}$ is given by

$$
D\left(A_{1}^{*}\right)=\left\{\begin{array}{ll}
\left(\mathbf{v}, \theta_{1}, \theta_{2}, \omega_{1}, \omega_{2}\right) \in \mathbb{V}, & \mathbf{v} \in \mathbf{H}^{3 / 2+\varepsilon_{0}}\left(\mathscr{F}_{s}\right), \quad \exists q \in \mathrm{H}^{1 / 2+\varepsilon_{0}}\left(\mathscr{F}_{s}\right) \text { such that } \\
& \operatorname{div} \sigma_{F}(\mathbf{v}, q) \in \mathbf{L}^{2}\left(\mathscr{F}_{s}\right) \text { and } \sigma_{F}(\mathbf{v}, q) \mathbf{n}+(\mathbf{w} \cdot \mathbf{n}) \mathbf{v}=0 \text { on } \Gamma_{\mathrm{N}}
\end{array}\right\},
$$

and

$$
A_{1}^{*}\left(\begin{array}{c}
\mathbf{v} \\
\theta_{1} \\
\theta_{2} \\
\omega_{1} \\
\omega_{2}
\end{array}\right)=\Pi_{\mathbb{H}}\left(\begin{array}{l}
\operatorname{div} \sigma_{F}(\mathbf{v}, q)-(\nabla \mathbf{w})^{T} \mathbf{v}+(\mathbf{w} \cdot \nabla) \mathbf{v} \\
0 \\
0 \\
\mathcal{M}_{0,0}^{-1}\left(\int_{\partial S_{s}}-\sigma_{F}(\mathbf{v}, q) \mathbf{n}_{s} \cdot \partial_{\theta_{j}} \mathbf{\Phi}\left(0,0, \gamma_{y}\right)\right)_{j=1,2}
\end{array}\right) .
$$

Proof. The adjoint $A_{1}^{*}$ of $A_{1}$ is defined by

$$
D\left(A_{1}^{*}\right)=\left\{\tilde{\mathbf{z}} \in \mathbb{V} \text { with } \mathbf{z} \mapsto \mathrm{a}_{1}(\mathbf{z}, \tilde{\mathbf{z}}) \text { is } \mathbb{H} \text {-continuous }\right\},
$$

and

$$
\forall \mathbf{z} \in \mathbb{V}, \quad \forall \tilde{\mathbf{z}} \in D\left(A_{1}^{*}\right), \quad\left(\mathbf{z}, A_{1}^{*} \tilde{\mathbf{z}}\right)_{0}=-\mathrm{a}_{1}(\mathbf{z}, \tilde{\mathbf{z}}) .
$$

Let us denote

$$
E=\left\{\begin{array}{ll}
\left(\mathbf{v}, \theta_{1}, \theta_{2}, \omega_{1}, \omega_{2}\right) \in \mathbb{V}, & \mathbf{v} \in \mathbf{H}^{3 / 2+\varepsilon_{0}}\left(\mathscr{F}_{s}\right), \quad \exists q \in \mathrm{H}^{1 / 2+\varepsilon_{0}}\left(\mathscr{F}_{s}\right) \text { such that } \\
& \operatorname{div} \sigma_{F}(\mathbf{v}, q) \in \mathbf{L}^{2}\left(\mathscr{F}_{s}\right) \text { and } \sigma_{F}(\mathbf{v}, q) \mathbf{n}+(\mathbf{w} \cdot \mathbf{n}) \mathbf{v}=0 \text { on } \Gamma_{\mathrm{N}}
\end{array}\right\} .
$$

For every $\tilde{\mathbf{z}}=\left(\widetilde{\mathbf{v}}, \widetilde{\theta}_{1}, \widetilde{\theta}_{2}, \widetilde{\omega}_{1}, \widetilde{\omega}_{2}\right) \in E$, we have

$$
\left.\forall \mathbf{z} \in \mathbb{V}, \quad-\mathrm{a}_{1}(\mathbf{z}, \tilde{\mathbf{z}})=\left(\begin{array}{l}
\operatorname{div} \sigma_{F}(\widetilde{\mathbf{v}}, \widetilde{q})-(\nabla \mathbf{w})^{T} \widetilde{\mathbf{v}}+(\mathbf{w} \cdot \nabla) \widetilde{\mathbf{v}} \\
0 \\
\mathcal{M}_{0,0}^{-1}\left(\int_{\partial S_{s}}-\sigma_{F}(\widetilde{\mathbf{v}}, \widetilde{q}) \mathbf{n}_{s} \cdot \partial_{\theta_{j}} \boldsymbol{\Phi}\left(0,0, \gamma_{y}\right)\right)_{j=1,2}
\end{array}\right), \mathbf{z}\right)_{0}
$$

Then, we have $E \subset D\left(A_{1}^{*}\right)$.

To prove the reverse inclusion, the same arguments as in the proof of [11, Lemma 2.7] lead to the existence of $\widetilde{q} \in \mathrm{L}^{2}\left(\mathscr{F}_{s}\right)$ such that

$$
\begin{cases}\operatorname{div} \sigma_{F}(\widetilde{\mathbf{v}}, \widetilde{q}) & \in \mathbf{L}^{2}\left(\mathscr{F}_{s}\right) \\ \operatorname{div} \widetilde{\mathbf{v}}=0 & \text { in } \mathscr{F}_{s} \\ \widetilde{\mathbf{v}}=0 & \text { on } \Gamma_{\mathrm{D}} \\ \widetilde{\mathbf{v}}=\sum_{j} \widetilde{\omega}_{j} \partial_{\theta_{j}} \mathbf{\Phi}(0,0, .) & \text { on } \partial S_{s} \\ \sigma_{F}(\widetilde{\mathbf{v}}, \widetilde{q}) \mathbf{n}+(\mathbf{w} \cdot \mathbf{n}) \widetilde{\mathbf{v}}=0 & \text { on } \Gamma_{\mathrm{N}}\end{cases}
$$

Then $\sigma_{F}(\widetilde{\mathbf{v}}, \widetilde{q}) \mathbf{n}$ belongs to $\mathbf{H}^{1 / 2}\left(\Gamma_{\mathrm{N}}\right)$, this implies that $(\widetilde{\mathbf{v}}, \widetilde{q})$ belongs to $\mathbf{H}^{3 / 2+\varepsilon_{0}}\left(\mathscr{F}_{s}\right) \times \mathrm{H}^{1 / 2+\varepsilon_{0}}\left(\mathscr{F}_{s}\right)$. This regularity is a consequence of [20, Theorem 9.4.5] and the arguments used in the proof of [23, Theorem 2.5]. This ends the proof.

Lemma 2.10. The adjoint of $A$ with respect to $(.,)_{0}$ is given by

$$
D\left(A^{*}\right)=D\left(A_{1}^{*}\right),
$$

and

$$
A^{*}\left(\begin{array}{c}
\mathbf{v} \\
\theta_{1} \\
\theta_{2} \\
\omega_{1} \\
\omega_{2}
\end{array}\right)=\Pi_{\mathrm{H}}\left(\begin{array}{l}
\operatorname{div} \sigma_{F}(\mathbf{v}, q)+(\mathbf{w} \cdot \nabla) \mathbf{v}-(\nabla \mathbf{w})^{T} \mathbf{v} \\
\int_{\mathscr{F}_{s}}\left(\begin{array}{c}
\mathbf{L}_{1} \cdot \mathbf{v} \\
\mathbf{L}_{2} \cdot \mathbf{v}
\end{array}\right)+\left(\mathbf{L}_{k+4} \cdot\left(\begin{array}{c}
\omega_{1} \\
\omega_{2}
\end{array}\right)\right)_{k=1,2} \\
\mathcal{M}_{0,0}^{-1}\left[\int_{\mathscr{F}_{s}}\left(\begin{array}{c}
\mathbf{L}_{3} \cdot \mathbf{v} \\
\mathbf{L}_{4} \cdot \mathbf{v}
\end{array}\right)-\int_{\partial S_{s}}\left(\begin{array}{c}
\sigma_{F}(\mathbf{v}, q) \mathbf{n}_{s} \cdot \partial_{\theta_{1}} \mathbf{\Phi}\left(0,0, \gamma_{y}\right) \\
\sigma_{F}(\mathbf{v}, q) \mathbf{n}_{s} \cdot \partial_{\theta_{2}} \mathbf{\Phi}\left(0,0, \gamma_{y}\right)
\end{array}\right)+\left(\begin{array}{c}
\theta_{1} \\
\theta_{2}
\end{array}\right)\right]
\end{array}\right)
$$

Proof. We have $A-A_{1} \in \mathcal{L}(\mathbb{H})$, then $A^{*}-A_{1}^{*} \in \mathcal{L}(\mathbb{H})$ and $D\left(A^{*}\right)=D\left(A_{1}^{*}\right)$.

A computation of $(A \mathbf{z}, \tilde{\mathbf{z}})_{0}$ for $\mathbf{z} \in D(A)$ and $\tilde{\mathbf{z}} \in D\left(A^{*}\right)$ gives the explicit expression of $A^{*}$. 


\subsection{Construction of a feedback operator}

We define the control operator $B \in \mathcal{L}\left(\mathbb{R}^{2}, \mathbb{H}\right)$ by

$$
B \mathbf{h}=\Pi_{\mathbb{H}}\left(0,0,0, \mathcal{M}_{0,0}^{-1} \mathbf{h}\right) .
$$

The linear system (2.1) with no source terms $(\mathbf{f}=0, \mathbf{g}=0, \mathbf{s}=0)$ can be rewritten under the form

$$
\left\{\begin{array}{l}
\mathbf{z}^{\prime}(t)=A \mathbf{z}(t)+B \mathbf{h}(t), \quad t>0 \\
\mathbf{z}(0)=\mathbf{z}_{0}
\end{array}\right.
$$

where $\mathbf{z}=\left(\mathbf{v}, \theta_{1}, \theta_{2}, \dot{\theta}_{1}, \dot{\theta}_{2}\right)$ and $\mathbf{z}_{0}=\left(\mathbf{v}_{0}, \theta_{1,0}, \theta_{2,0}, \omega_{1,0}, \omega_{2,0}\right)$.

We want to exhibit a control $\mathbf{h}$ under a feedback form that stabilizes problem (2.20). In order to guarantee a decay rate $\delta>0$ of the solution to this problem, we consider the stabilization of $\mathbf{z}_{\delta}=e^{\delta t} \mathbf{z}$, solution to the problem

$$
\left\{\begin{array}{l}
\mathbf{z}_{\delta}^{\prime}(t)=(A+\delta \mathrm{I}) \mathbf{z}_{\delta}(t)+B \mathbf{h}_{\delta}(t), \quad t>0 \\
\mathbf{z}_{\delta}(0)=\mathbf{z}_{0}
\end{array}\right.
$$

where

$$
\mathbf{h}_{\delta}(t)=e^{\delta t} \mathbf{h}(t), \quad t>0 .
$$

Our goal is to find a control $\mathbf{h}$ providing the stabilization of (2.21).

Lemma 2.11. The adjoint operator of $B$ with respect to $(., .)_{0}$ is bounded, $B \in \mathcal{L}\left(\mathbb{H}, \mathbb{R}^{2}\right)$, and is given by

$$
B^{*}\left(\mathbf{v}, \theta_{1}, \theta_{2}, \omega_{1}, \omega_{2}\right)=\left(\begin{array}{l}
\omega_{1} \\
\omega_{2}
\end{array}\right)
$$

Proof. The operator $B$ is bounded, this is then a straightforward computation.

We define the unstable space associated to the operator $A+\delta$ I. Let $J_{u}$ be the set of eigenvalues $\lambda_{j}$ of $A$ such that $\operatorname{Re}\left(\lambda_{j}\right) \geq-\delta$. The set $J_{u}$ is exactly the set of all $\lambda \in \mathbb{C}$ such that $\lambda+\delta$ is an unstable eigenvalue of $A+\delta \mathrm{I}$. According to Lemmas 2.10 and 2.11 , Hypothesis $(\mathcal{H})_{\delta}$ can be rewritten as the following unique continuation property:

For every $\lambda \in J_{u}$ and all $\phi \in \mathbb{V}$ that obey $\left(A^{*}-\bar{\lambda} \mathrm{I}\right) \phi=0$ and $B^{*} \phi=0, \quad$ we have $\phi=0$,

where $\bar{\lambda}$ denotes the conjugate of $\lambda$.

The goal of this section is to construct a feedback control operator which uses only a finite number of scalar data to determine a control law that stabilizes problem (2.21), i.e.

Proposition 2.12. Let $\delta>0$ such that hypothesis $(\mathcal{H})_{\delta}$ is fulfilled. There exists $\mathcal{K}_{\delta} \in \mathcal{L}\left(\mathbf{L}^{2}\left(\mathscr{F}_{s}\right) \times \mathbb{R}^{4}, \mathbb{R}^{2}\right)$ such that the operator $A+\delta \mathrm{I}+B \mathcal{K}_{\delta}$ generates a stable analytic semigroup on $\mathbb{H}$.

Lemma 2.8 implies that the spectrum of the operator $A$ consists of isolated eigenvalues with finite algebraic multiplicities, moreover it has no finite cluster point. In addition, the operator $A$ generates an analytic semigroup, the control operator $B$ belongs to $\mathcal{L}\left(\mathbb{R}^{2}, \mathbb{H}\right)$ and we assume (2.22). Proposition 2.12 is then a consequence of the Fattorini criterion [4, Theorem 1.6].

In order to make the strategy clear, we provide a proof of Proposition 2.12.

Proof. We denote $\mathrm{G}\left(\lambda_{j}\right)$ the generalized eigenspace of $A$ associated to the eigenvalue $\lambda_{j}$ and we define the unstable space for $A+\delta \mathrm{I}$ by

$$
\mathbb{Z}_{u}=\bigoplus_{\lambda \in J_{u}} \mathrm{G}(\lambda)
$$

As $A+\delta$ I generates an analytic semigroup on $\mathbb{H}$ and has compact resolvent (see Lemma 2.8), $J_{u}$ is finite and every $\mathrm{G}\left(\lambda_{j}\right)$ is finite dimensional. Then the space $\mathbb{Z}_{u}$ is finite dimensional and we denote $d_{u}=\operatorname{dim}\left(\mathbb{Z}_{u}\right)$.

Besides, we can construct in the same way a space $\mathbb{Z}_{s}$ that contains all the stable eigenvectors of $A+\delta \mathrm{I}$, that is invariant under $\left(e^{t A}\right)_{t \geq 0}$ and such that

$$
\mathbb{H}=\mathbb{Z}_{u} \bigoplus \mathbb{Z}_{s}
$$

We denote $\Pi_{u}$ the projection of $\mathbb{H}$ onto $\mathbb{Z}_{u}$ along $\mathbb{Z}_{s}$ and $\Pi_{s}$ the projection of $\mathbb{H}$ onto $\mathbb{Z}_{s}$ along $\mathbb{Z}_{u}$. In the same way, we denote $\mathbb{Z}_{u}^{*}\left(\right.$ respectively $\mathbb{Z}_{s}^{*}$ ) the direct sum of the generalized eigenspaces of $A^{*}$ associated to an eigenvalue belonging (respectively not belonging) to $J_{u}$. 
According to [13, Lemma 6.2], there exist respectively two biorthogonal families $\left(\mathbf{z}_{i}\right)=\left(\mathbf{v}_{i}, \theta_{1, i}, \theta_{2, i}, \omega_{1, i}, \omega_{2, i}\right)$ and $\left(\tilde{\mathbf{z}}_{j}\right)=\left(\tilde{\mathbf{v}}_{j}, \tilde{\theta}_{1, j}, \tilde{\theta}_{2, j}, \tilde{\omega}_{1, j}, \tilde{\omega}_{2, j}\right)$ of $\mathbb{Z}_{u}$ and $\mathbb{Z}_{u}^{*}$ satisfying the condition

$$
\left(\mathbf{z}_{i}, \tilde{\mathbf{z}}_{j}\right)_{0}=\delta_{i j}
$$

and $\Pi_{u}\left(\mathbf{v}, \theta_{1}, \theta_{2}, \omega_{1}, \omega_{2}\right)=\sum_{i=1}^{d_{u}}\left(\left(\mathbf{v}, \theta_{1}, \theta_{2}, \omega_{1}, \omega_{2}\right),\left(\tilde{\mathbf{v}}_{i}, \tilde{\theta}_{1, i}, \tilde{\theta}_{2, i}, \tilde{\omega}_{1, i}, \tilde{\omega}_{2, i}\right)\right)_{0}\left(\mathbf{v}_{i}, \theta_{1, i}, \theta_{2, i}, \omega_{1, i}, \omega_{2, i}\right)$. We can also choose $\mathbf{z}_{i}$ and $\tilde{\mathbf{z}}_{j}$ to be respectively generalized eigenvectors of $A$ and $A^{*}$.

The adjoint $\Pi_{u}^{*}$ of $\Pi_{u}$ is the projection of $\mathbb{H}$ onto $\mathbb{Z}_{u}^{*}$ along $\mathbb{Z}_{s}^{*}$ as a straightforward calculation gives

$$
\forall \tilde{\mathbf{z}} \in \mathbb{H}, \Pi_{u}^{*} \tilde{\mathbf{z}}=\sum_{i=1}^{d_{u}}\left(\mathbf{z}_{i}, \tilde{\mathbf{z}}\right)_{0} \tilde{\mathbf{z}}_{i} .
$$

Denoting $\mathbf{z}_{u}=\Pi_{u} \mathbf{z}$ for every $\mathbf{z} \in \mathbb{H}$, the projection of (2.21) onto $\mathbb{Z}_{u}$ reads

$$
\left\{\begin{array}{l}
\mathbf{z}_{u}^{\prime}(t)=A_{u} \mathbf{z}_{u}(t)+\delta \mathbf{z}_{u}(t)+B_{u} \mathbf{h}_{u}(t) \quad t>0, \\
\mathbf{z}_{u}(0)=\Pi_{u} \mathbf{z}_{0},
\end{array}\right.
$$

where $A_{u}=\Pi_{u} A, B_{u}=\Pi_{u} B$ and $\mathbf{h}_{u}=\mathbf{h}_{\delta}$. Note that this formulation uses $A_{u}\left(\left(\mathrm{I}-\Pi_{u}\right) \mathbf{z}\right)=0$, which holds since $\mathbb{Z}_{s}$ is stable under $A+\delta \mathrm{I}$.

At this point, we use the following lemma that is proven just after the end of the current proof.

Lemma 2.13. Under Hypothesis $(\mathcal{H})_{\delta}$, problem $(2.24)$ is controllable on $\mathbb{Z}_{u}$.

Lemma 2.13 implies that problem (2.24) is stabilizable. Then the feedback control law

$$
\mathbf{h}_{u}=-B_{u}^{*} \mathcal{R}_{\delta} \mathbf{z}_{u}
$$

stabilizes problem (2.24), where $\mathcal{R}_{\delta} \in \mathcal{L}(\mathbb{H})$ is the unique positive self-adjoint solution in $\mathcal{L}(\mathbb{H})$ to the Riccati equation

$$
\mathcal{R}_{\delta}\left(A_{u}+\delta \mathrm{I}\right)+\left(A_{u}^{*}+\delta \mathrm{I}\right) \mathcal{R}_{\delta}+\mathrm{I}-\mathcal{R}_{\delta} B_{u} B_{u}^{*} \mathcal{R}_{\delta}=0,
$$

(for more information see [10, Section 6.2]).

Now we can construct a feedback law

$$
\tilde{\mathcal{K}}_{\delta}\left(\mathbf{z}_{\delta}\right)=\mathbf{h}_{u}=-B_{u}^{*} \mathcal{R}_{\delta} \Pi_{u} \mathbf{z}_{\delta}
$$

that stabilizes problem $(2.21)$. The feedback operator $\tilde{\mathcal{K}}_{\delta}$ belongs to $\mathcal{L}\left(\mathbb{H}, \mathbb{R}^{2}\right)$ and can be extended to an element $\mathcal{K}_{\delta}$ of $\mathcal{L}\left(\mathbf{L}^{2}\left(\mathscr{F}_{s}\right) \times \mathbb{R}^{4}, \mathbb{R}^{2}\right)$,

$$
\mathcal{K}_{\delta} \mathbf{z}_{\delta}=\tilde{\mathcal{K}}_{\delta} \Pi_{\mathbb{H}} \mathbf{z}_{\delta}=-B_{u}^{*} \mathcal{R}_{\delta} \Pi_{u} \Pi_{\mathbb{H}} \mathbf{z}_{\delta}, \text { for all } \mathbf{z}_{\delta} \in \mathbf{L}^{2}\left(\mathscr{F}_{s}\right) \times \mathbb{R}^{4}
$$

The operator $A_{u}+\delta \mathrm{I}+B_{u} \mathcal{K}_{\delta}$ generates an analytic stable semigroup on $\mathbb{Z}_{u}$.

Finally, let us prove that the operator $A+\delta \mathrm{I}+B \mathcal{K}_{\delta}$ generates an analytic stable semigroup on $\mathrm{H}$. We can use the decomposition $\mathbf{z}_{\delta}=\Pi_{u} \mathbf{z}_{\delta}+\Pi_{s} \mathbf{z}_{\delta}$, where $\Pi_{u} \mathbf{z}_{\delta}$ and $\Pi_{s} \mathbf{z}_{\delta}$ fulfil

$$
\begin{aligned}
& \begin{cases}\Pi_{u} \mathbf{z}_{\delta}^{\prime}=\left(A_{u}+\delta \mathrm{I}\right) \Pi_{u} \mathbf{z}_{\delta}+B_{u} \mathcal{K}_{\delta} \Pi_{u} \mathbf{z}_{\delta} & t>0, \\
\Pi_{u} \mathbf{z}_{\delta}(0)=\Pi_{u} \mathbf{z}_{0},\end{cases} \\
& \begin{cases}\Pi_{s} \mathbf{z}_{\delta}^{\prime}=\left(A_{s}+\delta \mathrm{I}\right) \Pi_{s} \mathbf{z}_{\delta}+B_{s} \mathcal{K}_{\delta} \Pi_{u} \mathbf{z}_{\delta} & t>0, \\
\Pi_{s} \mathbf{z}_{\delta}(0)=\Pi_{s} \mathbf{z}_{0},\end{cases}
\end{aligned}
$$

where $A_{s}=\Pi_{s} A, B_{s}=\Pi_{s} B$ and where we have used $\Pi_{s} A \Pi_{u}=\Pi_{u} A \Pi_{s}=0$.

As the operator $A_{u}+\delta \mathrm{I}+B \mathcal{K}_{\delta}$ generates an analytic stable semigroup on $\mathbb{Z}_{u}$, we have $\left\|\Pi_{u} \mathbf{z}_{\delta}\right\|_{\mathrm{L}^{2}(0, \infty ; \mathrm{H})} \leq C$. Moreover, according to the definition of $J_{u}$, the operator $A_{s}+\delta \mathrm{I}$ generates an analytic stable semigroup on $\mathbb{Z}_{s}$, then according to $\left[6\right.$, Theorem 3.1.(i), p. 143], we have $\left\|\Pi_{s} \mathbf{z}_{\delta}\right\|_{\mathrm{L}^{2}(0, \infty ; \mathbb{H})} \leq C$. Hence $A+\delta \mathrm{I}+B \mathcal{K}_{\delta}$ generates an analytic stable semigroup on $\mathrm{H}$. This ends the proof of Proposition 2.12.

Proof of Lemma 2.13. According to the Hautus test (see for instance [30, Proposition 1.5.1]), this result is equivalent to $\operatorname{Ker}\left(A_{u}^{*}+\delta \mathrm{I}-\bar{\lambda} \mathrm{I}\right) \cap \operatorname{Ker}\left(B_{u}^{*}\right)=\{0\}$ for every eigenvalue $\lambda$ of $A_{u}+\delta \mathrm{I}$, i.e. $\operatorname{Ker}\left(A_{u}^{*}-\bar{\lambda} \mathrm{I}\right) \cap \operatorname{Ker}\left(B_{u}^{*}\right)=\{0\}$ for every eigenvalue $\lambda$ of $A_{u}$.

The definition of $\mathbb{Z}_{u}$ implies that this space is stable under $A$. Hence, for every $\mathbf{z}_{u} \in \mathbb{Z}_{u}, A_{u} \mathbf{z}_{u}=A \mathbf{z}_{u}$. Then, the eigenvalues of $A_{u}$ are the elements of $J_{u}$.

Now, we need to compute $A_{u}^{*}$ and $B_{u}^{*}$, the adjoint operators of $A_{u}$ and $B_{u}$ with respect to $\mathbb{H}_{\text {. Let }} \mathbf{z}_{u}^{*} \in \mathbb{Z}_{u}^{*}$ and $\mathbf{z} \in \mathbb{H}$, we have

$$
\left(A_{u}^{*} \mathbf{z}_{u}^{*}, \mathbf{z}\right)_{0}=\left(\mathbf{z}_{u}^{*}, \Pi_{u} A \mathbf{z}\right)_{0}=\left(A^{*} \mathbf{z}_{u}^{*}, \mathbf{z}\right)_{0}
$$


and

$$
\left(B_{u}^{*} \mathbf{z}_{u}^{*}, \mathbf{z}\right)_{0}=\left(\mathbf{z}_{u}^{*}, \Pi_{u} B \mathbf{z}\right)_{0}=\left(B^{*} \mathbf{z}_{u}^{*}, \mathbf{z}\right)_{0},
$$

then $\forall \mathbf{z}_{u}^{*} \in \mathbb{Z}_{u}^{*}$, we have $A_{u}^{*} \mathbf{z}_{u}^{*}=A^{*} \mathbf{z}_{u}^{*}$ and $B_{u}^{*} \mathbf{z}_{u}^{*}=B^{*} \mathbf{z}_{u}^{*}$.

Let us now prove the Hautus test on $\left(A_{u}^{*}, B_{u}^{*}\right)$. Let $\lambda \in \mathbb{C}$ be an eigenvalue of $A_{u}$, then $\lambda \in J_{u}$. Let $\mathbf{z}_{u}^{*} \in \mathbb{Z}_{u}^{*}$ and assume that $\mathbf{z}_{u}^{*} \in \operatorname{Ker}\left(A_{u}^{*}-\bar{\lambda} \mathrm{I}\right) \cap \operatorname{Ker}\left(B_{u}^{*}\right)$. We have $\left(A^{*}-\bar{\lambda} \mathrm{I}\right) \mathbf{z}_{u}^{*}=0$ and $B^{*} \mathbf{z}_{u}^{*}=0$. Thus, $\mathbf{z}_{u}^{*} \in D\left(A^{*}\right)$ and the property (2.22) implies that $\mathbf{z}_{u}^{*}=0$ and this ends the proof.

A consequence of Proposition 2.12 is the following lemma.

Lemma 2.14. Let $\mathbf{z}_{0} \in \mathbb{V}$ and $\mathbf{F} \in\left\{\mathbf{f}\right.$ with $\left.e^{\delta t} \mathbf{f} \in \mathrm{L}^{2}(0, \infty ; \mathbb{H})\right\}$. The solution $\mathbf{z}$ to the closed-loop problem

$$
\left\{\begin{array}{l}
\mathbf{z}^{\prime}=A \mathbf{z}+B \mathcal{K}_{\delta} \mathbf{z}+\mathbf{F} \quad t>0, \\
\mathbf{z}(0)=\mathbf{z}_{0}
\end{array}\right.
$$

belongs to $\left\{\mathbf{z}\right.$ with $\left.e^{\delta t} \mathbf{z} \in \mathrm{L}^{2}(0, \infty ; D(A)) \cap \mathrm{H}^{1}(0, \infty ; \mathbb{H}) \cap \mathscr{C}^{0}([0, \infty) ; \mathbb{V})\right\}$. Moreover, we have the estimate

$$
\left\|e^{\delta t} \mathbf{z}\right\|_{\mathrm{L}^{2}(0, \infty ; D(A)) \cap \mathrm{H}^{1}(0, \infty ; \mathbb{H}) \cap \mathscr{C}^{0}([0, \infty) ; \mathrm{V})} \leq C\left(\left\|\mathbf{z}_{0}\right\|_{\mathrm{V}}+\left\|e^{\delta t} \mathbf{F}\right\|_{\mathrm{L}^{2}(0, \infty ; \mathbb{H})}\right) .
$$

Proof. We have $\delta \mathrm{I}+B \mathcal{K}_{\delta} \in \mathcal{L}(\mathbb{H})$, then $D\left(A+\delta \mathrm{I}+B \mathcal{K}_{\delta}\right)=D(A)$ and by interpolation for $\lambda>0$ large enough, $D\left(\left(\lambda \mathrm{I}-A-\delta \mathrm{I}-B \mathcal{K}_{\delta}\right)^{1 / 2}\right)=D\left((\lambda \mathrm{I}-A)^{1 / 2}\right)=\mathbb{V}$, for the definition of these spaces see (2.14)-(2.15). Moreover, $e^{\delta t} \mathbf{F} \in \mathrm{L}^{2}(0, \infty ; \mathbb{H})$.

In addition, according to Proposition $2.12, A+\delta \mathrm{I}+B \mathcal{K}_{\delta}$ generates an analytic semigroup that is stable on $\mathbb{H}$, hence according to [6, Theorem 3.1.(i), p. 143], there exists $\mathbf{z}_{\delta} \in \mathrm{H}^{1}(0,+\infty ; \mathbb{H}) \cap \mathrm{L}^{2}(0,+\infty ; D(A))$ satisfying

$$
\left\{\begin{array}{l}
\mathbf{z}_{\delta}^{\prime}=\left(A+\delta \mathrm{I}+B \mathcal{K}_{\delta}\right) \mathbf{z}_{\delta}+e^{\delta t} \mathbf{F} \quad t>0 \\
\mathbf{z}_{\delta}(0)=\mathbf{z}_{0}
\end{array}\right.
$$

By interpolation, $\mathbf{z}_{\delta}$ also belongs to $\mathscr{C}^{0}([0, \infty) ; \mathbb{V})$. Now, $\mathbf{z}=e^{-\delta t} \mathbf{z}_{\delta}$ belongs to $\left\{\mathbf{z}\right.$ with $e^{\delta t} \mathbf{z} \in \mathrm{L}^{2}(0, \infty ; D(A)) \cap$ $\left.\mathrm{H}^{1}(0, \infty ; \mathbb{H}) \cap \mathscr{C}^{0}([0, \infty) ; \mathbb{V})\right\}$ and is solution to $(2.26)$. Moreover, we have the estimate $(2.27)$ as a consequence of [6, Theorem 3.1.(i), p. 143].

\subsection{Stabilization of the linear system $(2.1)$}

In this section we prove that the feedback operator $\mathcal{K}_{\delta}$ stabilizes the linear problem (2.1). The velocity is decomposed as $\mathbf{v}=\widetilde{\mathbf{v}}+\mathbf{v}_{\mathbf{g}}$ where $\mathbf{v}_{\mathbf{g}}$ is a lifting of $\mathbf{g}$. We first prove this result for distributed source terms only (i.e. on $\widetilde{\mathbf{v}}$ ) and then for boundary source terms (i.e. for $\widetilde{\mathbf{v}}+\mathbf{v}_{\mathbf{g}}$ ). The first part is a consequence of the study of the semigroup.

\subsubsection{Stabilization of the problem with nonhomogeneous distributed source terms}

In this section we prove a stabilization result for the following system that corresponds to (2.1) with $\mathbf{g}=0$,

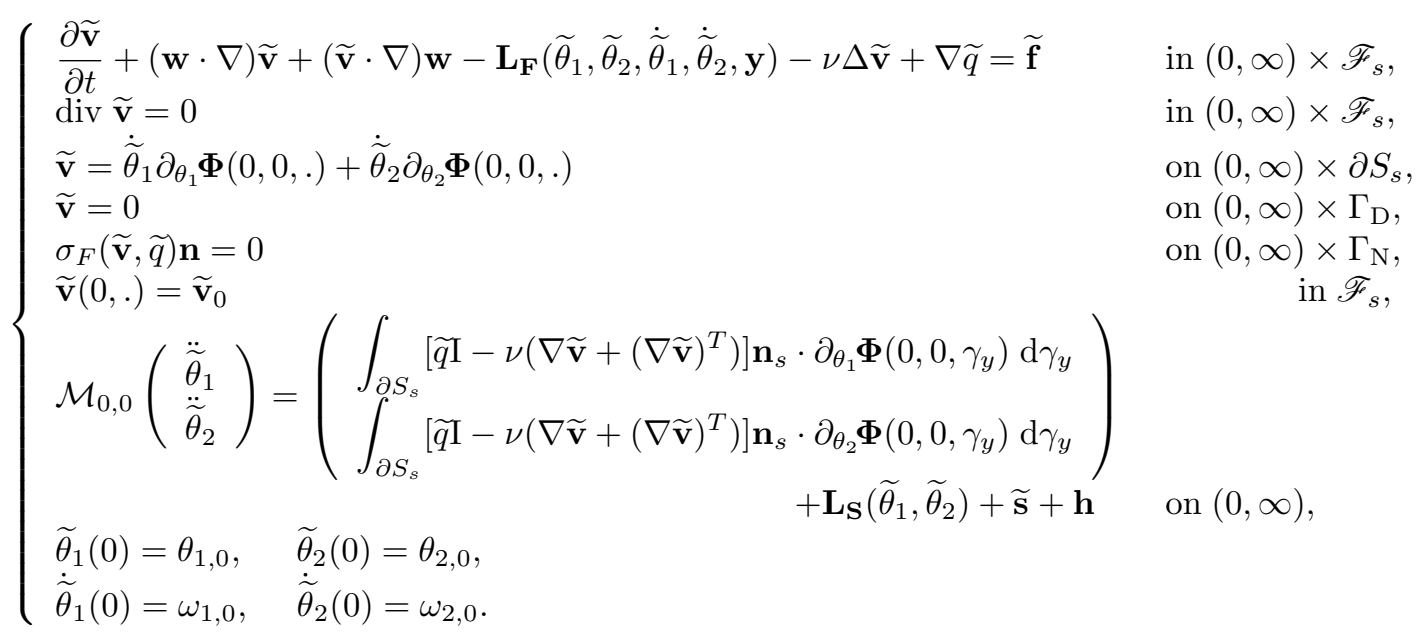

Proposition 2.15. Let $\delta>0$ and let $(\mathcal{H})_{\delta}$ be fulfilled. For every $\left(\widetilde{\mathbf{v}}_{0}, \theta_{1,0}, \theta_{2,0}, \omega_{1,0}, \omega_{2,0}\right) \in \mathbf{H}^{1}\left(\mathscr{F}_{s}\right) \times \mathbb{R}^{4}$, fulfilling the compatibility conditions

$$
\begin{cases}\operatorname{div} \widetilde{\mathbf{v}}_{0}=0 & \text { in } \mathscr{F}_{s} \\ \widetilde{\mathbf{v}}_{0}=\sum_{j=1}^{2} \omega_{j, 0} \partial_{\theta_{j}} \mathbf{\Phi}(0,0, .) & \text { on } \partial S_{s} \\ \widetilde{\mathbf{v}}_{0}=0 & \text { on } \Gamma_{\mathrm{D}}\end{cases}
$$


and every source terms $\widetilde{\mathbf{f}} \in \mathbb{F}_{\delta}^{\infty}$ and $\widetilde{\mathbf{s}} \in \mathbb{S}_{\delta}^{\infty}$ problem (2.28) with the control taken as $\mathbf{h}=\mathcal{K}_{\delta}\left(\widetilde{\mathbf{v}}, \widetilde{\theta}_{1}, \widetilde{\theta}_{2}, \dot{\widetilde{\theta}}_{1}, \dot{\tilde{\theta}}_{2}\right)$ admits a unique solution $\left(\widetilde{\mathbf{v}}, \widetilde{q}, \widetilde{\theta}_{1}, \widetilde{\theta}_{2}\right) \in \mathbb{U}_{\delta}^{\infty} \times \mathbb{P}_{\delta}^{\infty} \times \Theta_{\delta}^{\infty}$ with the following estimate

$$
\|\widetilde{\mathbf{v}}\|_{\mathbb{U}_{\delta}^{\infty}}+\|\widetilde{q}\|_{\mathbb{P}_{\delta}^{\infty}}+\left\|\left(\widetilde{\theta}_{1}, \widetilde{\theta}_{2}\right)\right\|_{\Theta_{\delta}^{\infty}} \leq C\left(\left\|\widetilde{\mathbf{v}}_{0}\right\|_{\mathbf{H}^{1}\left(\mathscr{F}_{s}\right)}+\left|\theta_{1,0}\right|+\left|\theta_{2,0}\right|+\left|\omega_{1,0}\right|+\left|\omega_{2,0}\right|+\|\widetilde{\mathbf{f}}\|_{\mathbb{F}_{\delta}^{\infty}}+\|\widetilde{\mathbf{S}}\|_{\mathbb{S}_{\delta}^{\infty}}\right),
$$

where $C$ does not depend on the initial data and on the source terms.

Proof. The initial data $\left(\widetilde{\mathbf{v}}_{0}, \theta_{1,0}, \theta_{2,0}, \omega_{1,0}, \omega_{2,0}\right)$ fulfil the compatibility conditions $(2.29)$, that is why $\mathbf{z}_{0}=$ $\left(\widetilde{\mathbf{v}}_{0}, \theta_{1,0}, \theta_{2,0}, \omega_{1,0}, \omega_{2,0}\right)$ belongs to $\mathbb{V}$. Moreover, as $\widetilde{\mathbf{f}} \in \mathbb{F}_{\delta}^{\infty}$ and $\widetilde{\mathbf{s}} \in \mathbb{S}_{\delta}^{\infty}$,

$$
\mathbf{F}=\left(\begin{array}{l}
\widetilde{\mathbf{f}} \\
0 \\
0 \\
\mathcal{M}_{0,0}^{-1} \widetilde{\mathbf{s}}
\end{array}\right)
$$

belongs to $\left\{\mathbf{f}\right.$ with $\left.e^{\delta t} \mathbf{f} \in \mathrm{L}^{2}(0, \infty ; \mathbb{H})\right\}$. Then, according to Lemma 2.14, the solution to (2.26) belongs to $\left\{\mathbf{z}\right.$ with $\left.e^{\delta t} \mathbf{z} \in \mathrm{L}^{2}(0, \infty ; D(A)) \cap \mathrm{H}^{1}(0, \infty ; \mathbb{H}) \cap \mathscr{C}^{0}([0, \infty) ; \mathbb{V})\right\}$.

Now, we use the identity $\mathbf{z}=\left(\widetilde{\mathbf{v}}, \widetilde{\theta}_{1}, \widetilde{\theta}_{2}, \widetilde{\omega}_{1}, \widetilde{\omega}_{2}\right)$. According to Lemmas 2.2 and $2.6, \widetilde{\mathbf{v}} \in \mathbb{U}_{\delta}^{\infty},\left(\widetilde{\theta}_{1}, \widetilde{\theta}_{2}\right) \in \Theta_{\delta}^{\infty}$ and there exists $\widetilde{q} \in \mathbb{P}_{\delta}^{\infty}$ such that problem (2.26) reads

$$
\frac{\mathrm{d}}{\mathrm{d} t}\left(\begin{array}{c}
\widetilde{\mathbf{v}} \\
\widetilde{\theta}_{1} \\
\widetilde{\theta}_{2} \\
\widetilde{\omega}_{1} \\
\widetilde{\omega}_{2}
\end{array}\right)=\left(\begin{array}{l}
\operatorname{div} \sigma_{F}(\widetilde{\mathbf{v}}, \widetilde{q})+\mathbf{L}_{\mathbf{F}}\left(\widetilde{\theta}_{1}, \widetilde{\theta}_{2}, \widetilde{\omega}_{1}, \widetilde{\omega}_{2}, \mathbf{y}\right)-(\widetilde{\mathbf{v}} \cdot \nabla) \mathbf{w}-(\mathbf{w} \cdot \nabla) \widetilde{\mathbf{v}}+\widetilde{\mathbf{f}} \\
\widetilde{\omega}_{1} \\
\widetilde{\omega}_{2} \\
\mathcal{M}_{0,0}^{-1}\left(\left(\begin{array}{l}
\int_{\partial S_{s}}-\sigma_{F}(\widetilde{\mathbf{v}}, \widetilde{q}) \mathbf{n}_{s} \cdot \partial_{\theta_{1}} \mathbf{\Phi}\left(0,0, \gamma_{y}\right) \\
\int_{\partial S_{s}}^{-}-\sigma_{F}(\widetilde{\mathbf{v}}, \widetilde{q}) \mathbf{n}_{s} \cdot \partial_{\theta_{2}} \mathbf{\Phi}\left(0,0, \gamma_{y}\right)
\end{array}\right)+\mathbf{L}_{\mathbf{S}}\left(\widetilde{\theta}_{1}, \widetilde{\theta}_{2}\right)+\mathcal{K}_{\delta}\left(\widetilde{\mathbf{v}}, \widetilde{\theta}_{1}, \widetilde{\theta}_{2}, \widetilde{\omega}_{1}, \widetilde{\omega}_{2}\right)+\widetilde{\mathbf{s}}\right)
\end{array}\right),
$$

where $\mathbf{L}_{\mathbf{F}}$ and $\mathbf{L}_{\mathbf{S}}$ are defined in $(2.2)-(2.3)$. Then, $\left(\widetilde{\mathbf{v}}, \widetilde{q}, \widetilde{\theta}_{1}, \widetilde{\theta}_{2}\right)$ is solution to (2.28).

Finally, the estimate $(2.30)$ is a consequence of $(2.27)$.

\subsubsection{A first stabilization result for the problem with a nonhomogeneous boundary datum}

In the sequel, for $\mathbf{g} \in \mathbb{G}_{\delta}^{\infty}$ we consider $\mathbf{v}_{\mathbf{g}} \in\left\{\mathbf{v}\right.$ with $\left.e^{\delta t} \mathbf{v} \in \mathrm{H}^{1}\left(0, \infty ; \mathbf{H}^{2}\left(\mathscr{F}_{s}\right)\right)\right\}$ that satisfies

$$
\begin{cases}\mathbf{v}_{\mathbf{g}}=\mathbf{g} & \text { on }(0, \infty) \times \partial S_{s} \\ \operatorname{div} \mathbf{v}_{\mathbf{g}}=0 & \text { in }(0, \infty) \times \mathscr{F}_{s} \\ \mathbf{v}_{\mathbf{g}}=0 & \text { on }(0, \infty) \times \Gamma_{\mathrm{D}} \\ \left(\nabla \mathbf{v}_{\mathbf{g}}+\left(\nabla \mathbf{v}_{\mathbf{g}}\right)^{T}\right) \mathbf{n}=0 & \text { on }(0, \infty) \times \Gamma_{\mathrm{N}}\end{cases}
$$

and

$$
\left\|\mathbf{v}_{\mathbf{g}} e^{\delta t}\right\|_{\mathrm{H}^{1}\left(0, \infty ; \mathbf{H}^{2}\left(\mathscr{F}_{s}\right)\right)} \leq C\|\mathbf{g}\|_{\mathrm{G}_{\delta}^{\infty}}
$$

see [23, Theorem 2.16] for a proof of the existence of $\mathbf{v}_{\mathbf{g}}$.

The following proposition enables the stabilization of problem (2.1) with $\mathbf{g} \neq 0$. However, contrary to Proposition 2.1, the feedback control is $\mathcal{K}_{\delta}\left(\mathbf{v}-\mathbf{v}_{\mathbf{g}}, \theta_{1}, \theta_{2}, \dot{\theta}_{1}, \dot{\theta}_{2}\right)$ instead of $\mathcal{K}_{\delta}\left(\mathbf{v}, \theta_{1}, \theta_{2}, \dot{\theta}_{1}, \dot{\theta}_{2}\right)$.

Proposition 2.16. Let $\delta>0$ and let $(\mathcal{H})_{\delta}$ be fulfilled. For every $\left(\mathbf{v}_{0}, \theta_{1,0}, \theta_{2,0}, \omega_{1,0}, \omega_{2,0}\right) \in \mathbf{H}^{1}\left(\mathscr{F}_{s}\right) \times \mathbb{R}^{4}$, $\mathbf{f} \in \mathbb{F}_{\delta}^{\infty}, \mathbf{g} \in \mathbb{G}_{\delta}^{\infty}$ and $\mathbf{s} \in \mathbb{S}_{\delta}^{\infty}$ fulfilling the compatibility conditions (2.10), problem (2.1) with the control $\mathbf{h}=\mathcal{K}_{\delta}\left(\mathbf{v}-\mathbf{v}_{\mathbf{g}}, \theta_{1}, \theta_{2}, \dot{\theta}_{1}, \dot{\theta}_{2}\right)$ admits a unique solution $\left(\mathbf{v}, q, \theta_{1}, \theta_{2}\right) \in \mathbb{U}_{\delta}^{\infty} \times \mathbb{P}_{\delta}^{\infty} \times \Theta_{\delta}^{\infty}$ with the following estimate,

$$
\|\mathbf{v}\|_{\mathbb{U}_{\delta}^{\infty}}+\|q\|_{\mathbb{P}_{\delta}^{\infty}}+\left\|\left(\theta_{1}, \theta_{2}\right)\right\|_{\Theta_{\delta}^{\infty}} \leq C\left(\left\|\mathbf{v}_{0}\right\|_{\mathbf{H}^{1}\left(\mathscr{F}_{s}\right)}+\left|\theta_{1,0}\right|+\left|\theta_{2,0}\right|+\left|\omega_{1,0}\right|+\left|\omega_{2,0}\right|+\|\mathbf{f}\|_{\mathrm{F}_{\delta}^{\infty}}+\|\mathbf{g}\|_{\mathbb{G}_{\delta}^{\infty}}+\|\mathbf{s}\|_{\mathbb{S}_{\delta}^{\infty}}\right)
$$

where $C$ does not depend on the initial conditions and the source terms.

Proof. Let $\left(\mathbf{v}, q, \theta_{1}, \theta_{2}\right)$ be the solution to $(2.1)$ with $\mathbf{h}=\mathcal{K}_{\delta}\left(\mathbf{v}-\mathbf{v}_{\mathbf{g}}, \theta_{1}, \theta_{2}, \dot{\theta}_{1}, \dot{\theta}_{2}\right)$. We now consider $\left(\widetilde{\mathbf{v}}, \widetilde{q}, \widetilde{\theta}_{1}, \widetilde{\theta}_{2}\right)=$ $\left(\mathbf{v}-\mathbf{v}_{\mathbf{g}}, q, \theta_{1}, \theta_{2}\right)$, it is solution to problem (2.28) where

$$
\begin{aligned}
& \widetilde{\mathbf{v}}_{0}=\mathbf{v}_{0}-\mathbf{v}_{\mathbf{g}}(0), \\
& \widetilde{\mathbf{f}}=\mathbf{f}-\partial_{t} \mathbf{v}_{\mathbf{g}}-(\mathbf{w} \cdot \nabla) \mathbf{v}_{\mathbf{g}}-\left(\mathbf{v}_{\mathbf{g}} \cdot \nabla\right) \mathbf{w}+\nu \Delta \mathbf{v}_{\mathbf{g}}, \\
& \widetilde{\mathbf{s}}=\mathbf{s}-\left(\int_{\partial S_{s}} \nu\left(\nabla \mathbf{v}_{\mathbf{g}}+\nabla \mathbf{v}_{\mathbf{g}}^{T}\right) \mathbf{n}_{s} \cdot \partial_{\theta_{j}} \mathbf{\Phi}\left(0,0, \gamma_{y}\right)\right)_{j=1,2} .
\end{aligned}
$$


The initial data $\left(\widetilde{\mathbf{v}}_{0}, \theta_{1,0}, \theta_{2,0}, \omega_{1,0}, \omega_{2,0}\right)$ fulfil $(2.29)$, then all the terms have the good regularity and we have the estimate (2.30). We now use (2.32) and get

$$
\begin{aligned}
& \|\widetilde{\mathbf{f}}\|_{\mathbb{F}_{\delta}^{\infty}} \leq\|\mathbf{f}\|_{\mathbb{F}_{\delta}^{\infty}}+C\|\mathbf{g}\|_{\mathbb{G}_{\delta}^{\infty}}, \\
& \|\widetilde{\mathbf{s}}\|_{\mathbb{S}_{\delta}^{\infty}} \leq\|\mathbf{s}\|_{\mathbb{S}_{\delta}^{\infty}}+C\|\mathbf{g}\|_{\mathbb{G}_{\delta}^{\infty}} .
\end{aligned}
$$

All these estimates prove the estimate (2.33).

\subsubsection{Proof of Proposition 2.1}

Proof. In Proposition 2.16, we have proven that the control $\mathbf{h}=\mathcal{K}_{\delta}\left(\mathbf{v}-\mathbf{v}_{\mathbf{g}}, \theta_{1}, \theta_{2}, \dot{\theta}_{1}, \dot{\theta}_{2}\right)$ stabilizes the problem (2.1). We now want to prove that the control $\mathbf{h}=\mathcal{K}_{\delta}\left(\mathbf{v}, \theta_{1}, \theta_{2}, \dot{\theta}_{1}, \dot{\theta}_{2}\right)$ also stabilizes the same problem.

Let $\left(\mathbf{v}, q, \theta_{1}, \theta_{2}\right)$ be the solution to (2.1) with $\mathbf{h}=\mathcal{K}_{\delta}\left(\mathbf{v}-\mathbf{v}_{\mathbf{g}}, \theta_{1}, \theta_{2}, \dot{\theta}_{1}, \dot{\theta}_{2}\right)$. According to Proposition 2.16, we have the estimate $(2.33)$.

Let $\left(\widehat{\mathbf{v}}, \widehat{q}, \widehat{\theta}_{1}, \widehat{\theta}_{2}\right)$ be the solution to $(2.1)$ with $\mathbf{h}=\mathcal{K}_{\delta}\left(\widehat{\mathbf{v}}, \widehat{\theta}_{1}, \widehat{\theta}_{2}, \dot{\hat{\theta}}_{1}, \dot{\hat{\theta}}_{2}\right)$. We now consider

$$
\left(\widetilde{\mathbf{v}}, \widetilde{q}, \widetilde{\theta}_{1}, \widetilde{\theta}_{2}\right)=\left(\mathbf{v}, q, \theta_{1}, \theta_{2}\right)-\left(\widehat{\mathbf{v}}, \widehat{q}, \widehat{\theta}_{1}, \widehat{\theta}_{2}\right),
$$

it is solution to $(2.28)$ with $\mathbf{f}=0, \mathbf{v}_{0}=0, \mathbf{s}=-\mathcal{K}_{\delta}\left(\mathbf{v}_{\mathbf{g}}, 0,0,0,0\right), \theta_{1,0}=0, \theta_{2,0}=0, \omega_{1,0}=0, \omega_{2,0}=0$ and $\mathbf{h}=\mathcal{K}_{\delta}\left(\widetilde{\mathbf{v}}, \widetilde{\theta}_{1}, \widetilde{\theta}_{2}, \dot{\widetilde{\theta}}_{1}, \dot{\widetilde{\theta}}_{2}\right)$. The initial data $(0,0,0,0,0)$ fulfil the compatibility conditions $(2.29)$, hence we have the estimate (2.30). When combining the estimates (2.30) and (2.33), we get

$$
\begin{aligned}
\|\widehat{\mathbf{v}}\|_{U_{\delta}^{\infty}}+\|\widehat{q}\|_{\mathbb{P}_{\delta}^{\infty}}+\left\|\left(\widehat{\theta}_{1}, \widehat{\theta}_{2}\right)\right\|_{\Theta_{\delta}^{\infty}} & \leq\|\mathbf{v}\|_{U_{\delta}^{\infty}}+\|\widetilde{\mathbf{v}}\|_{U_{\delta}^{\infty}}+\|q\|_{\mathbb{P}_{\delta}^{\infty}}+\|\widetilde{q}\|_{\mathbb{P}_{\delta}^{\infty}}+\left\|\left(\theta_{1}, \theta_{2}\right)\right\|_{\Theta_{\delta}^{\infty}}+\left\|\left(\widetilde{\theta}_{1}, \widetilde{\theta}_{2}\right)\right\|_{\Theta_{\delta}^{\infty}} \\
& \leq C\left(\left\|\mathbf{v}_{0}\right\|_{\mathbf{H}^{1}\left(\mathscr{F}_{s}\right)}+\left|\theta_{1,0}\right|+\left|\theta_{2,0}\right|+\left|\omega_{1,0}\right|+\left|\omega_{2,0}\right|+\|\mathbf{f}\|_{\mathbb{F}_{\delta}^{\infty}}+\|\mathbf{g}\|_{G_{\delta}^{\infty}}+\|\mathbf{s}\|_{S_{\delta}^{\infty}}\right) .
\end{aligned}
$$

This ends the proof of Proposition 2.1.

\section{Stabilization of the nonlinear closed loop system}

The proof of Theorem 1.6 will be developed in this section. As in Section 2 we consider $\left(\mathbf{f}_{\mathscr{F}}, \mathbf{u}^{i}, \mathbf{f}_{\mathbf{s}}\right) \in \mathbf{W}^{1, \infty}(\Omega) \times$ $\mathbf{U}^{i} \times \mathbb{R}^{2}$ and a stationary state $\left(\mathbf{w}, p_{\mathbf{w}}\right) \in \mathbf{H}_{\beta}^{2}\left(\mathscr{F}_{s}\right) \times \mathrm{H}_{\beta}^{1}\left(\mathscr{F}_{s}\right)$ that fulfil (1.17) (see Remark 1.9).

\subsection{The nonlinear problem in a fixed domain}

In this section, we are interested in writing the equations fulfilled by the difference between the solution to (1.15) and the stationary state. In order to do so, we consider the change of variables

$$
\forall \mathbf{y} \in \mathscr{F}_{s}, \quad \forall t \in(0, \infty), \quad\left\{\begin{array}{l}
\mathbf{v}(t, \mathbf{y})=\operatorname{cof}\left(\mathcal{J}_{\boldsymbol{\Phi}}\left(\theta_{1}(t), \theta_{2}(t), \mathbf{y}\right)\right)^{T} \mathbf{u}\left(t, \mathbf{\Phi}\left(\theta_{1}(t), \theta_{2}(t), \mathbf{y}\right)\right)-\mathbf{w}(\mathbf{y}), \\
q(t, \mathbf{y})=p\left(t, \boldsymbol{\Phi}\left(\theta_{1}(t), \theta_{2}(t), \mathbf{y}\right)\right)-p_{\mathbf{w}}(\mathbf{y}),
\end{array}\right.
$$

where $\boldsymbol{\Phi}$ is the diffeomorphism defined in (1.19) fulfilling (1.21), moreover $\mathcal{J}_{\mathbf{\Phi}}\left(\theta_{1}, \theta_{2}, \mathbf{y}\right)=\nabla_{\mathbf{y}} \mathbf{\Phi}\left(\theta_{1}, \theta_{2}, \mathbf{y}\right)$ and $\operatorname{cof}\left(\mathcal{J}_{\boldsymbol{\Phi}}\right)$ is the cofactor matrix of $\mathcal{J}_{\boldsymbol{\Phi}}$. This change of variables has been chosen in order to have a divergence free velocity $\mathbf{v}$ in the fixed domain.

One can show that under the feedback control $\mathbf{h}=\mathcal{K}_{\delta}\left(\mathbf{v}, \theta_{1}, \theta_{2}, \dot{\theta}_{1}, \dot{\theta}_{2}\right)$ defined in Proposition $2.1,\left(\mathbf{v}, q, \theta_{1}, \theta_{2}\right)$ is solution to the closed loop system

$$
\left\{\begin{array}{lr}
\frac{\partial \mathbf{v}}{\partial t}+(\mathbf{w} \cdot \nabla) \mathbf{v}+(\mathbf{v} \cdot \nabla) \mathbf{w}-\mathbf{L}_{\mathbf{F}}\left(\theta_{1}, \theta_{2}, \dot{\theta}_{1}, \dot{\theta}_{2}, \mathbf{y}\right)-\nu \Delta \mathbf{v}+\nabla q=\mathbf{f} & \text { in }(0, \infty) \times \mathscr{F}_{s}, \\
\operatorname{div} \mathbf{v}=0 & \text { in }(0, \infty) \times \mathscr{F}_{s}, \\
\mathbf{v}=\dot{\theta}_{1} \partial_{\theta_{1}} \mathbf{\Phi}\left(0,0, \gamma_{y}\right)+\dot{\theta}_{2} \partial_{\theta_{2}} \mathbf{\Phi}\left(0,0, \gamma_{y}\right)+\mathbf{g} & \text { on }(0, \infty) \times \partial S_{s}, \\
\mathbf{v}=0 & \text { on }(0, \infty) \times \Gamma_{\mathrm{D}}, \\
\sigma_{F}(\mathbf{v}, q) \mathbf{n}=0 & \text { on }(0, \infty) \times \Gamma_{\mathrm{N}}, \\
\mathbf{v}(0, \mathbf{y})=\mathbf{v}_{0}(\mathbf{y})=\operatorname{cof}\left(\mathcal{J}_{\mathbf{\Phi}}\left(\theta_{1,0}, \theta_{2,0}, \mathbf{y}\right)\right)^{T} \mathbf{u}_{0}\left(\mathbf{\Phi}\left(\theta_{1,0}, \theta_{2,0}, \mathbf{y}\right)\right)-\mathbf{w}(\mathbf{y}) & \text { in } \mathscr{F}_{s}, \\
\mathcal{M}_{0,0}\left(\begin{array}{c}
\ddot{\theta}_{1} \\
\ddot{\theta}_{2}
\end{array}\right)=\left(\begin{array}{l}
\int_{\partial S_{s}}-\sigma_{F}(\mathbf{v}, q) \mathbf{n}_{s} \cdot \partial_{\theta_{1}} \mathbf{\Phi}\left(0,0, \gamma_{y}\right) \\
\int_{\partial S_{s}}-\sigma_{F}(\mathbf{v}, q) \mathbf{n}_{s} \cdot \partial_{\theta_{2}} \mathbf{\Phi}\left(0,0, \gamma_{y}\right)
\end{array}\right) & \text { on }(0, \infty), \\
\left.\theta_{1}(0)=\mathbf{L}_{\mathbf{S}}, \quad \theta_{1}, \theta_{2}\right)+\mathbf{s}+\mathcal{K}_{\delta}\left(\mathbf{v}, \theta_{1}, \theta_{2}, \dot{\theta}_{1}, \dot{\theta}_{2}\right) & \\
\dot{\theta}_{1}(0)=\omega_{1,0}, \quad \dot{\theta}_{2}(0)=\theta_{2,0}, &
\end{array}\right.
$$


where the linear terms are

$$
\begin{aligned}
\mathbf{L}_{\mathbf{F}}\left(\theta_{1}, \theta_{2}, \dot{\theta}_{1}, \dot{\theta}_{2}, \mathbf{y}\right) & =\mathbf{L}_{1}(\mathbf{y}) \theta_{1}+\mathbf{L}_{2}(\mathbf{y}) \theta_{2}+\mathbf{L}_{3}(\mathbf{y}) \dot{\theta}_{1}+\mathbf{L}_{4}(\mathbf{y}) \dot{\theta}_{2}, \quad \forall \mathbf{y} \in \mathscr{F}_{s} \\
\mathbf{L}_{\mathbf{S}}\left(\theta_{1}, \theta_{2}\right) & =\mathbf{L}_{5} \theta_{1}+\mathbf{L}_{6} \theta_{2}
\end{aligned}
$$

and the coefficients $\mathbf{L}_{1}-\mathbf{L}_{6}$ are defined in Appendix A. Moreover $\mathcal{K}_{\delta}$ is the feedback operator given in (2.25) and the source terms are given by the nonlinear (at least quadratic) terms

$$
\left\{\begin{array}{l}
\mathbf{f}=\mathbf{f}^{N L}\left(\theta_{1}, \theta_{2}, \mathbf{v}, q\right) \\
\mathbf{g}=\mathbf{g}^{N L}\left(\theta_{1}, \theta_{2}\right) \\
\mathbf{s}=\mathbf{s}^{N L}\left(\theta_{1}, \theta_{2}, \mathbf{v}, q\right)
\end{array}\right.
$$

defined below

$$
\left\{\begin{array}{l}
\mathbf{f}^{N L}\left(\theta_{1}, \theta_{2}, \mathbf{v}, q\right)=\mathbf{F}\left(\theta_{1}, \theta_{2}, \mathbf{w}+\mathbf{v}, p_{\mathbf{w}}+q\right)+(\mathbf{w} \cdot \nabla) \mathbf{w}+(\mathbf{w} \cdot \nabla) \mathbf{v}+(\mathbf{v} \cdot \nabla) \mathbf{w}-\mathbf{L}_{\mathbf{F}}\left(\theta_{1}, \theta_{2}, \dot{\theta}_{1}, \dot{\theta}_{2}, .\right) \\
+\mathbf{f}_{\mathscr{F}}\left(\mathbf{\Phi}\left(\theta_{1}, \theta_{2}, .\right)\right)-\mathbf{f}_{\mathscr{F}} \\
\mathbf{g}^{N L}\left(\theta_{1}, \theta_{2}\right)=\mathbf{G}\left(\theta_{1}, \theta_{2}, \dot{\theta}_{1}, \dot{\theta}_{2}\right) \\
\mathbf{s}^{N L}\left(\theta_{1}, \theta_{2}, \mathbf{v}, q\right)=\mathbf{S}\left(\theta_{1}, \theta_{2}, \mathbf{w}+\mathbf{v}, p_{\mathbf{w}}+q\right)-\mathbf{L}_{\mathbf{S}}\left(\theta_{1}, \theta_{2}\right)
\end{array}\right.
$$

where $\mathbf{F}, \mathbf{G}$ and $\mathbf{S}$ are defined as follows

$$
\begin{aligned}
& \mathbf{F}\left(\theta_{1}, \theta_{2}, \mathbf{v}, q\right)=\mathbf{F}^{1}\left(\theta_{1}, \theta_{2}, \mathbf{v}\right)+\mathbf{F}^{2}\left(\theta_{1}, \theta_{2}, \mathbf{v}\right)+\mathbf{F}^{3}\left(\theta_{1}, \theta_{2}, \mathbf{v}\right)+\mathbf{F}^{4}\left(\theta_{1}, \theta_{2}, \mathbf{v}\right)+\mathbf{F}^{5}\left(\theta_{1}, \theta_{2}, q\right), \\
& \mathbf{F}^{1}\left(\theta_{1}, \theta_{2}, \mathbf{v}\right)=\left(\mathrm{I}-\operatorname{cof}\left(\mathcal{J}_{\mathbf{\Psi}}\left(\theta_{1}, \theta_{2}, \boldsymbol{\Phi}\right)\right)^{T}\right) \frac{\partial \mathbf{v}}{\partial t}, \\
& \mathbf{F}^{2}\left(\theta_{1}, \theta_{2}, \mathbf{v}\right)=-\operatorname{cof}\left(\dot{\theta}_{1} \nabla_{\mathbf{x}} \partial_{\theta_{1}} \mathbf{\Psi}\left(\theta_{1}, \theta_{2}, \boldsymbol{\Phi}\right)+\dot{\theta}_{2} \nabla_{\mathbf{x}} \partial_{\theta_{2}} \mathbf{\Psi}\left(\theta_{1}, \theta_{2}, \boldsymbol{\Phi}\right)\right)^{T} \mathbf{v} \\
& -\operatorname{cof}\left(\mathcal{J}_{\mathbf{\Psi}}\left(\theta_{1}, \theta_{2}, \boldsymbol{\Phi}\right)\right)^{T}\left(\nabla_{\mathbf{y}} \mathbf{v}\right)\left(\dot{\theta}_{1} \partial_{\theta_{1}} \mathbf{\Psi}\left(\theta_{1}, \theta_{2}, \boldsymbol{\Phi}\right)+\dot{\theta}_{2} \partial_{\theta_{2}} \mathbf{\Psi}\left(\theta_{1}, \theta_{2}, \boldsymbol{\Phi}\right)\right), \\
& \mathbf{F}^{3}\left(\theta_{1}, \theta_{2}, \mathbf{v}\right)_{i}=\nu \sum_{j, k, \ell, m} \operatorname{cof}\left(\mathcal{J}_{\Psi}\left(\theta_{1}, \theta_{2}, \boldsymbol{\Phi}\right)\right)_{k i} \frac{\partial^{2} v_{k}}{\partial y_{\ell} \partial y_{m}} \frac{\partial \Psi_{\ell}}{\partial x_{j}}\left(\theta_{1}, \theta_{2}, \boldsymbol{\Phi}\right) \frac{\partial \Psi_{m}}{\partial x_{j}}\left(\theta_{1}, \theta_{2}, \boldsymbol{\Phi}\right) \\
& +2 \nu \sum_{j, k, \ell} \operatorname{cof}\left(\partial_{x_{j}} \mathcal{J}_{\boldsymbol{\Psi}}\left(\theta_{1}, \theta_{2}, \boldsymbol{\Phi}\right)\right)_{k i} \frac{\partial v_{k}}{\partial y_{\ell}} \frac{\partial \Psi_{\ell}}{\partial x_{j}}\left(\theta_{1}, \theta_{2}, \boldsymbol{\Phi}\right) \\
& +\nu \sum_{j, k, \ell} \operatorname{cof}\left(\mathcal{J}_{\Psi}\left(\theta_{1}, \theta_{2}, \boldsymbol{\Phi}\right)\right)_{k i} \frac{\partial v_{k}}{\partial y_{\ell}} \frac{\partial^{2} \Psi_{\ell}}{\partial x_{j}^{2}}\left(\theta_{1}, \theta_{2}, \boldsymbol{\Phi}\right) \\
& +\nu \sum_{j, k} \operatorname{cof}\left(\partial_{x_{j}}^{2} \mathcal{J}_{\Psi}\left(\theta_{1}, \theta_{2}, \mathbf{\Phi}\right)\right)_{k i} v_{k}-\nu \Delta_{\mathbf{y}} v_{i}(t, \mathbf{y}) \\
& \mathbf{G}\left(\theta_{1}, \theta_{2}, \omega_{1}, \omega_{2}\right)=\sum_{j=1}^{2} \omega_{j}\left(\operatorname{cof}\left(\mathcal{J}_{\mathbf{\Phi}}\left(\theta_{1}, \theta_{2}, \mathbf{y}\right)\right)^{T} \partial_{\theta_{j}} \mathbf{\Phi}\left(\theta_{1}, \theta_{2}, \mathbf{y}\right)-\partial_{\theta_{j}} \mathbf{\Phi}(0,0, \mathbf{y})\right), \\
& \mathbf{S}\left(\theta_{1}, \theta_{2}, \mathbf{v}, q\right)=-\left(\mathcal{M}_{\theta_{1}, \theta_{2}}-\mathcal{M}_{0,0}\right)\left(\begin{array}{c}
\ddot{\theta}_{1} \\
\ddot{\theta}_{2}
\end{array}\right)+\mathbf{M}_{\mathbf{I}}\left(\theta_{1}, \theta_{2}, \dot{\theta}_{1}, \dot{\theta}_{2}\right) \\
& +\left(\begin{array}{l}
\int_{\partial S_{s}}\left|\mathcal{J}_{\boldsymbol{\Phi}}\left(\theta_{1}, \theta_{2}, \gamma_{y}\right) \mathbf{t}_{s}\right|\left[q \mathrm{I}-\nu\left(\mathcal{G}\left(\theta_{1}, \theta_{2}, \mathbf{v}\right)+\mathcal{G}\left(\theta_{1}, \theta_{2}, \mathbf{v}\right)^{T}\right)\right] \mathbf{n}_{\theta_{1}, \theta_{2}}(\mathbf{\Phi}) \cdot \partial_{\theta_{1}} \mathbf{\Phi}\left(\theta_{1}, \theta_{2}, \gamma_{y}\right) \\
\int_{\partial S_{s}}\left|\mathcal{J}_{\boldsymbol{\Phi}}\left(\theta_{1}, \theta_{2}, \gamma_{y}\right) \mathbf{t}_{s}\right|\left[q \mathrm{I}-\nu\left(\mathcal{G}\left(\theta_{1}, \theta_{2}, \mathbf{v}\right)+\mathcal{G}\left(\theta_{1}, \theta_{2}, \mathbf{v}\right)^{T}\right)\right] \mathbf{n}_{\theta_{1}, \theta_{2}}(\mathbf{\Phi}) \cdot \partial_{\theta_{2}} \mathbf{\Phi}\left(\theta_{1}, \theta_{2}, \gamma_{y}\right)
\end{array}\right)
\end{aligned}
$$

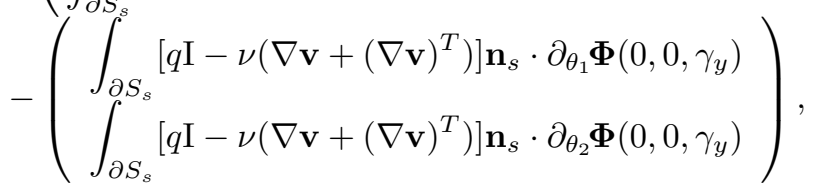

where $\mathbf{M}_{\mathbf{I}}$ and $\mathcal{M}_{\theta_{1}, \theta_{2}}$ are defined in (1.11), (1.12) and

$$
\left.\mathcal{G}\left(\theta_{1}, \theta_{2}, \mathbf{v}\right)_{i j}=\sum_{k} \operatorname{cof}\left[\partial_{x_{j}} \mathcal{J}_{\boldsymbol{\Psi}}\left(\theta_{1}, \theta_{2}, .\right)\right) \circ \boldsymbol{\Phi}\right]_{k i} v_{k}+\sum_{k, \ell} \operatorname{cof}\left(\mathcal{J}_{\boldsymbol{\Psi}}\left(\theta_{1}, \theta_{2}, \boldsymbol{\Phi}\right)\right)_{k i} \frac{\partial v_{k}}{\partial y_{\ell}} \frac{\partial \Psi_{\ell}}{\partial x_{j}}\left(\theta_{1}, \theta_{2}, \boldsymbol{\Phi}\right) .
$$

For the sake of intelligibility, we have used the notation $\mathbf{\Phi}=\mathbf{\Phi}\left(\theta_{1}, \theta_{2},.\right)$. 


\subsection{Proof of the stabilization result in the fixed domain}

In this section, we develop the fixed point argument used to prove the stabilization result of the nonlinear problem (1.15) in the fixed domain $\mathscr{F}_{s}$ (i.e. the stabilization result for $\left.(3.2)-(3.3)\right)$.

Proposition 3.1. Let $\delta>0$ and let $(\mathcal{H})_{\delta}$ be fulfilled. There exists $\varepsilon>0$, such that for every $\left(\mathbf{v}_{0}, \theta_{1,0}, \theta_{2,0}, \omega_{1,0}, \omega_{2,0}\right) \in$ $\mathbf{H}^{1}\left(\mathscr{F}_{s}\right) \times \mathbb{D}_{\Theta} \times \mathbb{R}^{2}$ satisfying the compatibility conditions

$$
\begin{cases}\operatorname{div} \mathbf{v}_{0}=0 & \text { in } \mathscr{F}_{s} \\ \mathbf{v}_{0}=\sum_{j=1}^{2} \omega_{j, 0} \operatorname{cof}\left(\mathcal{J}_{\boldsymbol{\Phi}}\left(\theta_{1,0}, \theta_{2,0}, .\right)\right)^{T} \partial_{\theta_{j}} \mathbf{\Phi}\left(\theta_{1,0}, \theta_{2,0}, .\right) & \text { on } \partial S_{s} \\ \mathbf{v}_{0}=0 & \text { on } \Gamma_{\mathrm{D}}\end{cases}
$$

and

$$
\left\|\mathbf{v}_{0}\right\|_{\mathbf{H}^{1}\left(\mathscr{F}_{s}\right)}+\left|\theta_{1,0}\right|+\left|\theta_{2,0}\right|+\left|\omega_{1,0}\right|+\left|\omega_{2,0}\right| \leq \varepsilon
$$

problem (3.2)-(3.3) admits a solution $\left(\mathbf{v}, q, \theta_{1}, \theta_{2}\right)$ that tends to zero with exponential decay rate $\delta$. For all $t>0$, we have

$$
\|\mathbf{v}(t)\|_{\mathbf{H}^{1}\left(\mathscr{F}_{s}\right)}+\left|\theta_{1}(t)\right|+\left|\theta_{2}(t)\right|+\left|\omega_{1}(t)\right|+\left|\omega_{2}(t)\right| \leq C e^{-\delta t}
$$

Proof. Let $\left(\mathbf{v}_{0}, \theta_{1,0}, \theta_{2,0}, \omega_{1,0}, \omega_{2,0}\right)$ in $\mathbf{H}^{1}\left(\mathscr{F}_{s}\right) \times \mathbb{D}_{\Theta} \times \mathbb{R}^{2}$ fulfilling the compatibility conditions $(3.8)$ and (3.9) for some $\varepsilon>0$. We consider the space

$$
\mathbb{N}_{\delta}^{\infty}=\left\{\left(\mathbf{v}, q, \theta_{1}, \theta_{2}\right) \in \mathbb{U}_{\delta}^{\infty} \times \mathbb{P}_{\delta}^{\infty} \times \Theta_{\delta}^{\infty} \text { with } \begin{array}{l}
\left(\theta_{1}, \theta_{2}, \dot{\theta}_{1}, \dot{\theta}_{2}\right)(0)=\left(\theta_{1,0}, \theta_{2,0}, \omega_{1,0}, \omega_{2,0}\right), \\
\text { for every } t \text { in }(0, \infty),\left(\theta_{1}(t), \theta_{2}(t)\right) \in \mathbb{D}_{\Theta}
\end{array}\right\},
$$

equipped with the natural norm of $\mathbb{U}_{\delta}^{\infty} \times \mathbb{P}_{\delta}^{\infty} \times \Theta_{\delta}^{\infty}$

$$
\left\|\left(\mathbf{v}, q, \theta_{1}, \theta_{2}\right)\right\|_{\mathbb{U}_{\delta}^{\infty} \times \mathbb{P}_{\delta}^{\infty} \times \Theta_{\delta}^{\infty}}=\|\mathbf{v}\|_{\mathbb{U}_{\delta}^{\infty}}+\|q\|_{\mathbb{P}_{\delta}^{\infty}}+\left\|\left(\theta_{1}, \theta_{2}\right)\right\|_{\Theta_{\delta}^{\infty}}
$$

where all the spaces used are defined in $(2.4)-(2.9)$.

We define the application $\Lambda^{\infty}$ on $\mathbb{N}_{\delta}^{\infty}$ as follows. For $\left(\overline{\mathbf{v}}, \bar{q}, \bar{\theta}_{1}, \bar{\theta}_{2}\right) \in \mathbb{N}_{\delta}^{\infty},\left(\mathbf{v}, q, \theta_{1}, \theta_{2}\right)=\Lambda^{\infty}\left(\overline{\mathbf{v}}, \bar{q}_{1}, \bar{\theta}_{1}, \bar{\theta}_{2}\right) \in$ $\mathbb{U}_{\delta}^{\infty} \times \mathbb{P}_{\delta}^{\infty} \times \Theta_{\delta}^{\infty}$ is the solution to problem (3.2) where the source terms are $\mathbf{f}=\mathbf{f}^{N L}\left(\bar{\theta}_{1}, \bar{\theta}_{2}, \overline{\mathbf{v}}_{\bar{q}}\right), \mathbf{g}=\mathbf{g}^{N L}\left(\bar{\theta}_{1}, \bar{\theta}_{2}\right)$ and $\mathbf{s}=\mathbf{s}^{N L}\left(\bar{\theta}_{1}, \bar{\theta}_{2}, \overline{\mathbf{v}}, \bar{q}\right)$ (defined in (3.4)). Note that $\Lambda^{\infty}$ depends on the initial data.

The compatibility conditions (3.8) correspond to (2.10) with $\mathbf{g}(0)=\mathbf{G}\left(\theta_{1,0}, \theta_{2,0}, \omega_{1,0}, \omega_{2,0}\right)$ and the conditions in (3.11) impose that $\mathbf{g}^{N L}\left(\bar{\theta}_{1}, \bar{\theta}_{2}, \overline{\mathbf{v}}, \bar{q}\right)(0)=\mathbf{G}\left(\theta_{1,0}, \theta_{2,0}, \omega_{1,0}, \omega_{2,0}\right)$. Moreover, we prove in (3.12) that, for every $\left(\bar{\theta}_{1}, \bar{\theta}_{2}, \overline{\mathbf{v}}, \bar{q}\right) \in \mathbb{N}_{\delta}^{\infty}$, we have $\mathbf{f}^{N L}\left(\bar{\theta}_{1}, \bar{\theta}_{2}, \overline{\mathbf{v}}, \bar{q}\right) \in \mathbb{F}_{\delta}^{\infty}, \mathbf{g}^{N L}\left(\bar{\theta}_{1}, \bar{\theta}_{2}\right) \in \mathbb{G}_{\delta}^{\infty}$ and $\mathbf{s}^{N L}\left(\bar{\theta}_{1}, \bar{\theta}_{2}, \overline{\mathbf{v}}, \bar{q}\right) \in \mathbb{S}_{\delta}^{\infty}$. Hence, according to Proposition 2.1, $\Lambda^{\infty}$ is well defined.

The domain $\mathbb{D}_{\Theta}$ is open and $(0,0) \in \mathbb{D}_{\Theta}$, then there exists $R_{0}>0$ such that $B\left((0,0), R_{0}\right) \subset \mathbb{D}_{\Theta}$. The application $\Lambda^{\infty}$ will be studied on a ball of radius $R \leq R_{0}$

$$
\mathbb{B}_{R}^{\infty}=\left\{\left(\mathbf{v}, q, \theta_{1}, \theta_{2}\right) \in \mathbb{N}_{\delta}^{\infty} \text { with }\left\|\left(\mathbf{v}, q, \theta_{1}, \theta_{2}\right)\right\|_{\mathbb{U}_{\delta}^{\infty} \times \mathbb{P}_{\delta}^{\infty} \times \Theta_{\delta}^{\infty}} \leq R\right\}
$$

We also consider the space

$\widetilde{\mathbb{B}_{R}^{\infty}}=\left\{\left(\mathbf{v}, q, \theta_{1}, \theta_{2}\right) \in \mathbb{U}_{\delta}^{\infty} \times \mathbb{P}_{\delta}^{\infty} \times \Theta_{\delta}^{\infty}\right.$ with $\left.\left\|\left(\mathbf{v}, q, \theta_{1}, \theta_{2}\right)\right\|_{\mathbb{U}_{\delta}^{\infty} \times \mathbb{P}_{\delta}^{\infty} \times \Theta_{\delta}^{\infty}} \leq R, \quad \forall t \in(0, \infty),\left(\theta_{1}, \theta_{2}\right)(t) \in \mathbb{D}_{\Theta}\right\}$ in which the initial value for $\left(\theta_{1}, \theta_{2}, \dot{\theta}_{1}, \dot{\theta}_{2}\right)$ is free. The following lemma will be used.

Lemma 3.2. There exists a constant $C^{\prime}=C^{\prime}\left(R_{0}\right)$, such that for every $R \in\left(0, R_{0}\right)$, every $\left(\mathbf{v}^{a}, q^{a}, \theta_{1}^{a}, \theta_{2}^{a}\right)$ and $\left(\mathbf{v}^{b}, q^{b}, \theta_{1}^{b}, \theta_{2}^{b}\right) \in \widetilde{\mathbb{B}_{R}^{\infty}}$, the following estimates hold

$$
\begin{aligned}
& \left\|\mathbf{f}^{N L}\left(\theta_{1}^{a}, \theta_{2}^{a}, \mathbf{v}^{a}, q^{a}\right)-\mathbf{f}^{N L}\left(\theta_{1}^{b}, \theta_{2}^{b}, \mathbf{v}^{b}, q^{b}\right)\right\|_{\mathbb{F}_{\delta}^{\infty}} \leq C^{\prime} R\left(\left\|\mathbf{v}^{a}-\mathbf{v}^{b}\right\|_{\mathbb{U}_{\delta}^{\infty}}+\left\|q^{a}-q^{b}\right\|_{\mathbb{P}_{\delta}^{\infty}}+\left\|\theta^{a}-\theta^{b}\right\|_{\Theta_{\delta}^{\infty}}\right), \\
& \left\|\mathbf{g}^{N L}\left(\theta_{1}^{a}, \theta_{2}^{a}\right)-\mathbf{g}^{N L}\left(\theta_{1}^{b}, \theta_{2}^{b}\right)\right\|_{\mathbb{G}_{\delta}^{\infty}} \leq C^{\prime} R\left\|\theta^{a}-\theta^{b}\right\|_{\Theta_{\delta}^{\infty}}, \\
& \left\|\mathbf{s}^{N L}\left(\theta_{1}^{a}, \theta_{2}^{a}, \mathbf{v}^{a}, q^{a}\right)-\mathbf{s}^{N L}\left(\theta_{1}^{b}, \theta_{2}^{b}, \mathbf{v}^{b}, q^{b}\right)\right\|_{\mathbb{S}_{\delta}^{\infty}} \leq C^{\prime} R\left(\left\|\mathbf{v}^{a}-\mathbf{v}^{b}\right\|_{\mathbb{U}_{\delta}^{\infty}}+\left\|q^{a}-q^{b}\right\|_{\mathbb{P}_{\delta}^{\infty}}+\left\|\theta^{a}-\theta^{b}\right\|_{\Theta_{\delta}^{\infty}}\right) .
\end{aligned}
$$

This lemma is proven in Appendix C.2. We use the spaces $\mathbf{H}_{\beta}^{2}\left(\mathscr{F}_{s}\right)$ and $\mathrm{H}_{\beta}^{1}\left(\mathscr{F}_{s}\right)$ in this proof.

We now denote $C$ the constant in (2.11) and $C^{\prime}$ the one in Lemma 3.2.

At this point, we use Lemma 3.2 with $\left(\mathbf{v}^{b}, q^{b}, \theta_{1}^{b}, \theta_{2}^{b}\right)=(0,0,0,0)$, we thus get for every $R>0$ and for every $\left(\mathbf{v}, q, \theta_{1}, \theta_{2}\right)$ in $\mathbb{B}_{R}^{\infty}$,

$$
\left\|\mathbf{f}^{N L}\left(\theta_{1}, \theta_{2}, \mathbf{v}, q\right)\right\|_{\mathbb{F}_{\delta}^{\infty}}+\left\|\mathbf{g}^{N L}\left(\theta_{1}, \theta_{2}\right)\right\|_{\mathbb{G}_{\delta}^{\infty}}+\left\|\mathbf{s}^{N L}\left(\theta_{1}, \theta_{2}, \mathbf{v}, q\right)\right\|_{\mathbb{S}_{\delta}^{\infty}} \leq 3 C^{\prime} R^{2} .
$$


Let $R=\min \left(R_{0}, 1 /\left(6 C C^{\prime}\right)\right)$ and $\varepsilon=R /(2 C)$, hence $3 C C^{\prime} R^{2} \leq R / 2$.

We can build $\left(\theta_{1}, \theta_{2}\right)$ as the solution to

$$
\left\{\begin{array}{l}
\ddot{\theta}_{j}(t)+2 \delta \dot{\theta}_{j}(t)+2 \delta^{2} \theta_{j}(t)=0, \quad t>0, \\
\theta_{j}(0)=\theta_{j, 0}, \quad \dot{\theta}_{j}(0)=\omega_{j, 0} .
\end{array}\right.
$$

For $\varepsilon$ small enough and $\left|\omega_{j, 0}\right|+\left|\theta_{j, 0}\right| \leq \varepsilon,\left(0,0, \theta_{1}, \theta_{2}\right)$ belongs to $\mathbb{B}_{R}^{\infty}$. Thus, taking $\varepsilon>0$ smaller if necessary, we can assume that $\mathbb{B}_{R}^{\infty} \neq \emptyset$. In the sequel, we choose such a $\varepsilon$.

According to Proposition 2.1, we have for every $\left(\mathbf{v}, q, \theta_{1}, \theta_{2}\right)$ in $\mathbb{B}_{R}^{\infty}$,

$$
\begin{array}{r}
\left.\left\|\Lambda^{\infty}\left(\mathbf{v}, q, \theta_{1}, \theta_{2}\right)\right\|_{\mathbb{U}_{\delta}^{\infty} \times \mathbb{P}_{\delta}^{\infty} \times \Theta_{\delta}^{\infty} \leq C\left(\left\|\mathbf{v}_{0}\right\|_{\mathbf{H}^{1}\left(\mathscr{F}_{s}\right)}+\left|\theta_{1,0}\right|+\left|\theta_{2,0}\right|+\left|\omega_{1,0}\right|+\left|\omega_{2,0}\right|+\left\|\mathbf{f}^{N L}\left(\theta_{1}, \theta_{2}, \mathbf{v}, q\right)\right\|_{\mathbb{F}_{\delta}^{\infty}}\right.}+\left\|\mathbf{g}^{N L}\left(\theta_{1}, \theta_{2}\right)\right\|_{\mathbb{G}_{\delta}^{\infty}}+\left\|\mathbf{s}^{N L}\left(\theta_{1}, \theta_{2}, \mathbf{v}, q\right)\right\|_{\mathbb{S}_{\delta}^{\infty}}\right)
\end{array}
$$

we combine this result with (3.12). Then, by using $(3.9), \varepsilon=R /(2 C)$ and $3 C C^{\prime} R^{2} \leq R / 2$, we have

$$
\left\|\Lambda^{\infty}\left(\mathbf{v}, q, \theta_{1}, \theta_{2}\right)\right\|_{\mathbb{U}_{\delta}^{\infty} \times \mathbb{P}_{\delta}^{\infty} \times \Theta_{\delta}^{\infty}} \leq R
$$

Hence, if we write $\left(\tilde{\mathbf{v}}, \tilde{q}, \tilde{\theta}_{1}, \tilde{\theta}_{2}\right)=\Lambda^{\infty}\left(\theta_{1}, \theta_{2}, \mathbf{v}, q\right)$, then we have $\left\|\left(\tilde{\theta}_{1}, \tilde{\theta}_{2}\right)\right\|_{\mathbf{L}^{\infty}(0, \infty)} \leq\left\|\Lambda^{\infty}\left(\theta_{1}, \theta_{2}, \mathbf{v}, q\right)\right\|_{\mathbb{N}_{\delta}^{\infty}} \leq$ $R \leq R_{0}$. Then, $\left(\tilde{\theta}_{1}, \tilde{\theta}_{2}\right)$ belongs to $\mathbb{D}_{\Theta}$. This proves that $\Lambda^{\infty}: \mathbb{B}_{R}^{\infty} \rightarrow \mathbb{B}_{R}^{\infty}$.

For $\left(\mathbf{v}^{a}, q^{a}, \theta_{1}^{a}, \theta_{2}^{a}\right)$ and $\left(\mathbf{v}^{b}, q^{b}, \theta_{1}^{b}, \theta_{2}^{b}\right)$ in $\mathbb{B}_{R}^{\infty}, \Lambda^{\infty}\left(\mathbf{v}^{a}, q^{a}, \theta_{1}^{a}, \theta_{2}^{a}\right)-\Lambda^{\infty}\left(\mathbf{v}^{b}, q^{b}, \theta_{1}^{b}, \theta_{2}^{b}\right)$ solves problem (3.2) where the source terms are $\mathbf{f}^{N L}\left(\theta_{1}^{a}, \theta_{2}^{a}, \mathbf{v}^{a}, q^{a}\right)-\mathbf{f}^{N L}\left(\theta_{1}^{b}, \theta_{2}^{b}, \mathbf{v}^{b}, q^{b}\right), \mathbf{g}^{N L}\left(\theta_{1}^{a}, \theta_{2}^{a}\right)-\mathbf{g}^{N L}\left(\theta_{1}^{b}, \theta_{2}^{b}\right), \mathbf{s}^{N L}\left(\theta_{1}^{a}, \theta_{2}^{a}, \mathbf{v}^{a}, q^{a}\right)-$ $\mathbf{s}^{N L}\left(\theta_{1}^{b}, \theta_{2}^{b}, \mathbf{v}^{b}, q^{b}\right)$ and the initial data are null. Then, according to Proposition 2.1 and Lemma 3.2, we have

$$
\left\|\Lambda^{\infty}\left(\mathbf{v}^{a}, q^{a}, \theta_{1}^{a}, \theta_{2}^{a}\right)-\Lambda^{\infty}\left(\mathbf{v}^{b}, q^{b}, \theta_{1}^{b}, \theta_{2}^{b}\right)\right\|_{\mathbb{U}_{\delta}^{\infty} \times \mathbb{P}_{\delta}^{\infty} \times \Theta_{\delta}^{\infty}} \leq 3 C C^{\prime} R\left(\left\|\theta^{a}-\theta^{b}\right\|_{\Theta_{\delta}^{\infty}}+\left\|\mathbf{v}^{a}-\mathbf{v}^{b}\right\|_{\mathbb{U}_{\delta}^{\infty}}+\left\|q^{a}-q^{b}\right\|_{\mathbb{P}_{\delta}^{\infty}}\right) .
$$

As $R \leq 1 /\left(6 C C^{\prime}\right)$, the estimate (3.13) yields that $\Lambda^{\infty}$ is a contraction on $\mathbb{B}_{R}^{\infty}$. Hence, according to the Picard fixed point theorem, there exists a unique fixed point $\left(\mathbf{v}, q, \theta_{1}, \theta_{2}\right)$ to $\Lambda^{\infty}$ in $\mathbb{B}_{R}^{\infty}$. This fixed point solves the closed-loop nonlinear problem (3.2)-(3.3).

Estimate (3.10) is then a consequence of the fact that $\left(\mathbf{v}, q, \theta_{1}, \theta_{2}\right) \in \mathbb{B}_{R}^{\infty}$ :

$$
\left\|\mathbf{v} e^{\delta t}\right\|_{\mathscr{C}^{0}\left([0, \infty) ; \mathbf{H}^{1}\left(\mathscr{F}_{s}\right)\right)}+\left\|\theta e^{\delta t}\right\|_{\mathrm{L}^{\infty}\left(0, \infty ; \mathbb{D}_{\Theta}\right)}+\left\|\dot{\theta} e^{\delta t}\right\|_{L^{\infty}\left(0, \infty ; \mathbb{R}^{2}\right)} \leq R \leq R_{0}
$$

\subsection{Proof of Theorem 1.6}

Proof. The last step towards proving Theorem 1.6 is to use the change of variables (3.1) to prove the result on $(\mathbf{u}, p)$ as a consequence of Proposition 3.1 that states properties of $(\mathbf{v}, q)$. The only difficulty is to handle the nonlinear term $\operatorname{cof}\left(\mathcal{J}_{\boldsymbol{\Phi}}\right)^{T}$ that is present in this change of variables. This nonlinearity creates some difficulties for using the smallness assumption and compatibility conditions on the initial data.

Let $\delta>0$, we consider that $(\mathcal{H})_{\delta}$ is fulfilled and that the initial data satisfy the compatibility conditions (1.24). Moreover, we consider that for some $\varepsilon_{1}>0$ we have

$$
\left\|\mathbf{u}_{0}\left(\boldsymbol{\Phi}\left(\theta_{1,0}, \theta_{2,0}, .\right)\right)-\mathbf{w}(.)\right\|_{\mathbf{H}^{1}\left(\mathscr{F}_{s}\right)}+\left|\theta_{1,0}\right|+\left|\theta_{2,0}\right|+\left|\omega_{1,0}\right|+\left|\omega_{2,0}\right| \leq \varepsilon_{1} .
$$

Since $\mathbf{v}_{0}(\mathbf{y})=\operatorname{cof}\left(\mathcal{J}_{\mathbf{\Phi}}\left(\theta_{1,0}, \theta_{2,0}, \mathbf{y}\right)\right)^{T} \mathbf{u}_{0} \circ \mathbf{\Phi}\left(\theta_{1,0}, \theta_{2,0}, \mathbf{y}\right)-\mathbf{w}(\mathbf{y})$, we have $(3.8)$ and

$$
\begin{aligned}
\left\|\mathbf{v}_{0}\right\|_{\mathbf{H}^{1}\left(\mathscr{F}_{s}\right)} & \leq\left\|\operatorname{cof}\left(\mathcal{J}_{\boldsymbol{\Psi}}\left(\theta_{1,0}, \theta_{2,0}, \boldsymbol{\Phi}\right)\right)^{T}\left(\mathbf{u}_{0} \circ \mathbf{\Phi}-\mathbf{w}\right)\right\|_{\mathbf{H}^{1}\left(\mathscr{F}_{s}\right)}+\| \operatorname{cof}\left(\mathcal{J}_{\mathbf{\Psi}}\left(\theta_{1,0}, \theta_{2,0}, \boldsymbol{\Phi}\right)-\mathrm{I}\right)^{T} \mathbf{w}_{\mathbf{H}^{1}\left(\mathscr{F}_{s}\right)} \\
& \leq K \varepsilon_{1}+K\|\mathbf{w}\|_{\mathbf{H}_{\beta}^{2}\left(\mathscr{F}_{s}\right)} \varepsilon_{1},
\end{aligned}
$$

where $K$ is the constant in (C.18). In order to fulfil (3.9), we choose

$$
\varepsilon_{1}=\frac{\varepsilon}{K+K\|\mathbf{w}\|_{\mathbf{H}_{\beta}^{2}\left(\mathscr{F}_{s}\right)}},
$$

where $\varepsilon>0$ is the bound in Proposition 3.1, we have (3.9). Then, according to Proposition 3.1, the feedback $\mathbf{h}=\mathcal{K}_{\delta}\left(\mathbf{v}, \theta_{1}, \theta_{2}, \dot{\theta}_{1}, \dot{\theta}_{2}\right)$ stabilizes problem $(3.2)-(3.3)$ at decay rate $\delta$. We denote $\left(\mathbf{v}, q, \theta_{1}, \theta_{2}\right)$ the solution to $(3.2)-(3.3)$.

We use the identity

$$
\begin{aligned}
& \forall t \in(0, \infty), \forall \mathbf{x} \in \mathscr{F}\left(\theta_{1}(t), \theta_{2}(t)\right) \\
& \qquad\left\{\begin{array}{l}
\mathbf{u}(t, \mathbf{x})=\operatorname{cof}\left(\mathcal{J}_{\mathbf{\Psi}}\left(\theta_{1}(t), \theta_{2}(t), \mathbf{x}\right)\right)^{T}\left(\mathbf{v}\left(t, \Psi\left(\theta_{1}(t), \theta_{2}(t), \mathbf{x}\right)\right)+\mathbf{w}\left(\mathbf{\Psi}\left(\theta_{1}(t), \theta_{2}(t), \mathbf{x}\right)\right)\right) \\
p(t, \mathbf{x})=q\left(t, \mathbf{\Psi}\left(\theta_{1}(t), \theta_{2}(t), \mathbf{x}\right)\right)+p_{\mathbf{w}} \circ \mathbf{\Psi}\left(\theta_{1}(t), \theta_{2}(t), \mathbf{x}\right)
\end{array}\right.
\end{aligned}
$$


The quadruplet $\left(\mathbf{u}, p, \theta_{1}, \theta_{2}\right)$ is solution to (1.15) where

$$
\mathbf{h}=\mathcal{K}_{\delta}\left(\operatorname{cof}\left(\mathcal{J}_{\boldsymbol{\Phi}}\left(\theta_{1}(t), \theta_{2}(t), .\right)\right)^{T} \mathbf{u}\left(t, \boldsymbol{\Phi}\left(\theta_{1}(t), \theta_{2}(t), .\right)\right)-\mathbf{w}(.), \theta_{1}(t), \theta_{2}(t), \dot{\theta}_{1}(t), \dot{\theta}_{2}(t)\right) .
$$

Moreover, for $\varepsilon$ small enough and for every $t>0$, we have the estimate

$$
\begin{aligned}
\left\|\mathbf{u}\left(t, \boldsymbol{\Phi}\left(\theta_{1}(t), \theta_{2}(t), .\right)\right)-\mathbf{w}\right\|_{\mathbf{H}^{1}\left(\mathscr{F}_{s}\right)} & \leq\left\|\operatorname{cof}\left(\mathcal{J}_{\mathbf{\Psi}}\left(\theta_{1}(t), \theta_{2}(t), .\right)\right)-\mathrm{I}\right\|_{\mathbf{H}^{2}\left(\mathscr{F}_{s}\right)}\|\mathbf{v}(t)+\mathbf{w}\|_{\mathbf{H}^{1}\left(\mathscr{F}_{s}\right)}+\|\mathbf{v}(t)\|_{\mathbf{H}^{1}\left(\mathscr{F}_{s}\right)} \\
& \leq K\left(\left|\left(\theta_{1}(t), \theta_{2}(t)\right)\right|+\|\mathbf{v}(t)\|_{\mathbf{H}^{1}\left(\mathscr{F}_{s}\right)}\right) .
\end{aligned}
$$

Hence the estimate (3.10) implies that

$$
\forall t>0, \quad\left\|\mathbf{u}\left(t, \boldsymbol{\Phi}\left(\theta_{1}(t), \theta_{2}(t), .\right)\right)-\mathbf{w}(.)\right\|_{\mathbf{H}^{1}\left(\mathscr{F}_{s}\right)}+\left|\theta_{1}(t)\right|+\left|\theta_{2}(t)\right|+\left|\dot{\theta}_{1}(t)\right|+\left|\dot{\theta}_{2}(t)\right| \leq C e^{-\delta t} .
$$

This concludes the proof of Theorem 1.6.

\section{Appendix}

\section{A The linearized terms}

In what follows we give the explicit expression of the functions $\mathbf{L}_{1}-\mathbf{L}_{4}$ and of the constants $\mathbf{L}_{5}-\mathbf{L}_{6}$. We denote $\mathbf{t}_{s}=\mathbf{n}_{s}^{\perp}=\left(-\left(\mathbf{n}_{s}\right)_{2},\left(\mathbf{n}_{s}\right)_{1}\right)$ a unit tangent vector to $\partial S_{s}$. We have

$$
\begin{aligned}
& \mathbf{L}_{1}(\mathbf{y})_{i}=\left(\nu \mathbf{L}_{\mathbf{F}^{3}}(\mathbf{y})+\mathbf{L}_{\mathbf{F}^{4}}(\mathbf{y})+\mathbf{L}_{\mathbf{F}^{5}}(\mathbf{y})\right)_{i 1}+\left(\nabla \mathbf{f}_{\mathscr{F}}(\mathbf{y}) \partial_{\theta_{1}} \boldsymbol{\Phi}(0,0, \mathbf{y})\right)_{i}, \\
& \mathbf{L}_{2}(\mathbf{y})_{i}=\left(\nu \mathbf{L}_{\mathbf{F}^{3}}(\mathbf{y})+\mathbf{L}_{\mathbf{F}^{4}}(\mathbf{y})+\mathbf{L}_{\mathbf{F}^{5}}(\mathbf{y})\right)_{i 2}+\left(\nabla \mathbf{f}_{\mathscr{F}}(\mathbf{y}) \partial_{\theta_{2}} \boldsymbol{\Phi}(0,0, \mathbf{y})\right)_{i}, \\
& \mathbf{L}_{3}(\mathbf{y})_{i}=\left(\mathbf{L}_{\mathbf{F}^{2}}(\mathbf{y})\right)_{i 1}, \\
& \mathbf{L}_{4}(\mathbf{y})_{i}=\left(\mathbf{L}_{\mathbf{F}^{2}}(\mathbf{y})\right)_{i 2}, \\
& \left(\mathbf{L}_{5}\right)_{i}=\int_{\partial S_{s}}\left(-\sigma_{F}\left(\mathbf{w}, p_{\mathbf{w}}\right) \mathbf{n}_{s}\right) \cdot \partial_{\theta_{1} \theta_{i}} \mathbf{\Phi}\left(0,0, \gamma_{y}\right)-\sum_{k, \ell} \sigma_{F}\left(\mathbf{w}, p_{\mathbf{w}}\right)_{\ell k}\left(\mathbf{L}_{\mathbf{n}_{\theta_{1}, \theta_{2}}}\right)_{k 1} \partial_{\theta_{i}} \Phi_{\ell}\left(0,0, \gamma_{y}\right) \\
& -\left(\nabla_{\mathbf{y}} \partial_{\theta_{1}} \mathbf{\Phi}\left(0,0, \gamma_{y}\right) \mathbf{t}_{s} \cdot \mathbf{t}_{s}\right) \sigma_{F}\left(\mathbf{w}, p_{\mathbf{w}}\right) \mathbf{n}_{s} \cdot \partial_{\theta_{i}} \mathbf{\Phi}\left(0,0, \gamma_{y}\right) \\
& -\nu \sum_{k, \ell}\left(\left(\mathbf{L}_{\mathcal{G}}\right)_{k \ell 1}+\left(\mathbf{L}_{\mathcal{G}}\right)_{\ell k 1}\right)\left(\mathbf{n}_{s}\right)_{k} \partial_{\theta_{i}} \Phi_{\ell}\left(0,0, \gamma_{y}\right) \mathrm{d} \gamma_{y} \text {, } \\
& \left(\mathbf{L}_{6}\right)_{i}=\int_{\partial S_{s}}\left(-\sigma_{F}\left(\mathbf{w}, p_{\mathbf{w}}\right) \mathbf{n}_{s}\right) \cdot \partial_{\theta_{2} \theta_{i}} \mathbf{\Phi}\left(0,0, \gamma_{y}\right)-\sum_{k, \ell} \sigma_{F}\left(\mathbf{w}, p_{\mathbf{w}}\right)_{\ell k}\left(\mathbf{L}_{\mathbf{n}_{\theta_{1}, \theta_{2}}}\right)_{k 2} \partial_{\theta_{i}} \Phi_{\ell}\left(0,0, \gamma_{y}\right) \\
& -\left(\nabla_{\mathbf{y}} \partial_{\theta_{2}} \mathbf{\Phi}\left(0,0, \gamma_{y}\right) \mathbf{t}_{s} \cdot \mathbf{t}_{s}\right) \sigma_{F}\left(\mathbf{w}, p_{\mathbf{w}}\right) \mathbf{n}_{s} \cdot \partial_{\theta_{i}} \boldsymbol{\Phi}\left(0,0, \gamma_{y}\right) \\
& -\nu \sum_{k, \ell}\left(\left(\mathbf{L}_{\mathcal{G}}\right)_{k \ell 2}+\left(\mathbf{L}_{\mathcal{G}}\right)_{\ell k 2}\right)\left(\mathbf{n}_{s}\right)_{k} \partial_{\theta_{i}} \Phi_{\ell}\left(0,0, \gamma_{y}\right) \mathrm{d} \gamma_{y}
\end{aligned}
$$

where the terms used are defined in (A.7)-(A.12). In the previous paragraph, we denoted by $\left(\mathbf{L}_{\mathbf{F}^{k}}\right)_{i j}$ for $3 \leq k \leq 5$, the linearization of $\left(\mathbf{F}^{k}\right)_{i}$ (defined in (3.6)) around the stationary state $\left(\mathbf{w}, p_{\mathbf{w}}, 0,0\right)$ solution of $(1.17)$ with respect to the parameter $\theta_{j}$ and $\left(\mathbf{L}_{\mathbf{F}^{2}}\right)_{i j}$ is the linearization of $\left(\mathbf{F}^{2}\right)_{i}$ with respect to $\dot{\theta}_{j}$. These terms are given below

$$
\begin{aligned}
\left(\mathbf{L}_{\mathbf{F}^{2}}(\mathbf{y})\right)_{i j}=\left(\left(\partial_{\theta_{j}} \mathbf{\Phi}(0,0, \mathbf{y}) \cdot \nabla_{\mathbf{y}}\right) \mathbf{w}+\operatorname{cof}\left(\nabla_{\mathbf{y}} \partial_{\theta_{j}} \mathbf{\Phi}(0,0, \mathbf{y})\right)^{T} \mathbf{w}\right)_{i} & \\
\left(\mathbf{L}_{\mathbf{F}^{3}}(\mathbf{y})\right)_{i j}= & -2 \nabla_{\mathbf{y}} \partial_{\theta_{j}} \mathbf{\Phi}(0,0, \mathbf{y}): \nabla^{2} w_{i}(\mathbf{y})-\left(\operatorname{cof}\left(\nabla_{\mathbf{y}} \partial_{\theta_{j}} \mathbf{\Phi}(0,0, \mathbf{y})\right)^{T} \Delta \mathbf{w}(\mathbf{y})\right)_{i} \\
& +2 \sum_{k, \ell} \operatorname{cof}\left(\frac{\partial}{\partial x_{\ell}}\left(\nabla_{\mathbf{x}} \partial_{\theta_{j}} \mathbf{\Psi}(0,0, \mathbf{y})\right)\right)_{k i} \frac{\partial w_{k}}{\partial y_{\ell}}(\mathbf{y}) \\
& +\sum_{\ell, m} \frac{\partial w_{i}}{\partial y_{\ell}}(\mathbf{y}) \frac{\partial^{2}}{\partial x_{m}^{2}}\left(\partial_{\theta_{j}} \mathbf{\Psi}(0,0, \mathbf{y})\right)_{\ell} \\
& +\sum_{k, m} \operatorname{cof}\left(\frac{\partial^{2}}{\partial x_{m}^{2}}\left(\nabla_{\mathbf{x}} \partial_{\theta_{j}} \mathbf{\Psi}(0,0, \mathbf{y})\right)\right)_{k i} w_{k}(\mathbf{y}), \\
\left(\mathbf{L}_{\mathbf{F}^{4}}(\mathbf{y})\right)_{i j}=- & \sum_{k, \ell} \operatorname{cof}\left(\frac{\partial}{\partial x_{k}}\left(\nabla_{\mathbf{x}} \partial_{\theta_{j}} \mathbf{\Psi}(0,0, \mathbf{y})\right)\right)_{\ell i} w_{k}(\mathbf{y}) w_{\ell}(\mathbf{y}) \\
+ & \sum_{k, \ell}\left(2 \operatorname{Tr}\left(\nabla_{\mathbf{y}} \partial_{\theta_{j}} \mathbf{\Phi}(0,0, \mathbf{y})\right) \delta_{i \ell}-\left(\nabla_{\mathbf{y}} \partial_{\theta_{j}} \mathbf{\Phi}(0,0, \mathbf{y})\right)_{i \ell}\right) w_{k}(\mathbf{y}) \frac{\partial w_{\ell}}{\partial y_{k}}(\mathbf{y})
\end{aligned}
$$

and

$$
\left(\mathbf{L}_{\mathbf{F}^{5}}(\mathbf{y})\right)_{i j}=\left(\left(\nabla_{\mathbf{y}} \partial_{\theta_{j}} \mathbf{\Phi}(0,0, \mathbf{y})\right)^{T} \nabla_{\mathbf{y}} p_{\mathbf{w}}(\mathbf{y})\right)_{i}
$$


We also define the linearization of $\mathbf{n}_{\theta_{1}, \theta_{2}}$ (the unit outward normal to $\left.\mathscr{F}\left(\theta_{1}, \theta_{2}\right)\right)$ and $\mathcal{G}($ defined in $(3.7))$ with respect to $\theta_{j}$ by

$$
\left(\mathbf{L}_{\mathbf{n}_{\theta_{1}, \theta_{2}}}\left(\gamma_{y}\right)\right)_{j, n}=\left(\operatorname{cof}\left(\nabla_{\mathbf{y}} \partial_{\theta_{n}} \boldsymbol{\Phi}\left(0,0, \gamma_{y}\right)\right) \mathbf{n}_{s}\right)_{j}-\left(\operatorname{cof}\left(\nabla_{\mathbf{y}} \partial_{\theta_{n}} \boldsymbol{\Phi}\left(0,0, \gamma_{y}\right)\right) \mathbf{n}_{s} \cdot \mathbf{n}_{s}\right)\left(\mathbf{n}_{s}\right)_{j}
$$

and

$$
\begin{aligned}
\left(\mathbf{L}_{\mathcal{G}}\left(\gamma_{y}\right)\right)_{i, j, n}= & \sum_{k} w_{k}\left(\gamma_{y}\right) \operatorname{cof}\left(\frac{\partial}{\partial x_{j}}\left(\nabla_{\mathbf{x}} \partial_{\theta_{n}} \mathbf{\Psi}\left(0,0, \gamma_{y}\right)\right)\right)_{k i} \\
& -\sum_{k} \operatorname{cof}\left(\nabla_{\mathbf{y}} \partial_{\theta_{n}} \mathbf{\Phi}\left(0,0, \gamma_{y}\right)\right)_{k i} \frac{\partial w_{k}}{\partial y_{j}}\left(\gamma_{y}\right)+\sum_{k} \nabla_{\mathbf{y}} \partial_{\theta_{n}} \mathbf{\Phi}\left(0,0, \gamma_{y}\right)_{k j} \frac{\partial w_{i}}{\partial y_{k}}\left(\gamma_{y}\right) .
\end{aligned}
$$

\section{B Independence of the hypothesis $(\mathcal{H})_{\delta}$ with respect to the diffeo- morphism $\Phi$}

Let $\boldsymbol{\Phi}^{a}\left(\theta_{1}, \theta_{2}, \mathbf{y}\right)$ and $\boldsymbol{\Phi}^{b}\left(\theta_{1}, \theta_{2}, \mathbf{y}\right)$ be two diffeomorphisms from $\mathscr{F}_{s}$ to $\mathscr{F}\left(\theta_{1}, \theta_{2}\right)$ that are extensions of $\mathbf{X}\left(\theta_{1}, \theta_{2}, \mathbf{y}\right)$ into the fluid domain. We also assume that $\boldsymbol{\Phi}^{a}(0,0, \mathbf{y})=\boldsymbol{\Phi}^{b}(0,0, \mathbf{y})=\mathbf{y}, \forall \mathbf{y} \in \mathscr{F}_{s}$. Let us denote $\boldsymbol{\Psi}^{a}\left(\theta_{1}, \theta_{2},.\right)$ and $\boldsymbol{\Psi}^{b}\left(\theta_{1}, \theta_{2},.\right)$ the inverse diffeomorphisms of, respectively, $\boldsymbol{\Phi}^{a}\left(\theta_{1}, \theta_{2},.\right)$ and $\boldsymbol{\Phi}^{b}\left(\theta_{1}, \theta_{2},.\right)$. We also denote $\mathcal{J}_{\mathbf{\Phi}}^{a}\left(\theta_{1}, \theta_{2}, \mathbf{y}\right), \mathcal{J}_{\mathbf{\Phi}}^{b}\left(\theta_{1}, \theta_{2}, \mathbf{y}\right), \mathcal{J}_{\mathbf{\Psi}}^{a}\left(\theta_{1}, \theta_{2}, \mathbf{x}\right)$ and $\mathcal{J}_{\mathbf{\Psi}}^{b}\left(\theta_{1}, \theta_{2}, \mathbf{x}\right)$ the corresponding Jacobian matrices.

We define the difference between the velocity at time $t$ in $\mathscr{F}_{s}$ and the stationary velocity for each of these diffeomorphisms

$$
\forall \mathbf{y} \in \mathscr{F}_{s}, \quad \forall t \in(0, \infty), \quad\left\{\begin{array}{l}
\mathbf{v}^{a}(t, \mathbf{y})=\operatorname{cof}\left(\mathcal{J}_{\mathbf{\Phi}}^{a}\left(\theta_{1}(t), \theta_{2}(t), \mathbf{y}\right)\right)^{T} \mathbf{u}\left(t, \boldsymbol{\Phi}^{a}\left(\theta_{1}(t), \theta_{2}(t), \mathbf{y}\right)\right)-\mathbf{w}(\mathbf{y}) \\
q^{a}(t, \mathbf{y})=p\left(t, \boldsymbol{\Phi}^{a}\left(\theta_{1}, \theta_{2}, \mathbf{y}\right)\right)-p_{\mathbf{w}}(\mathbf{y}) \\
\mathbf{v}^{b}(t, \mathbf{y})=\operatorname{cof}\left(\mathcal{J}_{\mathbf{\Phi}}^{b}\left(\theta_{1}(t), \theta_{2}(t), \mathbf{y}\right)\right)^{T} \mathbf{u}\left(t, \boldsymbol{\Phi}^{b}\left(\theta_{1}(t), \theta_{2}(t), \mathbf{y}\right)\right)-\mathbf{w}(\mathbf{y}) \\
q^{b}(t, \mathbf{y})=p\left(t, \boldsymbol{\Phi}^{b}\left(\theta_{1}, \theta_{2}, \mathbf{y}\right)\right)-p_{\mathbf{w}}(\mathbf{y})
\end{array}\right.
$$

We have the relations

$$
\left\{\begin{array}{c}
\mathbf{v}^{b}(t, \mathbf{y})=\operatorname{cof}\left(\mathcal{J}_{\mathbf{\Phi}}^{b}\left(\theta_{1}, \theta_{2}, \mathbf{y}\right)\right)^{T} \operatorname{cof}\left(\mathcal{J}_{\mathbf{\Psi}}^{a}\left(\theta_{1}, \theta_{2}, \boldsymbol{\Phi}^{b}\left(\theta_{1}, \theta_{2}, \mathbf{y}\right)\right)\right)^{T}\left(\mathbf{v}^{a}\left(t, \mathbf{\Psi}^{a}\left(\theta_{1}, \theta_{2}, \boldsymbol{\Phi}^{b}\left(\theta_{1}, \theta_{2}, \mathbf{y}\right)\right)\right)\right. \\
\left.+\mathbf{w}\left(\mathbf{\Psi}^{a}\left(\theta_{1}, \theta_{2}, \boldsymbol{\Phi}^{b}\left(\theta_{1}, \theta_{2}, \mathbf{y}\right)\right)\right)\right)-\mathbf{w}(\mathbf{y}) \\
q^{b}(t, \mathbf{y})=q^{a}\left(t, \Psi^{a}\left(\theta_{1}, \theta_{2}, \boldsymbol{\Phi}^{b}\left(\theta_{1}, \theta_{2}, \mathbf{y}\right)\right)+p_{\mathbf{w}}\left(\mathbf{\Psi}^{a}\left(\theta_{1}, \theta_{2}, \boldsymbol{\Phi}^{b}\left(\theta_{1}, \theta_{2}, \mathbf{y}\right)\right)\right)-p_{\mathbf{w}}(\mathbf{y})\right.
\end{array}\right.
$$

Of course, $\left(\mathbf{v}^{a}, q^{a}, \theta_{1}, \theta_{2}\right)$ satisfies the nonlinear system (3.2)-(3.3) corresponding to the diffeomorphisms $\boldsymbol{\Phi}^{a}\left(\theta_{1}, \theta_{2},.\right)$ and $\boldsymbol{\Psi}^{a}\left(\theta_{1}, \theta_{2},.\right)$. Similarly, $\left(\mathbf{v}^{b}, q^{b}, \theta_{1}, \theta_{2}\right)$ fulfils $(3.2)-(3.3)$ given by $\boldsymbol{\Phi}^{b}\left(\theta_{1}, \theta_{2},.\right)$ and $\boldsymbol{\Psi}^{b}\left(\theta_{1}, \theta_{2},.\right)$.

We are interested in the following system

$$
\begin{cases}\frac{\partial \mathbf{v}_{L}^{\alpha}}{\partial t}+(\mathbf{w} \cdot \nabla) \mathbf{v}_{L}^{\alpha}+\left(\mathbf{v}_{L}^{\alpha} \cdot \nabla\right) \mathbf{w}-\mathbf{L}_{\mathbf{F}}^{\alpha}\left(\theta_{1}, \theta_{2}, \dot{\theta}_{1}, \dot{\theta}_{2}, \mathbf{y}\right)-\nu \Delta \mathbf{v}_{L}^{\alpha}+\nabla q_{L}^{\alpha}=0 & \text { in }(0, \infty) \times \mathscr{F}_{s}, \\
\operatorname{div} \mathbf{v}_{L}^{\alpha}=0 & \text { in }(0, \infty) \times \mathscr{F}_{s}, \\
\mathbf{v}_{L}^{\alpha}=\dot{\theta}_{1} \partial_{\theta_{1}} \boldsymbol{\Phi}^{\alpha}(0,0, .)+\dot{\theta}_{2} \partial_{\theta_{2}} \boldsymbol{\Phi}^{\alpha}(0,0, .) & \text { on }(0, \infty) \times \partial S_{s}, \\
\mathbf{v}_{L}^{\alpha}=0 & \text { on }(0, \infty) \times \Gamma_{\mathrm{D}}, \\
\sigma_{F}\left(\mathbf{v}_{L}^{\alpha}, q_{L}^{\alpha}\right) \mathbf{n}=0 & \text { on }(0, \infty) \times \Gamma_{\mathrm{N}}, \\
\mathcal{M}_{0,0}\left(\begin{array}{l}
\ddot{\theta}_{1} \\
\ddot{\theta}_{2}
\end{array}\right)=\left(\int_{\partial S_{s}}\left(q_{L}^{\alpha} \mathrm{I}-\nu\left(\nabla \mathbf{v}_{L}^{\alpha}+\left(\nabla \mathbf{v}_{L}^{\alpha}\right)^{T}\right)\right) \mathbf{n}_{s} \cdot \partial_{\theta_{j}} \boldsymbol{\Phi}^{\alpha}\left(0,0, \gamma_{y}\right) \mathrm{d} \gamma_{y}\right)_{j=1,2} & \\
\mathbf{L}_{\mathbf{S}}\left(\theta_{1}, \theta_{2}\right) & \text { on }(0, \infty),\end{cases}
$$

where $\alpha=a$ or $b$, and $\mathbf{L}_{\mathbf{F}}^{\alpha}$ and $\mathbf{L}_{\mathbf{S}}^{\alpha}$ are given respectively by (2.2) and (2.3) corresponding to the choice of diffeomorphism $\boldsymbol{\Phi}\left(\theta_{1}, \theta_{2},.\right)=\boldsymbol{\Phi}^{\alpha}\left(\theta_{1}, \theta_{2},.\right)$.

We can then show that $\left(\mathbf{v}_{L}^{a}, q_{L}^{a}\right)$ fulfils the linear system (B.1) with $\alpha=a$ if and only if

$$
\left\{\begin{aligned}
& \mathbf{v}_{L}^{b}(t, \mathbf{y})=\mathbf{v}_{L}^{a}(t, \mathbf{y})+\sum_{j} \theta_{j}\left(\operatorname{cof}\left(\partial_{\theta_{j}} \mathcal{J}_{\mathbf{\Phi}}^{b}(0,0, \mathbf{y})\right)^{T} \mathbf{w}(\mathbf{y})+\operatorname{cof}\left(\partial_{\theta_{j}} \mathcal{J}_{\mathbf{\Psi}}^{a}(0,0, \mathbf{y})\right)^{T} \mathbf{w}(\mathbf{y})\right.\left.+\nabla \mathbf{w} \times\left(\partial_{\theta_{j}} \mathbf{\Psi}^{a}(0,0, \mathbf{y})+\partial_{\theta_{j}} \boldsymbol{\Phi}^{b}(0,0, \mathbf{y})\right)\right) \\
& q_{L}^{b}(t, \mathbf{y})=q_{L}^{a}(t, \mathbf{y})+\sum_{j} \theta_{j} \nabla p_{\mathbf{w}} \cdot\left(\partial_{\theta_{j}} \boldsymbol{\Phi}^{a}(0,0, \mathbf{y})-\partial_{\theta_{j}} \boldsymbol{\Phi}^{b}(0,0, \mathbf{y})\right)
\end{aligned}\right.
$$

fulfils the linear system (B.1) with $\alpha=b$. 
The proof is a direct computation, for instance

$$
\begin{aligned}
\frac{\partial \mathbf{v}_{L}^{b}}{\partial t}-\mathbf{L}_{3}^{b} \dot{\theta}_{1}-\mathbf{L}_{4}^{b} \dot{\theta}_{2} & =\frac{\partial \mathbf{v}_{L}^{a}}{\partial t}+\sum_{j} \dot{\theta}_{j}\left(\operatorname{cof}\left(\partial_{\theta_{j}} \mathcal{J}_{\mathbf{\Phi}}^{b}(0,0, \mathbf{y})-\partial_{\theta_{j}} \mathcal{J}_{\mathbf{\Phi}}^{a}(0,0, \mathbf{y})\right)^{T} \mathbf{w}+\nabla \mathbf{w} \times\left(\partial_{\theta_{j}} \boldsymbol{\Phi}^{b}(0,0, \mathbf{y})-\partial_{\theta_{j}} \boldsymbol{\Phi}^{a}(0,0, \mathbf{y})\right)\right) \\
& =\frac{\partial \mathbf{v}_{L}^{a}}{\partial t}-\mathbf{L}_{3}^{b} \dot{\theta}_{1}-\mathbf{L}_{4}^{b} \dot{\theta}_{1}-\mathbf{L}_{4}^{a} \dot{\theta}_{2}
\end{aligned}
$$

Moreover by using $\operatorname{div} \mathbf{w}=0, \operatorname{cof}\left(\partial_{\theta_{k}} \mathcal{J}_{\boldsymbol{\Phi}}(0,0, \mathbf{y})\right)^{T}: \nabla \mathbf{w}=\partial_{\theta_{k}} \mathcal{J}_{\boldsymbol{\Psi}}(0,0, \mathbf{y}): \nabla \mathbf{w}=-\partial_{\theta_{k}} \mathcal{J}_{\boldsymbol{\Phi}}(0,0, \mathbf{y}): \nabla \mathbf{w}$ and the Piola identity, we get

$$
\operatorname{div} \mathbf{v}_{L}^{b}=\operatorname{div} \mathbf{v}_{L}^{a}=0 .
$$

This implies that the solution $\left(\mathbf{v}_{L}^{b}, q_{L}^{b}, \theta_{1}, \theta_{2}\right)$ to the system (B.1) written with the diffeomorphism $\boldsymbol{\Phi}^{b}$ can be derived from the solution to the system (B.1) written with the diffeomorphism $\boldsymbol{\Phi}^{a}$ via the relation (B.2). Then the stabilizability of (2.1) is independent with respect to the choice of the diffeomorphism $\boldsymbol{\Phi}$. As the Hautus test $(\mathcal{H})_{\delta}$ is equivalent to the stabilizability of $(2.1)$, the hypothesis $(\mathcal{H})_{\delta}$ is independent from the choice of $\boldsymbol{\Phi}$.

\section{Proof of Lemma 3.2}

We first prove some technical lemmas that are used later to decompose the intricate terms of Lemma 3.2.

\section{C.1 Technical lemmas}

Lemma C.1. Let $R_{0}>0$ be small enough, then there exists a constant $K$, such that for every $R \leq R_{0}$ and every $\left(., ., \theta_{1}^{j}, \theta_{2}^{j}\right)$ in $\widetilde{\mathrm{B}_{R}^{\infty}}$, the following estimates hold

$$
\begin{aligned}
& \left\|e^{\delta t}\left(\mathbf{\Phi}\left(\theta_{1}^{a}, \theta_{2}^{a}\right)-\mathbf{\Phi}\left(\theta_{1}^{b}, \theta_{2}^{b}\right)-\sum_{n}\left(\theta_{n}^{a}-\theta_{n}^{b}\right) \partial_{\theta_{n}} \mathbf{\Phi}(0,0, .)\right)\right\|_{L^{\infty}\left(0, \infty ; \mathbf{H}^{3}(\Omega)\right)} \leq K R\left\|\theta^{a}-\theta^{b}\right\|_{\Theta_{\delta}^{\infty}}, \\
& \left\|e^{\delta t}\left(\mathcal{J}_{\mathbf{\Phi}}\left(\theta_{1}^{a}, \theta_{2}^{a}\right)-\mathcal{J}_{\boldsymbol{\Phi}}\left(\theta_{1}^{b}, \theta_{2}^{b}\right)-\sum_{n}\left(\theta_{n}^{a}-\theta_{n}^{b}\right) \nabla_{\mathbf{y}} \partial_{\theta_{n}} \mathbf{\Phi}(0,0, .)\right)\right\|_{\mathrm{L}^{\infty}\left(0, \infty ; \mathbf{H}^{2}(\Omega)\right)} \leq K R\left\|\theta^{a}-\theta^{b}\right\|_{\Theta_{\delta}^{\infty}} \\
& \| e^{\delta t}\left(\mathcal{J}_{\mathbf{\Psi}}\left(\theta_{1}^{a}, \theta_{2}^{a}\right) \circ \mathbf{\Phi}\left(\theta_{1}^{a}, \theta_{2}^{a}\right)-\mathcal{J}_{\mathbf{\Psi}}\left(\theta_{1}^{b}, \theta_{2}^{b}\right) \circ \mathbf{\Phi}\left(\theta_{1}^{b}, \theta_{2}^{b}\right)\right. \\
& \left.+\sum_{j}\left(\theta_{j}^{a}-\theta_{j}^{b}\right) \nabla_{\mathbf{y}} \partial_{\theta_{j}} \mathbf{\Phi}(0,0, .)\right)\left\|_{L^{\infty}\left(0, \infty ; \mathbf{H}^{2}(\Omega)\right)} \leq K R\right\| \theta^{a}-\theta^{b} \|_{\Theta_{\delta}^{\infty}}, \\
& \| e^{\delta t}\left(\partial_{x_{j}} \mathcal{J}_{\Psi}\left(\theta_{1}^{a}, \theta_{2}^{a}\right) \circ \mathbf{\Phi}\left(\theta_{1}^{a}, \theta_{2}^{a}\right)-\partial_{x_{j}} \mathcal{J}_{\Psi}\left(\theta_{1}^{b}, \theta_{2}^{b}\right) \circ \boldsymbol{\Phi}\left(\theta_{1}^{b}, \theta_{2}^{b}\right)\right. \\
& \left.-\sum_{n}\left(\theta_{n}^{a}-\theta_{n}^{b}\right) \partial_{x_{j}} \nabla_{\mathbf{x}} \partial_{\theta_{n}} \mathbf{\Psi}(0,0, .)\right)\left\|_{L^{\infty}\left(0, \infty ; \mathbf{H}^{1}(\Omega)\right)} \leq K R\right\| \theta^{a}-\theta^{b} \|_{\Theta_{\delta}^{\infty}}, \\
& \| e^{\delta t}\left(\partial_{x_{j}}^{2} \mathcal{J}_{\Psi}\left(\theta_{1}^{a}, \theta_{2}^{a}\right) \circ \boldsymbol{\Phi}\left(\theta_{1}^{a}, \theta_{2}^{a}\right)-\partial_{x_{j}}^{2} \mathcal{J}_{\Psi}\left(\theta_{1}^{b}, \theta_{2}^{b}\right) \circ \boldsymbol{\Phi}\left(\theta_{1}^{b}, \theta_{2}^{b}\right)\right. \\
& \left.-\sum_{n}\left(\theta_{n}^{a}-\theta_{n}^{b}\right) \partial_{x_{j}}^{2} \nabla_{\mathbf{x}} \partial_{\theta_{n}} \mathbf{\Psi}(0,0, .)\right)\left\|_{L^{\infty}\left(0, \infty ; \mathbf{L}^{2}(\Omega)\right)} \leq K R\right\| \theta^{a}-\theta^{b} \|_{\Theta_{\delta}^{\infty}}, \\
& \left\|e^{\delta t}\left(\mathbf{n}_{\theta_{1}^{a}, \theta_{2}^{a}}\left(\mathbf{\Phi}\left(\theta_{1}^{a}, \theta_{2}^{a}\right)\right)_{j}-\mathbf{n}_{\theta_{1}^{b}, \theta_{2}^{b}}\left(\mathbf{\Phi}\left(\theta_{1}^{b}, \theta_{2}^{b}\right)\right)_{j}-\sum_{n}\left(\theta_{n}^{a}-\theta_{n}^{b}\right)\left(\mathbf{L}_{\mathbf{n}_{\theta_{1}, \theta_{2}}}\right)_{j, n}\right)\right\|_{L^{\infty}\left(0, \infty ; L^{\infty}\left(\partial S_{s}\right)\right)} \\
& \leq K R\left\|\theta^{a}-\theta^{b}\right\|_{\Theta_{\delta}^{\infty}}, \\
& \| e^{\delta t}\left(\operatorname{det}\left(\mathcal{J}_{\mathbf{\Psi}}\left(\theta_{1}^{a}, \theta_{2}^{a}, \boldsymbol{\Phi}^{a}\right)\right)-\operatorname{det}\left(\mathcal{J}_{\mathbf{\Psi}}\left(\theta_{1}^{b}, \theta_{2}^{b}, \boldsymbol{\Phi}^{b}\right)\right)\right. \\
& \left.+\sum_{n}\left(\theta_{n}^{a}-\theta_{n}^{b}\right) \operatorname{Tr}\left(\nabla_{\mathbf{y}} \partial_{\theta_{n}} \mathbf{\Phi}(0,0, .)\right)\right)\left\|_{\mathrm{L}^{\infty}\left(0, \infty ; \mathbf{L}^{\infty}(\Omega)\right)} \leq K R\right\| \theta^{a}-\theta^{b} \|_{\Theta_{\delta}^{\infty}}, \\
& \left\|e^{\delta t}\left(\left|\mathcal{J}_{\mathbf{\Phi}}\left(\theta_{1}^{a}, \theta_{2}^{a}\right) \mathbf{t}_{s}\right|-\left|\mathcal{J}_{\mathbf{\Phi}}\left(\theta_{1}^{b}, \theta_{2}^{b}\right) \mathbf{t}_{s}\right|-\sum_{k}\left(\theta_{k}^{a}-\theta_{k}^{b}\right) \nabla_{\mathbf{y}} \partial_{\theta_{k}} \mathbf{\Phi}(0,0, .) \mathbf{t}_{s} \cdot \mathbf{t}_{s}\right)\right\|_{\mathrm{L}^{\infty}\left(0, \infty ; \mathbf{L}^{\infty}\left(\partial S_{s}\right)\right)} \\
& \leq K R\left\|\theta^{a}-\theta^{b}\right\|_{\Theta_{\delta}^{\infty}}
\end{aligned}
$$


where $\mathbf{L}_{\mathbf{n}_{\theta_{1}, \theta_{2}}}$ is defined in (A.11) and

$$
\begin{aligned}
& \| e^{\delta t}\left(\left(\partial_{t} \mathbf{\Psi}\left(\theta_{1}^{a}, \theta_{2}^{a}\right)\right) \circ \mathbf{\Phi}\left(\theta_{1}^{a}, \theta_{2}^{a}\right)-\left(\partial_{t} \mathbf{\Psi}\left(\theta_{1}^{b}, \theta_{2}^{b}\right)\right) \circ \mathbf{\Phi}\left(\theta_{1}^{b}, \theta_{2}^{b}\right)\right. \\
& \left.+\sum_{j}\left(\dot{\theta}_{j}^{a}-\dot{\theta}_{j}^{b}\right) \partial_{\theta_{j}} \boldsymbol{\Phi}(0,0, .)\right)\left\|_{L^{\infty}\left(0, \infty ; \mathbf{L}^{\infty}(\Omega)\right)} \leq K R\right\| \theta^{a}-\theta^{b} \|_{\Theta_{\delta}^{\infty}}, \\
& \| e^{\delta t}\left(\partial_{t}\left(\mathcal{J}_{\Psi}\left(\theta_{1}^{a}, \theta_{2}^{a}\right)\right) \circ \mathbf{\Phi}\left(\theta_{1}^{a}, \theta_{2}^{a}\right)-\partial_{t}\left(\mathcal{J}_{\Psi}\left(\theta_{1}^{b}, \theta_{2}^{b}\right)\right) \circ \mathbf{\Phi}\left(\theta_{1}^{b}, \theta_{2}^{b}\right)\right. \\
& \left.+\sum_{j}\left(\dot{\theta}_{j}^{a}-\dot{\theta}_{j}^{b}\right) \nabla_{\mathbf{y}} \partial_{\theta_{j}} \boldsymbol{\Phi}(0,0, .)\right)\left\|_{L^{\infty}\left(0, \infty ; \mathbf{L}^{\infty}(\Omega)\right)} \leq K R\right\| \theta^{a}-\theta^{b} \|_{\Theta_{\delta}^{\infty}},
\end{aligned}
$$

and

$$
\begin{aligned}
&\left\|e^{\delta t}\left(\mathcal{M}_{\theta_{1}^{a}, \theta_{2}^{a}}-\mathcal{M}_{\theta_{1}^{b}, \theta_{2}^{b}}\right)\right\|_{L^{\infty}(0, \infty)} \leq K\left\|\theta^{a}-\theta^{b}\right\|_{\Theta_{\delta}^{\infty}}, \\
&\left\|e^{\delta t}\left(\partial_{\theta_{j}} \boldsymbol{\Phi}\left(\theta_{1}^{a}, \theta_{2}^{a}, .\right)-\partial_{\theta_{j}} \mathbf{\Phi}\left(\theta_{1}^{b}, \theta_{2}^{b}, .\right)-\sum_{k}\left(\theta_{k}^{a}-\theta_{k}^{b}\right) \partial_{\theta_{j} \theta_{k}} \mathbf{\Phi}(0,0, .)\right)\right\|_{L^{\infty}\left(0, \infty ; \mathbf{H}^{2}(\Omega)\right)} \leq K\left\|\theta^{a}-\theta^{b}\right\|_{\Theta_{\delta}^{\infty}}, \\
&\left\|e^{\delta t}\left(\partial_{\theta_{j} \theta_{k}} \mathbf{\Phi}\left(\theta_{1}^{a}, \theta_{2}^{a}, .\right)-\partial_{\theta_{j} \theta_{k}} \mathbf{\Phi}\left(\theta_{1}^{b}, \theta_{2}^{b}, .\right)\right)\right\|_{L_{\left(0, \infty ; \mathbf{H}^{2}(\Omega)\right)}} \leq K\left\|\theta^{a}-\theta^{b}\right\|_{\Theta_{\delta}^{\infty}}, \\
&\left\|e^{\delta t}\left(\partial_{t} \mathcal{J}_{\boldsymbol{\Phi}}\left(\theta_{1}^{a}, \theta_{2}^{a}\right)-\partial_{t} \mathcal{J}_{\boldsymbol{\Phi}}\left(\theta_{1}^{b}, \theta_{2}^{b}\right)\right)\right\|_{L^{\infty}\left(0, \infty ; \mathbf{H}^{2}(\Omega)\right)} \leq K\left\|\theta^{a}-\theta^{b}\right\|_{\Theta_{\delta}^{\infty}} .
\end{aligned}
$$

Moreover for every $\left(\mathbf{v}^{j}, ., \theta_{1}^{j}, \theta_{2}^{j}\right)$ in $\widetilde{\mathbb{B}_{R}^{\infty}}$, we have

$$
\begin{array}{r}
\left\|e^{\delta t}\left(\nabla \mathbf{v}^{a}-\mathcal{G}\left(\theta_{1}^{a}, \theta_{2}^{a}, \mathbf{v}^{a}\right)-\nabla \mathbf{v}^{b}+\mathcal{G}\left(\theta_{1}^{b}, \theta_{2}^{b}, \mathbf{v}^{b}\right)\right)\right\|_{L^{2}\left(0, \infty ; \mathbf{L}^{2}\left(\partial S_{s}\right)\right)} \leq K R\left(\left\|\theta^{a}-\theta^{b}\right\|_{\Theta_{\delta}^{\infty}}+\left\|\mathbf{v}^{a}-\mathbf{v}^{b}\right\|_{\mathrm{U}_{\delta}^{\infty}}\right), \\
\left\|e^{\delta t}\left(\mathcal{G}\left(\theta_{1}^{a}, \theta_{2}^{a}, \mathbf{w}\right)_{i j}-\mathcal{G}\left(\theta_{1}^{b}, \theta_{2}^{b}, \mathbf{w}\right)_{i j}-\sum_{n}\left(\theta_{n}^{a}-\theta_{n}^{b}\right)\left(\mathbf{L}_{\mathcal{G}}\right)_{i, j, n}\right)\right\|_{L^{2}\left(0,+\infty ; L^{2}\left(\partial S_{s}\right)\right)} \leq K R\left\|\theta^{a}-\theta^{b}\right\|_{\Theta_{\delta}^{\infty}},
\end{array}
$$

where $\mathbf{L}_{\mathcal{G}}$ is defined in (A.12) and $\mathcal{G}$ is defined in (3.7).

A direct application of Lemma C.1 obtained by taking $\left(\theta_{1}^{b}, \theta_{2}^{b}\right)=(0,0)$ is the following lemma.

Lemma C.2. Let $R_{0}>0$ be small enough, then there exists $K>0$ such that for every $R \leq R_{0}$ and for every $\left(., ., \theta_{1}, \theta_{2}\right) \in \widetilde{\mathbb{B}_{R}^{\infty}}$, the following estimates hold

$$
\begin{aligned}
& \left\|e^{\delta t}\left(\mathcal{J}_{\boldsymbol{\Phi}}\left(\theta_{1}, \theta_{2}\right)-\mathrm{I}\right)\right\|_{\mathrm{L}^{\infty}\left(0, \infty ; \mathbf{H}^{2}(\Omega)\right)} \leq K R, \\
& \left\|e^{\delta t}\left(\mathcal{J}_{\Psi}\left(\theta_{1}, \theta_{2}, \mathbf{\Phi}\left(\theta_{1}, \theta_{2}\right)\right)-\mathrm{I}\right)\right\|_{\mathrm{L}^{\infty}\left(0, \infty ; \mathbf{H}^{2}(\Omega)\right)} \leq K R, \\
& \left\|e^{\delta t}\left(\partial_{x_{j}} \mathcal{J}_{\Psi}\left(\theta_{1}, \theta_{2}\right) \circ \mathbf{\Phi}\left(\theta_{1}, \theta_{2}\right)\right)\right\|_{L^{\infty}\left(0, \infty ; \mathbf{H}^{1}(\Omega)\right)} \leq K R, \\
& \left\|e^{\delta t}\left(\partial_{x_{j}}^{2} \mathcal{J}_{\Psi}\left(\theta_{1}, \theta_{2}\right) \circ \mathbf{\Phi}\left(\theta_{1}, \theta_{2}\right)\right)\right\|_{L^{\infty}\left(0, \infty ; \mathbf{L}^{2}(\Omega)\right)} \leq K R, \\
& \left\|e^{\delta t}\left(\mathcal{M}_{\theta_{1}, \theta_{2}}-\mathcal{M}_{0,0}\right)\right\|_{L^{\infty}(0, \infty)} \leq K R, \\
& \left\|e^{\delta t}\left(\mathbf{n}_{\theta_{1}, \theta_{2}}\left(\mathbf{\Phi}\left(\theta_{1}, \theta_{2}\right)\right)-\mathbf{n}_{s}\right)\right\|_{L^{\infty}\left(0, \infty ; \mathbf{L}^{\infty}\left(\partial S_{s}\right)\right)} \leq K R,
\end{aligned}
$$

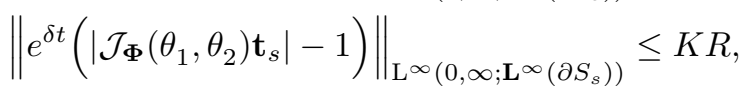

$$
\begin{aligned}
& \left\|e^{\delta t}\left(\partial_{\theta_{j}} \mathbf{\Phi}\left(\theta_{1}, \theta_{2}, .\right)-\partial_{\theta_{j}} \mathbf{\Phi}(0,0, .)\right)\right\|_{L^{\infty}\left(0, \infty ; \mathbf{H}^{3}(\Omega)\right)} \leq K R,
\end{aligned}
$$

and

$$
\begin{aligned}
&\left\|e^{\delta t} \partial_{t} \mathcal{J}_{\mathbf{\Phi}}\left(\theta_{1}, \theta_{2}\right)\right\|_{\mathrm{L}^{\infty}\left(0, \infty ; \mathbf{H}^{2}(\Omega)\right)} \leq K R, \\
&\left\|e^{\delta t}\left(\partial_{t} \mathbf{\Psi}\left(\theta_{1}, \theta_{2}\right)\right) \circ \boldsymbol{\Phi}\left(\theta_{1}, \theta_{2}\right)\right\|_{\mathrm{L}^{\infty}\left(0, \infty ; \mathbf{L}^{\infty}(\Omega)\right)} \leq K R, \\
&\left\|e^{\delta t}\left(\partial_{t} \mathcal{J}_{\Psi}\left(\theta_{1}, \theta_{2}\right)\right) \circ \mathbf{\Phi}\left(\theta_{1}, \theta_{2}\right)\right\|_{\mathrm{L}^{\infty}\left(0, \infty ; \mathbf{L}^{\infty}(\Omega)\right)} \leq K R,
\end{aligned}
$$

and

$$
\left\|e^{\delta t}\left(\nabla \mathbf{w}-\mathcal{G}\left(\theta_{1}, \theta_{2}, \mathbf{w}\right)\right)\right\|_{L^{2}\left(0, \infty ; \mathbf{L}^{2}\left(\partial S_{s}\right)\right)} \leq K R,
$$

where $\mathcal{G}$ is defined in (3.7). 
Proof of Lemma C.1. Let $\mathbb{X}$ be some Banach space. All functions in Lemma C.1 can be written as $\alpha=$ $\alpha\left(\theta_{1}, \theta_{2}, \dot{\theta}_{1}, \dot{\theta}_{2}\right)$ valued in $\mathbb{X}$. The proof of estimates (C.11)-(C.14) uses the mean value theorem,

$$
\begin{aligned}
\| e^{\delta t}\left(\alpha\left(\theta_{1}^{a}, \theta_{2}^{a}, \dot{\theta}_{1}^{a}, \dot{\theta}_{2}^{a}\right)\right. & \left.-\alpha\left(\theta_{1}^{b}, \theta_{2}^{b}, \dot{\theta}_{1}^{b}, \dot{\theta}_{2}^{b}\right)\right) \|_{\mathrm{L}^{\infty}(0, \infty ; \mathbb{X})} \\
\leq & \sup _{\substack{\left(\theta_{1}, \theta_{2}\right) \in \mathbb{D}_{\Theta} \\
\left|\omega_{1}\right|+\left|\omega_{2}\right| \leq R}}\left\|\nabla_{\theta, \omega} \alpha\left(\theta_{1}, \theta_{2}, \omega_{1}, \omega_{2}\right)\right\|_{L^{\infty}(0, \infty ; \mathbb{X})}\left(\left\|e^{\delta t}\left(\theta^{a}-\theta^{b}\right)\right\|_{L^{\infty}(0, \infty)}+\left\|e^{\delta t}\left(\dot{\theta}^{a}-\dot{\theta}^{b}\right)\right\|_{L^{\infty}(0, \infty)}\right) \\
\leq & \underset{\substack{\left(\theta_{1}, \theta_{2}\right) \in \mathbb{D}_{\Theta} \\
\left|\omega_{1}\right|+\left|\omega_{2}\right| \leq R}}{2 C \sup _{\theta, \omega} \alpha\left(\theta_{1}, \theta_{2}, \omega_{1}, \omega_{2}\right)\left\|_{L^{\infty}(0, \infty ; \mathbb{X})}\right\| \theta^{a}-\theta^{b} \|_{\Theta_{\delta}^{\infty}} .}
\end{aligned}
$$

To prove the other estimates (C.1)-(C.10) and (C.16), we do a Taylor expansion of order 2 and use the mean value theorem. We get

$$
\begin{gathered}
\left\|e^{\delta t}\left(\alpha\left(\theta_{1}^{a}, \theta_{2}^{a}, \dot{\theta}_{1}^{a}, \dot{\theta}_{2}^{a}\right)-\alpha\left(\theta_{1}^{b}, \theta_{2}^{b}, \dot{\theta}_{1}^{b}, \dot{\theta}_{2}^{b}\right)-\sum_{j=1}^{2} \partial_{\theta_{j}} \alpha(0,0,0,0)\left(\theta_{j}^{a}-\theta_{j}^{b}\right)-\sum_{j=1}^{2} \partial_{\omega_{j}} \alpha(0,0,0,0)\left(\dot{\theta}_{j}^{a}-\dot{\theta}_{j}^{b}\right)\right)\right\|_{L^{\infty}(0, \infty ; \mathbb{X})} \\
\leq \sup _{\substack{\left(\theta_{1}, \theta_{2}\right) \in \mathbb{D}_{\Theta} \\
\left|\omega_{1}\right|+\left|\omega_{2}\right| \leq R}}\left\|\nabla_{\theta, \omega}^{2} \alpha\left(\theta_{1}, \theta_{2}, \omega_{1}, \omega_{2}\right)\right\|_{L_{(0, \infty ; \mathbb{X})} R\left(\left\|e^{\delta t}\left(\theta^{a}-\theta^{b}\right)\right\|_{L^{\infty}(0, \infty)}+\left\|e^{\delta t}\left(\dot{\theta}^{a}-\dot{\theta}^{b}\right)\right\|_{L^{\infty}(0, \infty)}\right) .}
\end{gathered}
$$

The estimate (C.15) is proven by using the previous estimates.

\section{C.2 Detailed proof of Lemma 3.2}

Proof. The weights in time $e^{\delta t}$ of the nonlinear terms can be easily handled. All the difficulties then come from space regularity issues, which can be handled as in [11, Appendix B] for the terms $\mathbf{F}\left(\theta_{1}^{a}, \theta_{2}^{a}, \mathbf{v}^{a}, q^{a}\right)-$ $\mathbf{F}\left(\theta_{1}^{b}, \theta_{2}^{b}, \mathbf{v}^{b}, q^{b}\right), \mathbf{G}\left(\theta_{1}^{a}, \theta_{2}^{a}, \dot{\theta}_{1}^{a}, \dot{\theta}_{2}^{a}\right)-\mathbf{G}\left(\theta_{1}^{b}, \theta_{2}^{b}, \dot{\theta}_{1}^{b}, \dot{\theta}_{2}^{b}\right)$ and $\mathbf{S}\left(\theta_{1}^{a}, \theta_{2}^{a}, \mathbf{v}^{a}, q^{a}\right)-\mathbf{S}\left(\theta_{1}^{b}, \theta_{2}^{b}, \mathbf{v}^{b}, q^{b}\right)$ using the estimates of Lammas C.1 and C.2:

$$
\left\|\mathbf{F}\left(\theta_{1}^{a}, \theta_{2}^{a}, \mathbf{v}^{a}, q^{a}\right)-\mathbf{F}\left(\theta_{1}^{b}, \theta_{2}^{b}, \mathbf{v}^{b}, q^{b}\right)\right\|_{\mathbb{F}_{\delta}^{\infty}} \leq K R\left(\left\|\mathbf{v}^{a}-\mathbf{v}^{b}\right\|_{\mathbb{U}_{\delta}^{\infty}}+\left\|q^{a}-q^{b}\right\|_{\mathbb{P}_{\delta}^{\infty}}+\left\|\theta^{a}-\theta^{b}\right\|_{\Theta_{\delta}^{\infty}}\right),
$$

$$
\left\|\mathbf{G}\left(\theta_{1}^{a}, \theta_{2}^{a}, \dot{\theta}_{1}^{a}, \dot{\theta}_{2}^{a}\right)-\mathbf{G}\left(\theta_{1}^{b}, \theta_{2}^{b}, \dot{\theta}_{1}^{b}, \dot{\theta}_{2}^{b}\right)\right\|_{\mathbb{G}_{\delta}^{\infty}} \leq K R\left\|\theta^{a}-\theta^{b}\right\|_{\Theta_{\delta}^{\infty}}
$$

and

$$
\left\|\mathbf{S}\left(\theta_{1}^{a}, \theta_{2}^{a}, \mathbf{v}^{a}, q^{a}\right)-\mathbf{S}\left(\theta_{1}^{b}, \theta_{2}^{b}, \mathbf{v}^{b}, q^{b}\right)\right\|_{\mathbb{S}_{\delta}^{\infty}} \leq K R\left(\left\|\mathbf{v}^{a}-\mathbf{v}^{b}\right\|_{\mathbf{U}_{\delta}^{\infty}}+\left\|q^{a}-q^{b}\right\|_{\mathbb{P}_{\delta}^{\infty}}+\left\|\theta^{a}-\theta^{b}\right\|_{\Theta_{\delta}^{\infty}}\right),
$$

where the terms $\mathbf{F}, \mathbf{G}$ and $\mathbf{S}$ are defined in (3.5)-(3.6).

Now, it remains to prove

$$
\begin{gathered}
\| \mathbf{F}\left(\theta_{1}^{a}, \theta_{2}^{a}, \mathbf{w}+\mathbf{v}^{a}, p_{\mathbf{w}}+q^{a}\right)-\mathbf{F}\left(\theta_{1}^{b}, \theta_{2}^{b}, \mathbf{w}+\mathbf{v}^{b}, p_{\mathbf{w}}+q^{b}\right)-\mathbf{F}\left(\theta_{1}^{a}, \theta_{2}^{a}, \mathbf{v}^{a}, q^{a}\right)+\mathbf{F}\left(\theta_{1}^{b}, \theta_{2}^{b}, \mathbf{v}^{b}, q^{b}\right) \\
+(\mathbf{w} \cdot \nabla)\left(\mathbf{v}^{a}-\mathbf{v}^{b}\right)+\left(\left(\mathbf{v}^{a}-\mathbf{v}^{b}\right) \cdot \nabla\right) \mathbf{w}-\mathbf{L}_{\mathbf{F}}\left(\theta_{1}^{a}-\theta_{1}^{b}, \theta_{2}^{a}-\theta_{2}^{b}, \dot{\theta}_{1}^{a}-\dot{\theta}_{1}^{b}, \dot{\theta}_{2}^{a}-\dot{\theta}_{2}^{b}\right)+\mathbf{f}_{\mathscr{F}}\left(\mathbf{\Phi}\left(\theta_{1}^{a}, \theta_{2}^{a}\right)\right)-\mathbf{f}_{\mathscr{F}}\left(\mathbf{\Phi}\left(\theta_{1}^{b}, \theta_{2}^{b}\right)\right) \|_{\mathbb{F}_{\delta}^{\infty}} \\
\leq C R\left(\left\|\mathbf{v}^{a}-\mathbf{v}^{b}\right\|_{\mathbf{U}_{\delta}^{\infty}}+\left\|\theta^{a}-\theta^{b}\right\|_{\Theta_{\delta}^{\infty}}\right)
\end{gathered}
$$

and

$$
\begin{array}{r}
\| \mathbf{S}\left(\theta_{1}^{a}, \theta_{2}^{a}, \mathbf{w}+\mathbf{v}^{a}, p_{\mathbf{w}}+q^{a}\right)-\mathbf{S}\left(\theta_{1}^{b}, \theta_{2}^{b}, \mathbf{w}+\mathbf{v}^{b}, p_{\mathbf{w}}+q^{b}\right)-\mathbf{S}\left(\theta_{1}^{a}, \theta_{2}^{a}, \mathbf{v}^{a}, q^{a}\right)+\mathbf{S}\left(\theta_{1}^{b}, \theta_{2}^{b}, \mathbf{v}^{b}, q^{b}\right) \\
-\mathbf{L}_{\mathbf{S}}\left(\theta_{1}^{a}-\theta_{1}^{b}, \theta_{2}^{a}-\theta_{2}^{b}\right)\left\|_{\mathbb{S}_{T}} \leq C R\right\| \theta^{a}-\theta^{b} \|_{\Theta_{\delta}^{\infty}},
\end{array}
$$

and then the proof will be complete.

The estimate (C.29) is a consequence of the following relations

$$
\begin{gathered}
\mathbf{F}^{1}\left(\theta_{1}^{a}, \theta_{2}^{a}, \mathbf{w}+\mathbf{v}^{a}\right)-\mathbf{F}^{1}\left(\theta_{1}^{b}, \theta_{2}^{b}, \mathbf{w}+\mathbf{v}^{b}\right)-\mathbf{F}^{1}\left(\theta_{1}^{a}, \theta_{2}^{a}, \mathbf{v}^{a}\right)+\mathbf{F}^{1}\left(\theta_{1}^{b}, \theta_{2}^{b}, \mathbf{v}^{b}\right)=0 \\
\| \mathbf{F}^{2}\left(\theta_{1}^{a}, \theta_{2}^{a}, \mathbf{w}+\mathbf{v}^{a}\right)-\mathbf{F}^{2}\left(\theta_{1}^{b}, \theta_{2}^{b}, \mathbf{w}+\mathbf{v}^{b}\right)-\mathbf{F}^{2}\left(\theta_{1}^{a}, \theta_{2}^{a}, \mathbf{v}^{a}\right)+\mathbf{F}^{2}\left(\theta_{1}^{b}, \theta_{2}^{b}, \mathbf{v}^{b}\right) \\
-\sum_{j}\left(\dot{\theta}_{j}^{a}-\dot{\theta}_{j}^{b}\right)\left(\left(\partial_{\theta_{j}} \mathbf{\Phi}(0,0, \mathbf{y}) \cdot \nabla_{\mathbf{y}}\right) \mathbf{w}+\operatorname{cof}\left(\nabla_{\mathbf{y}} \partial_{\theta_{j}} \mathbf{\Phi}(0,0, \mathbf{y})\right)^{T} \mathbf{w}\right) \|_{\mathbb{F}_{\delta}^{\infty} \leq K R\left\|\theta^{a}-\theta^{b}\right\|_{\Theta_{\delta}^{\infty}}} \\
\| \begin{array}{c}
\left(\mathbf{F}^{3}\left(\theta_{1}^{a}, \theta_{2}^{a}, \mathbf{w}+\mathbf{v}^{a}\right)-\mathbf{F}^{3}\left(\theta_{1}^{b}, \theta_{2}^{b}, \mathbf{w}+\mathbf{v}^{b}\right)-\mathbf{F}^{3}\left(\theta_{1}^{a}, \theta_{2}^{a}, \mathbf{v}^{a}\right)+\mathbf{F}^{3}\left(\theta_{1}^{b}, \theta_{2}^{b}, \mathbf{v}^{b}\right)\right)_{i}-\nu \sum_{j}\left(\theta_{j}^{a}-\theta_{j}^{b}\right)\left(\mathbf{L}_{\mathbf{F}^{3}}\right)_{i j} \|_{\mathbb{F}_{\delta}^{\infty}} \\
\leq K R\left\|\theta^{a}-\theta^{b}\right\|_{\Theta_{\delta}^{\infty}}
\end{array}
\end{gathered}
$$


$\|\left(\mathbf{F}^{4}\left(\theta_{1}^{a}, \theta_{2}^{a}, \mathbf{w}+\mathbf{v}^{a}\right)-\mathbf{F}^{4}\left(\theta_{1}^{a}, \theta_{2}^{a}, \mathbf{v}^{a}\right)-\mathbf{F}^{4}\left(\theta_{1}^{b}, \theta_{2}^{b}, \mathbf{w}+\mathbf{v}^{b}\right)+\mathbf{F}^{4}\left(\theta_{1}^{b}, \theta_{2}^{b}, \mathbf{v}^{b}\right)+\left(\left(\mathbf{v}^{a}-\mathbf{v}^{b}\right) \cdot \nabla\right) \mathbf{w}\right.$

$$
\left.+(\mathbf{w} \cdot \nabla)\left(\mathbf{v}^{a}-\mathbf{v}^{b}\right)\right)_{i}-\sum_{n}\left(\theta_{n}^{a}-\theta_{n}^{b}\right)\left(\mathbf{L}_{\mathbf{F}^{4}}\right)_{i, n} \|_{\mathbb{F}_{\delta}^{\infty}} \leq K R\left(\left\|\mathbf{v}^{a}-\mathbf{v}^{b}\right\|_{\tilde{U}_{\delta}^{\infty}}+\left\|\theta^{a}-\theta^{b}\right\|_{\Theta_{\delta}^{\infty}}\right),
$$

$\| \mathbf{F}^{5}\left(\theta_{1}^{a}, \theta_{2}^{a}, p_{\mathbf{w}}+q^{a}\right)-\mathbf{F}^{5}\left(\theta_{1}^{a}, \theta_{2}^{a}, q^{a}\right)-\mathbf{F}^{5}\left(\theta_{1}^{b}, \theta_{2}^{b}, p_{\mathbf{w}}+q^{b}\right)+\mathbf{F}^{5}\left(\theta_{1}^{b}, \theta_{2}^{b}, q^{b}\right)$

$$
-\sum_{j}\left(\theta_{j}^{a}-\theta_{j}^{b}\right)\left(\nabla_{\mathbf{y}} \partial_{\theta_{j}} \mathbf{\Phi}(0,0, \mathbf{y})\right)^{T} \nabla_{\mathbf{y}} p_{\mathbf{w}}\left\|_{\mathbf{F}_{\delta}^{\infty}} \leq K R\right\| \theta^{a}-\theta^{b} \|_{\Theta_{\delta}^{\infty}},
$$

and

$$
\left\|\mathbf{f}_{\mathscr{F}}\left(\mathbf{\Phi}\left(\theta_{1}^{a}, \theta_{2}^{a}\right)\right)-\mathbf{f}_{\mathscr{F}}\left(\mathbf{\Phi}\left(\theta_{1}^{b}, \theta_{2}^{b}\right)\right)-\sum_{k}\left(\theta_{k}^{a}-\theta_{k}^{b}\right) \nabla_{\mathbf{y}} \mathbf{f}_{\mathscr{F}} \partial_{\theta_{k}} \mathbf{\Phi}(0,0, .)\right\|_{\mathbf{F}_{\delta}^{\infty}} \leq K\left\|\mathbf{f}_{\mathscr{F}}\right\|_{\mathbf{W}^{1, \infty}(\Omega)} R\left\|\theta^{a}-\theta^{b}\right\|_{\Theta_{\delta}^{\infty}} .
$$

In the sequel, we use the compact notations $\mathcal{J}_{\Psi}^{a}=\mathcal{J}_{\mathbf{\Psi}}\left(\theta_{1}^{a}, \theta_{2}^{a}, \mathbf{\Phi}\left(\theta_{1}^{a}, \theta_{2}^{a}, \mathbf{y}\right)\right)$ and $\mathcal{J}_{\Psi}^{b}=\mathcal{J}_{\Psi}\left(\theta_{1}^{b}, \theta_{2}^{b}, \mathbf{\Phi}\left(\theta_{1}^{b}, \theta_{2}^{b}, \mathbf{y}\right)\right)$, and similarly for other functions. We now prove the estimates (C.30)-(C.36). We keep (C.30) for the end.

- Identity (C.31): the proof is immediate.

- Estimate (C.32): we use the decomposition

$$
\begin{aligned}
\mathbf{F}^{2}\left(\theta_{1}^{a}, \theta_{2}^{a}, \mathbf{w}+\mathbf{v}^{a}\right)- & \mathbf{F}^{2}\left(\theta_{1}^{b}, \theta_{2}^{b}, \mathbf{w}+\mathbf{v}^{b}\right)-\mathbf{F}^{2}\left(\theta_{1}^{a}, \theta_{2}^{a}, \mathbf{v}^{a}\right)+\mathbf{F}^{2}\left(\theta_{1}^{b}, \theta_{2}^{b}, \mathbf{v}^{b}\right) \\
& \quad-\sum_{j}\left(\dot{\theta}_{j}^{a}-\dot{\theta}_{j}^{b}\right)\left(\left(\partial_{\theta_{j}} \mathbf{\Phi}(0,0, \mathbf{y}) \cdot \nabla_{\mathbf{y}}\right) \mathbf{w}+\operatorname{cof}\left(\nabla_{\mathbf{y}} \partial_{\theta_{j}} \mathbf{\Phi}(0,0, \mathbf{y})\right)^{T} \mathbf{w}\right) \\
= & -\operatorname{cof}\left(\partial_{t}\left(\mathcal{J}_{\Psi}\left(\theta_{1}^{a}, \theta_{2}^{a}\right)\right) \circ \mathbf{\Phi}\left(\theta_{1}^{a}, \theta_{2}^{a}, \mathbf{y}\right)-\partial_{t}\left(\mathcal{J}_{\Psi}\left(\theta_{1}^{b}, \theta_{2}^{b}\right)\right) \circ \mathbf{\Phi}\left(\theta_{1}^{b}, \theta_{2}^{b}, \mathbf{y}\right)+\sum_{j}\left(\dot{\theta}_{j}^{a}-\dot{\theta}_{j}^{b}\right) \nabla_{\mathbf{y}} \partial_{\theta_{j}} \mathbf{\Phi}(0,0, \mathbf{y})\right)^{T} \mathbf{w} \\
& -\operatorname{cof}\left(\mathcal{J}_{\Psi}^{a}-\mathcal{J}_{\Psi}^{b}\right)^{T} \nabla_{\mathbf{y}} \mathbf{w}\left(\partial_{t} \mathbf{\Psi}\left(\theta_{1}^{a}, \theta_{2}^{a}, .\right)\right) \circ \mathbf{\Phi}\left(\theta_{1}^{a}, \theta_{2}^{a}, \mathbf{y}\right) \\
& -\operatorname{cof}\left(\mathcal{J}_{\Psi}^{b}\right)^{T} \nabla_{\mathbf{y}} \mathbf{w}\left(\partial_{t}\left(\mathbf{\Psi}\left(\theta_{1}^{a}, \theta_{2}^{a}, .\right)\right) \circ \mathbf{\Phi}\left(\theta_{1}^{a}, \theta_{2}^{a}, \mathbf{y}\right)-\partial_{t}\left(\mathbf{\Psi}\left(\theta_{1}^{b}, \theta_{2}^{b}, .\right)\right) \circ \mathbf{\Phi}\left(\theta_{1}^{b}, \theta_{2}^{b}, \mathbf{y}\right)+\sum_{j}\left(\dot{\theta}_{j}^{a}-\dot{\theta}_{j}^{b}\right) \partial_{\theta_{j}} \mathbf{\Phi}(0,0, \mathbf{y})\right) \\
+ & \sum_{j}\left(\dot{\theta}_{j}^{a}-\dot{\theta}_{j}^{b}\right) \operatorname{cof}\left(\mathcal{J}_{\Psi}^{b}-\mathrm{I}\right)^{T} \nabla_{\mathbf{y}} \mathbf{w} \partial_{\theta_{j}} \mathbf{\Phi}(0,0, \mathbf{y}),
\end{aligned}
$$

and the estimates (C.18), (C.26), (C.3), (C.9) and (C.10) yield (C.32).

- Estimate (C.33): we use the decomposition

$$
\begin{aligned}
& \left(\mathbf{F}^{3}\left(\theta_{1}^{a}, \theta_{2}^{a}, \mathbf{w}+\mathbf{v}^{a}\right)-\mathbf{F}^{3}\left(\theta_{1}^{b}, \theta_{2}^{b}, \mathbf{w}+\mathbf{v}^{b}\right)-\mathbf{F}^{3}\left(\theta_{1}^{a}, \theta_{2}^{a}, \mathbf{v}^{a}\right)+\mathbf{F}^{3}\left(\theta_{1}^{b}, \theta_{2}^{b}, \mathbf{v}^{b}\right)\right)_{i}-\nu \sum_{j}\left(\theta_{j}^{a}-\theta_{j}^{b}\right)\left(\mathbf{L}_{\mathbf{F}^{3}}\right)_{i j} \\
& \quad=\left(\mathbf{F}^{3}\left(\theta_{1}^{a}, \theta_{2}^{a}, \mathbf{w}\right)-\mathbf{F}^{3}\left(\theta_{1}^{b}, \theta_{2}^{b}, \mathbf{w}\right)\right)_{i}-\nu \sum_{j}\left(\theta_{j}^{a}-\theta_{j}^{b}\right)\left(\mathbf{L}_{\mathbf{F}^{3}}\right)_{i j} \\
& =A_{1, i}+A_{2, i}+A_{3, i}+A_{4, i},
\end{aligned}
$$

where

$$
\begin{aligned}
A_{1, i}= & \nu \sum_{j, k, \ell, m}\left(\operatorname{cof}\left(\mathcal{J}_{\Psi}^{a}\right)_{k i} \frac{\partial \Psi_{\ell}^{a}}{\partial x_{j}} \frac{\partial \Psi_{m}^{a}}{\partial x_{j}}-\operatorname{cof}\left(\mathcal{J}_{\Psi}^{b}\right)_{k i} \frac{\partial \Psi_{\ell}^{b}}{\partial x_{j}} \frac{\partial \Psi_{m}^{b}}{\partial x_{j}}\right. \\
& \left.+\sum_{n}\left(\theta_{n}^{a}-\theta_{n}^{b}\right)\left(\partial_{y_{j}} \partial_{\theta_{n}} \Phi_{\ell}(0,0, \mathbf{y}) \delta_{k i} \delta_{m j}+\partial_{y_{j}} \partial_{\theta_{n}} \Phi_{m}(0,0, \mathbf{y}) \delta_{k i} \delta_{\ell j}+\operatorname{cof}\left(\partial_{\theta_{n}} \mathcal{J}_{\mathbf{\Phi}}(0,0, \mathbf{y})\right)_{k i} \delta_{m j} \delta_{\ell j}\right)\right) \frac{\partial^{2} w_{k}}{\partial y_{m} \partial y_{\ell},} \\
A_{2, i}= & 2 \nu \sum_{j, k, \ell}\left(\operatorname{cof}\left(\partial_{x_{j}} \mathcal{J}_{\Psi}^{a}\right)_{k i} \frac{\partial \Psi_{\ell}^{a}}{\partial x_{j}}-\operatorname{cof}\left(\partial_{x_{j}} \mathcal{J}_{\Psi}^{b}\right)_{k i} \frac{\partial \Psi_{\ell}^{b}}{\partial x_{j}}-\sum_{n}\left(\theta_{n}^{a}-\theta_{n}^{b}\right) \operatorname{cof}\left(\partial_{x_{j}} \partial_{\theta_{n}} \mathcal{J}_{\Psi}(0,0, \mathbf{y})\right)_{k i} \delta_{\ell j}\right) \frac{\partial w_{k}}{\partial y_{\ell}}, \\
A_{3, i}= & \nu \sum_{j, k, \ell}\left(\operatorname{cof}\left(\mathcal{J}_{\Psi}^{a}\right)_{k i} \frac{\partial^{2} \Psi_{\ell}^{a}}{\partial x_{j}^{2}}-\operatorname{cof}\left(\mathcal{J}_{\Psi}^{b}\right)_{k i} \frac{\partial^{2} \Psi_{\ell}^{b}}{\partial x_{j}^{2}}-\sum_{n}\left(\theta_{n}^{a}-\theta_{n}^{b}\right) \delta_{k i} \frac{\partial^{2}}{\partial x_{j}^{2}} \partial_{\theta_{n}} \Psi_{\ell}(0,0, \mathbf{y})\right) \frac{\partial w_{k}}{\partial y_{\ell}},
\end{aligned}
$$

and

$$
A_{4, i}=\nu \sum_{j, k}\left(\operatorname{cof}\left(\frac{\partial^{2}}{\partial x_{j}^{2}} \mathcal{J}_{\Psi}^{a}\right)_{k i}-\operatorname{cof}\left(\frac{\partial^{2}}{\partial x_{j}^{2}} \mathcal{J}_{\Psi}^{b}\right)_{k i}-\sum_{n}\left(\theta_{n}^{a}-\theta_{n}^{b}\right) \frac{\partial^{2}}{\partial x_{j}^{2}} \partial_{\theta_{n}} \mathcal{J}_{\Psi}(0,0, \mathbf{y})_{k i}\right) w_{k} .
$$

The term $A_{1, i}$ is of the form $\sum_{j, k, \ell, m} a_{i j k \ell m}(\mathbf{y}) \times \frac{\partial^{2} w_{k}}{\partial y_{m} \partial y_{\ell}}$, where

$$
\begin{aligned}
& a_{i j k \ell m}(\mathbf{y})=\left(\operatorname{cof}\left(\mathcal{J}_{\Psi}^{a}\right)_{k i} \frac{\partial \Psi_{\ell}^{a}}{\partial x_{j}} \frac{\partial \Psi_{m}^{a}}{\partial x_{j}}-\operatorname{cof}\left(\mathcal{J}_{\Psi}^{b}\right)_{k i} \frac{\partial \Psi_{\ell}^{b}}{\partial x_{j}} \frac{\partial \Psi_{m}^{b}}{\partial x_{j}}\right. \\
& \left.\quad+\sum_{n}\left(\theta_{n}^{a}-\theta_{n}^{b}\right)\left(\partial_{y_{j}} \partial_{\theta_{n}} \Phi_{\ell}(0,0, \mathbf{y}) \delta_{k i} \delta_{m j}+\partial_{y_{j}} \partial_{\theta_{n}} \Phi_{m}(0,0, \mathbf{y}) \delta_{k i} \delta_{\ell j}+\operatorname{cof}\left(\partial_{\theta_{n}} \mathcal{J}_{\mathbf{\Phi}}(0,0, \mathbf{y})\right)_{k i} \delta_{m j} \delta_{\ell j}\right)\right)
\end{aligned}
$$


Moreover, according to (1.21), $\mathbf{\Psi}\left(\theta_{1}, \theta_{2}, \mathbf{y}\right)=\mathbf{y}$ in $\Omega \backslash \Omega_{\varepsilon}$, then $a_{i j k \ell m}=0$ in $\Omega \backslash \Omega_{\varepsilon}\left(\Omega_{\varepsilon}\right.$ is defined in Lemma 1.4). We then estimate $A_{1, i}$ as

$$
\left\|a_{i j k \ell m} \times \frac{\partial^{2} w_{k}}{\partial y_{m} \partial y_{\ell}}\right\|_{\mathbf{L}^{2}\left(\mathscr{F}_{s}\right)} \leq\left\|\frac{a_{i j k \ell m}}{\prod_{j \in \mathscr{F}_{d, n}} r_{j}^{\beta}}\right\|_{\mathbf{L}^{\infty}\left(\mathscr{F}_{s}\right)}\left\|\frac{\partial^{2} w_{k}}{\partial y_{m} \partial y_{\ell}} \prod_{j \in \mathscr{J}_{d, n}} r_{j}^{\beta}\right\|_{\mathbf{L}^{2}\left(\mathscr{F}_{s}\right)} \leq\left\|a_{i j k \ell m}\right\|_{\mathbf{L}^{\infty}(\Omega)}\left\|\frac{1}{\prod_{j \in \mathscr{F}_{d, n}} r_{j}^{\beta}}\right\|\left\|_{\mathbf{L}^{\infty}\left(\Omega \backslash \Omega_{\varepsilon}\right)}\right\| \mathbf{w} \|_{\mathbf{H}_{\beta}^{2}\left(\mathscr{F}_{s}\right)} .
$$

Moreover, we get the estimate $\left\|a_{i j k \ell m}\right\|_{\mathbf{L}^{\infty}(\Omega)} \leq K R\left\|\theta^{a}-\theta^{b}\right\|_{\Theta_{\delta}^{\infty}}$ by using three times the estimate (C.3). We then use estimates (C.3), (C.4) and (C.19) for $A_{2, i}$ and $A_{3, i}$ and estimate (C.5) for $A_{4, i}$. We obtain (C.33).

- Estimate (C.34): we have the decomposition

$$
\begin{gathered}
\left(\mathbf{F}^{4}\left(\theta_{1}^{a}, \theta_{2}^{a}, \mathbf{w}+\mathbf{v}^{a}\right)-\mathbf{F}^{4}\left(\theta_{1}^{a}, \theta_{2}^{a}, \mathbf{v}^{a}\right)-\mathbf{F}^{4}\left(\theta_{1}^{b}, \theta_{2}^{b}, \mathbf{w}+\mathbf{v}^{b}\right)+\mathbf{F}^{4}\left(\theta_{1}^{b}, \theta_{2}^{b}, \mathbf{v}^{b}\right)+\left(\left(\mathbf{v}^{a}-\mathbf{v}^{b}\right) \cdot \nabla\right) \mathbf{w}\right. \\
\left.+(\mathbf{w} \cdot \nabla)\left(\mathbf{v}^{a}-\mathbf{v}^{b}\right)\right)_{i}-\sum_{n}\left(\theta_{n}^{a}-\theta_{n}^{b}\right)\left(\mathbf{L}_{\mathbf{F}^{4}}\right)_{i, n} \\
=B_{1, i}+B_{2, i}+B_{3, i}+B_{4, i}+B_{5, i}+B_{6, i}
\end{gathered}
$$

where

$$
\begin{aligned}
B_{1, i} & =-\sum_{j, k, \ell}\left(\operatorname{cof}\left(\mathcal{J}_{\Psi}^{a}\right)_{k j} \operatorname{cof}\left(\partial_{x_{j}} \mathcal{J}_{\Psi}^{a}\right)_{\ell i}-\operatorname{cof}\left(\mathcal{J}_{\Psi}^{b}\right)_{k j} \operatorname{cof}\left(\partial_{x_{j}} \mathcal{J}_{\Psi}^{b}\right)_{\ell i}-\sum_{n}\left(\theta_{n}^{a}-\theta_{n}^{b}\right) \operatorname{cof}\left(\partial_{x_{j}} \partial_{\theta_{n}} \mathcal{J}_{\Psi}(0,0, \mathbf{y})\right)_{\ell i} \delta_{k j}\right) w_{k} w_{\ell}, \\
B_{2, i} & =-\sum_{j, k, \ell}\left(\operatorname{cof}\left(\mathcal{J}_{\Psi}^{a}\right)_{k j} \operatorname{cof}\left(\partial_{x_{j}} \mathcal{J}_{\Psi}^{a}\right)_{\ell i}-\operatorname{cof}\left(\mathcal{J}_{\Psi}^{b}\right)_{k j} \operatorname{cof}\left(\partial_{x_{j}} \mathcal{J}_{\Psi}^{b}\right)_{\ell i}\right)\left(w_{k} v_{\ell}^{a}+v_{k}^{a} w_{\ell}\right), \\
B_{3, i} & =-\sum_{j, k, \ell} \operatorname{cof}\left(\mathcal{J}_{\Psi}^{b}\right)_{k j} \operatorname{cof}\left(\partial_{x_{j}} \mathcal{J}_{\Psi}^{b}\right)_{\ell i}\left(w_{k}\left(v_{\ell}^{a}-v_{\ell}^{b}\right)+\left(v_{k}^{a}-v_{k}^{b}\right) w_{\ell}\right), \\
B_{4, i} & =-\sum_{k, \ell}\left(\operatorname{det}\left(\mathcal{J}_{\Psi}^{a}\right)^{2} \frac{\partial \Phi_{i}^{a}}{\partial y_{\ell}}-\operatorname{det}\left(\mathcal{J}_{\Psi}^{b}\right)^{2} \frac{\partial \Phi_{i}^{b}}{\partial y_{\ell}}+\sum_{n}\left(\theta_{n}^{a}-\theta_{n}^{b}\right)\left(2 \operatorname{Tr}\left(\partial_{\theta_{n}} \mathcal{J}_{\mathbf{\Phi}}(0,0, \mathbf{y})\right) \delta_{i \ell}-\partial_{\theta_{n}} \mathcal{J}_{\boldsymbol{\Phi}}(0,0, \mathbf{y})_{i \ell}\right)\right) w_{k} \frac{\partial w_{\ell}}{\partial y_{k}}, \\
B_{5, i}= & -\sum_{k, \ell}\left(\operatorname{det}\left(\mathcal{J}_{\Psi}^{a}\right)^{2} \frac{\partial \Phi_{i}^{a}}{\partial y_{\ell}}-\operatorname{det}\left(\mathcal{J}_{\Psi}^{b}\right)^{2} \frac{\partial \Phi_{i}^{b}}{\partial y_{\ell}}\right)\left(\frac{\partial w_{\ell}}{\partial y_{\ell}} v_{k}^{a}+\frac{\partial v_{\ell}^{a}}{\partial y_{\ell}} w_{k}\right),
\end{aligned}
$$

and$$
B_{6, i}=-\sum_{k, \ell}\left(\operatorname{det}\left(\mathcal{J}_{\Psi}^{b}\right)^{2} \frac{\partial \Phi_{i}^{b}}{\partial y_{\ell}}-\delta_{i \ell}\right)\left(\frac{\partial w_{\ell}}{\partial y_{k}}\left(v_{k}^{a}-v_{k}^{b}\right)+\left(\frac{\partial v_{\ell}^{a}}{\partial y_{k}}-\frac{\partial v_{\ell}^{b}}{\partial y_{k}}\right) w_{k}\right) .
$$

Now, we use

- estimates (C.3), (C.4), (C.18) and (C.19) for $B_{1, i}$,

- estimates (C.3), (C.4) for $B_{2, i}$,

- estimates (C.18) and (C.19) for $B_{3, i}$,

- estimates (C.2), (C.7), (C.17) and (C.18) for $B_{4, i}$ and $B_{5, i}$,

- estimates (C.17) and (C.18) for $B_{6, i}$.

We get (C.34).

- Estimate (C.35): we have

$\mathbf{F}^{5}\left(\theta_{1}^{a}, \theta_{2}^{a}, p_{\mathbf{w}}+q^{a}\right)-\mathbf{F}^{5}\left(\theta_{1}^{a}, \theta_{2}^{a}, q^{a}\right)-\mathbf{F}^{5}\left(\theta_{1}^{b}, \theta_{2}^{b}, p_{\mathbf{w}}+q^{b}\right)+\mathbf{F}^{5}\left(\theta_{1}^{b}, \theta_{2}^{b}, q^{b}\right)-\sum_{n}\left(\theta_{n}^{a}-\theta_{n}^{b}\right)\left(\nabla_{\mathbf{y}} \partial_{\theta_{n}} \mathbf{\Phi}(0,0, \mathbf{y})\right)^{T} \nabla_{\mathbf{y}} p_{\mathbf{w}}$

$$
\begin{aligned}
& =\mathbf{F}^{5}\left(\theta_{1}^{a}, \theta_{2}^{a}, p_{\mathbf{w}}\right)-\mathbf{F}^{5}\left(\theta_{1}^{b}, \theta_{2}^{b}, p_{\mathbf{w}}\right)-\sum_{n}\left(\theta_{n}^{a}-\theta_{n}^{b}\right)\left(\nabla_{\mathbf{y}} \partial_{\theta_{n}} \mathbf{\Phi}(0,0, \mathbf{y})\right)^{T} \nabla_{\mathbf{y}} p_{\mathbf{w}} \\
& =-\left(\mathcal{J}_{\Psi}^{a}-\mathcal{J}_{\mathbf{\Psi}}^{b}+\sum_{n}\left(\theta_{n}^{a}-\theta_{n}^{b}\right) \nabla_{\mathbf{y}} \partial_{\theta_{n}} \mathbf{\Phi}(0,0, \mathbf{y})\right)^{T} \nabla_{\mathbf{y}} p_{\mathbf{w}},
\end{aligned}
$$

and estimate (C.3) gives (C.35).

- Estimate (C.36): it is a consequence of (C.1).

- Estimate (C.30): we have

$$
\begin{gathered}
\left(\mathbf{S}\left(\theta_{1}^{a}, \theta_{2}^{a}, \mathbf{w}+\mathbf{v}^{a}, p_{\mathbf{w}}+q^{a}\right)-\mathbf{S}\left(\theta_{1}^{a}, \theta_{2}^{a}, \mathbf{v}^{a}, q^{a}\right)-\mathbf{S}\left(\theta_{1}^{b}, \theta_{2}^{b}, \mathbf{w}+\mathbf{v}^{b}, p_{\mathbf{w}}+q^{b}\right)+\mathbf{S}\left(\theta_{1}^{b}, \theta_{2}^{b}, \mathbf{v}^{b}, q^{b}\right)-\mathbf{L}_{\mathbf{S}}\left(\theta_{1}^{a}-\theta_{1}^{b}, \theta_{2}^{a}-\theta_{2}^{b}\right)\right)_{j} \\
=D_{1, j}+D_{2, j}+D_{3, j}+D_{4, j},
\end{gathered}
$$


where

$$
\begin{aligned}
& D_{1, j}=\int_{\partial S_{s}}\left(\left|\mathcal{J}_{\boldsymbol{\Phi}}^{a} \mathbf{t}_{s}\right|-\left|\mathcal{J}_{\boldsymbol{\Phi}}^{b} \mathbf{t}_{s}\right|\right)\left(p_{\mathbf{w}} \mathrm{I}-\nu\left(\mathcal{G}^{a}+\left(\mathcal{G}^{a}\right)^{T}\right)\right)\left(\mathbf{n}_{\theta_{1}, \theta_{2}}^{a} \circ \boldsymbol{\Phi}^{a}\right) \cdot \partial_{\theta_{j}} \boldsymbol{\Phi}^{a} \\
&+\sum_{n}\left(\theta_{n}^{a}-\theta_{n}^{b}\right) \int_{\partial S_{s}}\left(\left(\nabla_{\mathbf{y}} \partial_{\theta_{n}} \mathbf{\Phi}\left(0,0, \gamma_{y}\right)\right) \mathbf{t}_{s} \cdot \mathbf{t}_{s}\right) \sigma_{F}\left(\mathbf{w}, p_{\mathbf{w}}\right) \mathbf{n}_{s} \cdot \partial_{\theta_{j}} \mathbf{\Phi}\left(0,0, \gamma_{y}\right), \\
& D_{2, j}=-\nu \int_{\partial S_{s}}\left|\mathcal{J}_{\mathbf{\Phi}}^{b} \mathbf{t}_{s}\right|\left(\mathcal{G}^{a}+\left(\mathcal{G}^{a}\right)^{T}-\mathcal{G}^{b}-\left(\mathcal{G}^{b}\right)^{T}\right)\left(\mathbf{n}_{\theta_{1}, \theta_{2}}^{a} \circ \boldsymbol{\Phi}^{a}\right) \cdot \partial_{\theta_{j}} \boldsymbol{\Phi}^{a}+\nu \sum_{k, \ell, n}\left(\theta_{n}^{a}-\theta_{n}^{b}\right) \int_{\partial S_{s}}\left(\left(\mathbf{L}_{\mathcal{G}}\right)_{k \ell n}+\left(\mathbf{L}_{\mathcal{G}}\right)_{\ell k n}\right)\left(\mathbf{n}_{s}\right)_{k} \partial_{\theta_{j}} \Phi_{\ell}\left(0,0, \gamma_{y}\right), \\
& D_{3, j}=\int_{\partial S_{s}}\left|\mathcal{J}_{\mathbf{\Phi}}^{b} \mathbf{t}_{s}\right|\left(p_{\mathbf{w}} \mathrm{I}-\nu\left(\mathcal{G}^{b}+\left(\mathcal{G}^{b}\right)^{T}\right)\right)\left(\mathbf{n}_{\theta_{1}, \theta_{2}}^{a} \circ \boldsymbol{\Phi}^{a}-\mathbf{n}_{\theta_{1}, \theta_{2}}^{b} \circ \boldsymbol{\Phi}^{b}\right) \cdot \partial_{\theta_{j}} \boldsymbol{\Phi}^{a} \\
&+\sum_{k, \ell, n}\left(\theta_{n}^{a}-\theta_{n}^{b}\right) \int_{\partial S_{s}} \sigma_{F}\left(\mathbf{w}, p_{\mathbf{w}}\right)_{\ell k}\left(\mathbf{L}_{\mathbf{n}_{\theta_{1}, \theta_{2}}}\right)_{k n} \partial_{\theta_{j}} \Phi_{\ell}\left(0,0, \gamma_{y}\right),
\end{aligned}
$$

and

$$
\begin{aligned}
D_{4, j}=\int_{\partial S_{s}}\left|\mathcal{J}_{\boldsymbol{\Phi}}^{b} \mathbf{t}_{s}\right|\left(p_{\mathbf{w}} \mathrm{I}-\nu\left(\mathcal{G}^{b}+\left(\mathcal{G}^{b}\right)^{T}\right)\right)\left(\mathbf{n}_{\theta_{1}, \theta_{2}}^{b} \circ \boldsymbol{\Phi}^{b}\right) \cdot\left(\partial_{\theta_{j}} \boldsymbol{\Phi}^{a}-\partial_{\theta_{j}} \boldsymbol{\Phi}^{b}\right) \\
\quad+\sum_{n}\left(\theta_{n}^{a}-\theta_{n}^{b}\right) \int_{\partial S_{s}} \sigma_{F}\left(\mathbf{w}, p_{\mathbf{w}}\right) \mathbf{n}_{s} \cdot \partial_{\theta_{n} \theta_{j}} \mathbf{\Phi}\left(0,0, \gamma_{y}\right) .
\end{aligned}
$$

We use

- estimates (C.8), (C.28), (C.22) and (C.24) for $D_{1, j}$,

- estimates (C.23), (C.16), (C.22) and (C.24) for $D_{2, j}$,

- estimates (C.23), (C.28), (C.6) and (C.24) for $D_{3, j}$,

- estimates (C.23), (C.28), (C.22) and (C.12) for $D_{4, j}$.

Combining these estimates yields (C.30).

\section{References}

[1] M. Badra. Feedback stabilization of the 2-D and 3-D Navier-Stokes equations based on an extended system. ESAIM Control Optim. Calc. Var., 15(4):934-968, 2009.

[2] M. Badra and T. Takahashi. Stabilization of parabolic nonlinear systems with finite dimensional feedback or dynamical controllers: application to the Navier-Stokes system. SIAM J. Control Optim., 49(2):420-463, 2011.

[3] M. Badra and T. Takahashi. Feedback stabilization of a fluid-rigid body interaction system. Adv. Differential Equations, 19(11-12):1137-1184, 2014.

[4] M. Badra and T. Takahashi. On the Fattorini criterion for approximate controllability and stabilizability of parabolic systems. ESAIM Control Optim. Calc. Var., 20(3):924-956, 2014.

[5] M. Badra and T. Takahashi. Feedback boundary stabilization of 2D fluid-structure interaction systems. Discrete Contin. Dyn. Syst., 37(5):2315-2373, 2017.

[6] A. Bensoussan, G. Da Prato, M. C. Delfour, and S. K. Mitter. Representation and control of infinite dimensional systems. Systems \& Control: Foundations \& Applications. Birkhäuser Boston, Inc., Boston, MA, second edition, 2007.

[7] N. Cîndea, S. Micu, I. Rovenţa, and M. Tucsnak. Particle supported control of a fluid-particle system. J. Math. Pures Appl. (9), 104(2):311-353, 2015.

[8] S. Court. Stabilization of a fluid-solid system, by the deformation of the self-propelled solid. Part I: The linearized system. Evol. Equ. Control Theory, 3(1):59-82, 2014.

[9] S. Court. Stabilization of a fluid-solid system, by the deformation of the self-propelled solid. Part II: The nonlinear system. Evol. Equ. Control Theory, 3(1):83-118, 2014.

[10] R. F. Curtain and H. Zwart. An introduction to infinite-dimensional linear systems theory, volume 21 of Texts in Applied Mathematics. Springer-Verlag, New York, 1995. 
[11] G. Delay. Existence of strong solutions to a fluid-structure system with a structure given by a finite number of parameters. Submitted, available on https://www.math.univ-toulouse.fr/ gdelay/.

[12] C. Fabre and G. Lebeau. Prolongement unique des solutions de l'equation de Stokes. Comm. Partial Differential Equations, 21(3-4):573-596, 1996.

[13] A. V. Fursikov. Stabilizability of a quasilinear parabolic equation by means of boundary feedback control. Mat. Sb., 192(4):115-160, 2001.

[14] A. V. Fursikov and R. Rannacher. Optimal Neumann control for the two-dimensional steady-state NavierStokes equations. In New directions in mathematical fluid mechanics, Adv. Math. Fluid Mech., pages 193-221. Birkhäuser Verlag, Basel, 2010.

[15] W. R. Graham, J. Peraire, and K. Y. Tang. Optimal control of vortex shedding using low-order models. I. Open-loop model development. Internat. J. Numer. Methods Engrg., 44(7):945-972, 1999.

[16] W.R. Graham, J. Peraire, and K.Y. Tang. Optimal control of vortex shedding using low-order models. II. Model-based control. Internat. J. Numer. Methods Engrg., 44(7):973-990, 1999.

[17] P. Grisvard. Elliptic problems in nonsmooth domains, volume 69 of Classics in Applied Mathematics. Society for Industrial and Applied Mathematics (SIAM), Philadelphia, PA, 2011.

[18] D. Kholodar and E. Dowell. The influence of a nonzero angle of attack and gust loads on the nonlinear response of a typical airfoil section with a control surface freeplay. Int. Journal of Nonlinear Sc. and Num. Simulation, 1(3):153-166, 2000.

[19] J.-L. Lions and E. Magenes. Problèmes aux limites non homogènes et applications. Vol. 1. Travaux et Recherches Mathématiques, No. 17. Dunod, Paris, 1968.

[20] V. Maz'ya and J. Rossmann. Elliptic equations in polyhedral domains, volume 162 of Mathematical Surveys and Monographs. American Mathematical Society, Providence, RI, 2010.

[21] K. K. Nagarajan, L. Cordier, and C. Airiau. Development and application of a reduced order model for the control of self-sustained instabilities in cavity flows. Commun. Comput. Phys., 14(1):186-218, 2013.

[22] M. Ndiaye. Stabilisation et simulation de modèles d'interaction fluide-structure. PhD thesis, Université de Toulouse, 2016.

[23] P. A. Nguyen and J.-P. Raymond. Boundary stabilization of the Navier-Stokes equations in the case of mixed boundary conditions. SIAM J. Control Optim., 53(5):3006-3039, 2015.

[24] A. Pazy. Semigroups of linear operators and applications to partial differential equations, volume 44 of Applied Mathematical Sciences. Springer-Verlag, New York, 1983.

[25] J.-P. Raymond. Feedback boundary stabilization of the two-dimensional Navier-Stokes equations. SIAM J. Control Optim., 45(3):790-828, 2006.

[26] J.-P. Raymond. Feedback boundary stabilization of the three-dimensional incompressible Navier-Stokes equations. J. Math. Pures Appl. (9), 87(6):627-669, 2007.

[27] J.-P. Raymond. Feedback stabilization of a fluid-structure model. SIAM J. Control Optim., 48(8):53985443, 2010.

[28] R. Semaan, P. Kumar, M. Burnazzi, G. Tissot, L. Cordier, and B. R. Noack. Reduced-order modelling of the flow around a high-lift configuration with unsteady Coanda blowing. J. Fluid Mech., 800:72-110, 2016.

[29] T. Takahashi, M. Tucsnak, and G. Weiss. Stabilization of a fluid-rigid body system. J. Differential Equations, 259(11):6459-6493, 2015.

[30] M. Tucsnak and G. Weiss. Observation and control for operator semigroups. Birkhäuser Advanced Texts: Basler Lehrbücher. [Birkhäuser Advanced Texts: Basel Textbooks]. Birkhäuser Verlag, Basel, 2009. 